\title{
Rh(III)-Catalyzed Three-Component Syn-Carboamination of Alkenes Using Arylboronic Acids and Dioxazolones
}

Sumin Lee, Tomislav Rovis

Submitted date: $31 / 03 / 2021 \cdot$ Posted date: 01/04/2021

Licence: CC BY-NC-ND 4.0

Citation information: Lee, Sumin; Rovis, Tomislav (2021): Rh(III)-Catalyzed Three-Component Syn-Carboamination of Alkenes Using Arylboronic Acids and Dioxazolones. ChemRxiv. Preprint. https://doi.org/10.26434/chemrxiv.14347277.v1

Herein we report a Rh(III)-catalyzed three-component carboamination of alkenes from readily available aryl boronic acids as a carbon source and dioxazolones as nitrogen electrophiles. This protocol provides facile access to valuable amine products including a-amino acid derivatives in good yield and excellent regioselectivity without the need for a directing functionality. A series of experiments suggest a mechanism in which the $\mathrm{Rh}$ (III) catalyst undergoes transmetalation with the aryl boronic acid followed by turnover limiting, alkene migratory insertion into the Rh(III)-aryl bond. Subsequently, fast Rh-nitrene formation provides the syn-carboamination product selectively after reductive elimination and proto-demetalation. Importantly, the protocol provides 3-component coupling products in preference to a variety of 2-component undesired by-products.

File list (2)

article_ChemRxiv.pdf (1.36 MiB) view on ChemRxiv • download file

SI_ChemRxiv.pdf (8.32 MiB) view on ChemRxiv - download file 


\title{
Rh(III)-Catalyzed Three-Component Syn-Carboamination of Alkenes Using Arylboronic Acids and Dioxazolones
}

Sumin Lee and Tomislav Rovis*

Department of Chemistry, Columbia University, New York, New York, 10027, United States

Contact information: tr2504@columbia.edu

\begin{abstract}
Herein we report a $\mathrm{Rh}(\mathrm{III})$-catalyzed three-component carboamination of alkenes from readily available aryl boronic acids as a carbon source and dioxazolones as nitrogen electrophiles. This protocol provides facile access to valuable amine products including $\alpha$-amino acid derivatives in good yield and excellent regioselectivity without the need for a directing functionality. A series of experiments suggest a mechanism in which the Rh(III) catalyst undergoes transmetalation with the aryl boronic acid followed by turnover limiting, alkene migratory insertion into the Rh(III)-aryl bond. Subsequently, fast Rh-nitrene formation provides the syn-carboamination product selectively after reductive elimination and proto-demetalation. Importantly, the protocol provides 3-component coupling products in preference to a variety of 2-component undesired by-products.
\end{abstract}

The simultaneous installation of two functional groups across the ubiquitous alkene double bond in a stereoselective manner is a powerful transformation in organic synthesis, ${ }^{1,2,3,4,5,6}$ and provides an efficient way of rapidly increasing molecular complexity from readily available starting materials. Among various potential difunctionalizations, the carboamination of alkenes offers direct access to valuable and pharmaceutically important amine products by forming both $\mathrm{C}-\mathrm{C}$ and $\mathrm{C}-\mathrm{N}$ bonds in a single step. ${ }^{7,8}$ A handful of powerful annulations ${ }^{9,10}$ or intramolecular carboaminations $\mathrm{s}^{11,12,13}$ have been developed, which are currently limited to the synthesis of cyclic products. In 2015, 
we described a Rh(III)-catalyzed stereospecific, syn-carboamination of activated alkenes using $N$-enoxyphthalimide as both carbon and nitrogen source of the reaction to furnish acyclic amine products (Scheme 1a). ${ }^{14}$ Subsequently, Glorius reported the carboamination of acrylates initiated by $\mathrm{Cp}^{*} \mathrm{Co}^{\mathrm{III}}$-catalyzed $\mathrm{C}^{\mathrm{sp} 2}-\mathrm{H}$ activation of $N-$ phenoxyamides (Scheme 1b), ${ }^{15,16}$ followed more recently by Cramer's demonstration of an asymmetric version of this reaction. ${ }^{17}$ Recently, Ellman reported the synthesis of $\alpha$-branched amine through the Rh(III)-catalyzed threecomponent 1,1-carboamination of terminal alkenes that also initiated by directing group assisted C-H activation. ${ }^{18}$ Other significant contributions towards the linear carboamination of alkenes have been made utilizing Pd or $\mathrm{Ni}$ catalysis with olefins bearing covalently linked aminoquinoline directing groups, ${ }^{19,20}$ as well as through single electron pathways involving nitrogen or carbon centered radicals..$^{2 b, 21,22,23}$

Despite recent progress in the field, the current state-of-the-art in carboamination involves additional steps to install and remove the often undesired directing functionality, an inherent loss in stereocontrol imparted through open shell intermediates, or synthetically taxing substrates that limit the chemical space available to their practical application. In searching for a more general solution to this problem, we became interested in developing an intermolecular 3component carboamination that uses readily accessible carbon and nitrogen sources. Such a modular approach would enable the rapid synthesis of a diverse library of functionalized amine products by simply switching coupling partners. As a reaction design to achieve this goal, we envisioned that a carbon nucleophile coordinates to $\mathrm{Cp} * \mathrm{Rh}(\mathrm{III})$ complex after transmetalation or ligand displacement then undergoes highly regioselective migratory insertion with activated alkenes in the absence of directing group. Subsequent reaction with nitrene precursors forms Rh-nitrene intermediates which will undergo reductive elimination to form a $\mathrm{C}-\mathrm{N}$ bond and deliver desired carboamination products (Scheme 1c). The challenge is that there are several undesired side product pathways as the reaction becomes a multi-component system. For example, after transmetalation, it can undergo dimerization of carbon source or direct $\mathrm{C}-\mathrm{N}$ coupling if subsequent alkene migratory insertion is slow. Also, after migratory insertion of Rh-carbon bond into the alkenes, undesired $\beta$-hydride elimination or protodemetalation will result in Heck-type and hydroarylation products. Last, hydroamination side product also can be formed if the nitrogen source of the reaction directly reacts with the alkene coupling partner. 


\section{Scheme 1. Intermolecular carboamination of alkenes.}

Previous work : carboamination employing a directing group

(a) ref. 14

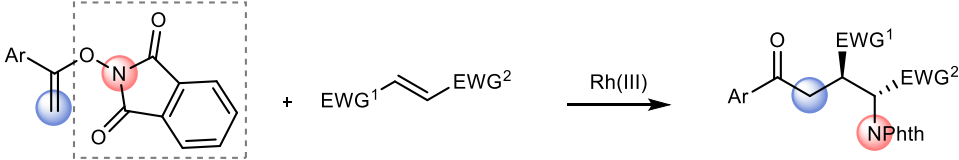

(b) ref. 15
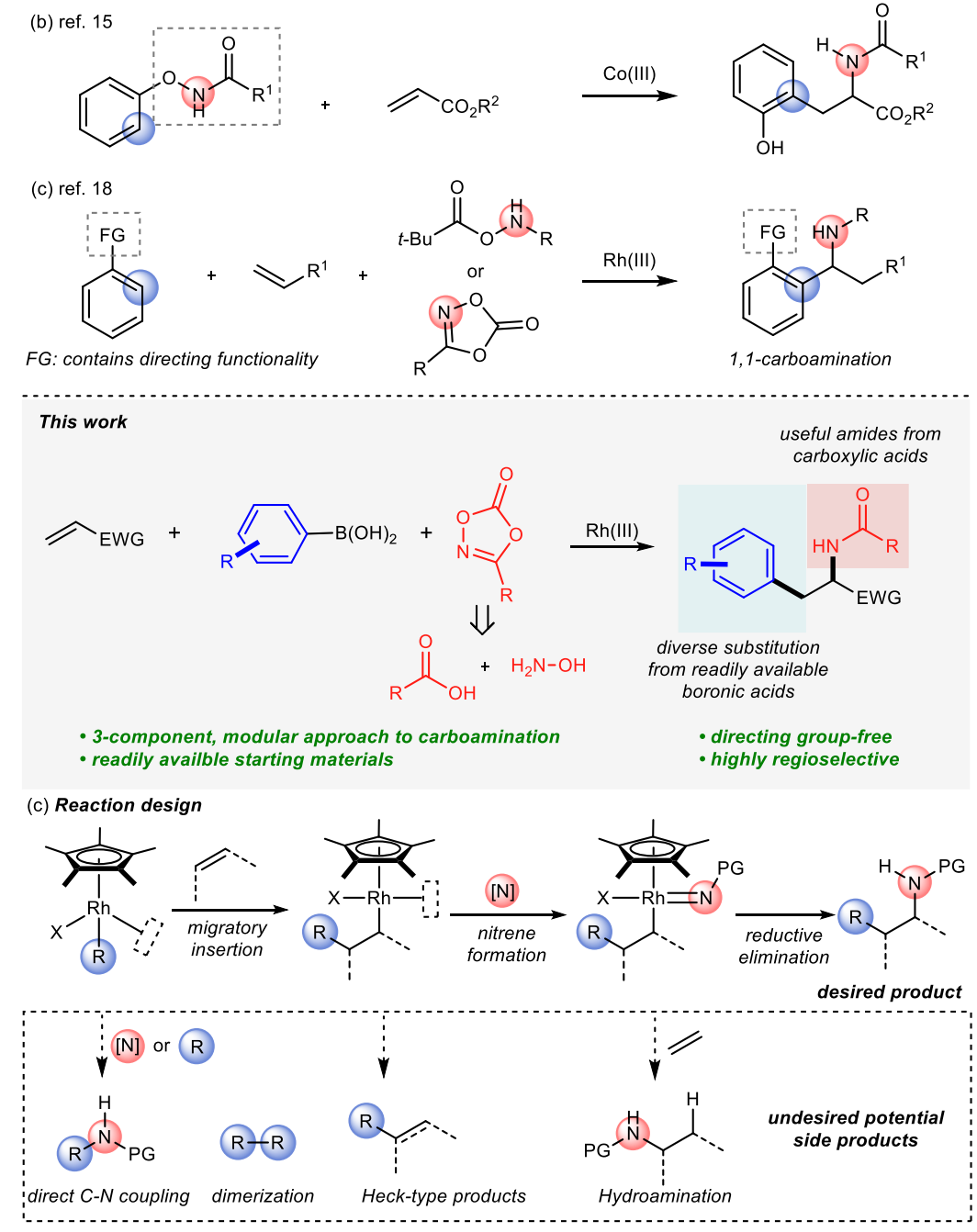

Challenges: achieve 3-component coupling in preference to myriad 2-component pathways

\section{Results}

Reaction development. With this hypothesis in mind, we initiated a systematic investigation of carbon nucleophiles such as organoboron and organostannanes, with nitrene precursors such as azides, hydroxamates, and dioxazolones, under various reaction conditions. An initial hit was identified when we combined phenylboronic acid (1a), benzyl acrylate (2a), and 3-methyl-1,4,2-dioxazol-5-one (3a) with $\left[\mathrm{Cp}^{*} \mathrm{RhCl}_{2}\right]_{2}$ as a catalyst in methanol at room 
temperature delivering the desired carboamination product (4a) in $8 \%$ yield, with the mass balance comprised of Heck-type, hydroamination, and direct C-N coupling products. Inspired by a large library of commercially available boronic acids and readily accessible dioxazolones which can be easily prepared from carboxylic acids,${ }^{24}$ we decided to optimize the reaction and gratifyingly, achieved a 77\% yield of the desired carboamination product (4a) (Table 1 , entry 1 , see supporting information for details).

Other nitrene precursors ${ }^{10,25,26,27}$ that are frequently used for amination chemistry with Cp* group 9 catalysis are completely ineffective for this chemistry. In the cases of Ts- $\mathrm{N}_{3}$, Ts-NH-OPiv, 5,5-dimethyl-1,4,2-dioxazole, and 1,4,2-dioxazol-5-thione, no conversion of starting materials is observed. When $N$-(pivaloyloxy)amides are used as nitrogen source of the reaction, direct C-N coupling product was observed as major side product and $N$ (tetrafluorophenoxy)amides give Heck-type and hydroarylation side products in high yield. (entry 2, see supporting information for details, Table S1, page 6). Also, the choice of the catalyst is important since cationic Rh(III) catalysts such as $\left[\mathrm{Cp}^{*} \mathrm{Rh}(\mathrm{MeCN})_{3}\right]\left(\mathrm{SbF}_{6}\right)_{2}$ (entry 3) or an in situ generated cationic $\mathrm{Rh}(\mathrm{III})$ complex does not lead to the desired product. Several $\mathrm{Rh}(\mathrm{I})$ catalysts such as $\left[\mathrm{Rh}(\mathrm{COD})_{2} \mathrm{Cl}\right]_{2}$ (entry 4) that are commonly used for conjugate addition chemistry with arylboronic acids ${ }^{28,29,30}$ or other $\mathrm{Cp} *$ group 9 catalysts such as $\left[\mathrm{Cp}^{*} \mathrm{CoCl}_{2}\right]_{2}$ or $\left[\mathrm{Cp}^{*} \mathrm{IrCl}_{2}\right]_{2}$ failed to deliver the carboamination products. Screening of base additives shows that bicarbonate, carbonate, or fluoride generally gives a higher reaction yield compared to acetates, and $\mathrm{NaHCO}_{3}$ was selected as the optimal additive. When acetates are used as additive, the reaction yield is significantly decreased and Heck-type product becomes major product of the reaction in low yield. One possible explanation is that acetates act as bidentate ligand and prevent coordination of dioxazolone (entry 5). The reaction proceeds smoothly at room temperature and tends to give a lower yield at a higher temperature, as shown with the $42 \%$ yield obtained when the reaction was conducted at $60{ }^{\circ} \mathrm{C}$ (entry 6). When both alkene (2a) and dioxazolone (3a) are used as the limiting reagent of the reaction, the reaction gives synthetically useful a 64\% yield (entry 7), and a 77\% yield was achieved when $\mathbf{1 a}$ (2.5 equiv), 2a (3 equiv), and 3a (1 equiv) was used for the reaction (See supporting information for details, Table S4, page 9). Lastly, the reaction can be performed on a $1.0 \mathrm{mmol}$ scale with $2.5 \mathrm{~mol} \%$ catalyst loading without loss of the product yield (entry 8). 
Table 1. Reaction Optimization

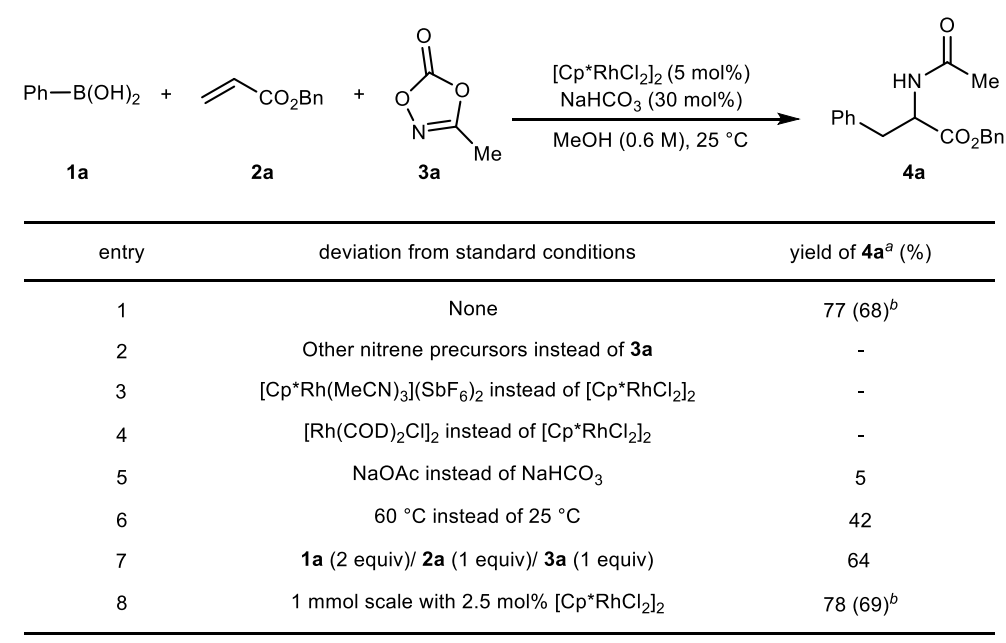

Reactions were conducted on a $0.1 \mathrm{mmol}$ scale using 1a (2.5 equiv), 2a (3.0 equiv), and 3a (1 equiv). ${ }^{a}$ Determined by analysis of ${ }^{1} \mathrm{H}$ NMR of the unpurified reaction mixture. ${ }^{b}$ Isolated yield.

Reaction scope. Having optimized the reaction conditions, we next sought to explore the scope of the transformation (Scheme 2). The reaction with aryl boronic acids containing a variety of functional groups at the para position of phenyl ring proceeds smoothly, delivering products with $-\mathrm{CF}_{3}(\mathbf{4 b}),-\mathrm{F}(\mathbf{4 c}),-\mathrm{Br}(\mathbf{4 d}),-\mathrm{OMe}(\mathbf{4 e})$, hydroxy (4f), silyl ether (4g), alkene (4h), alkyl (4i), nitrile (4j), and aldehyde (4k) functional groups in good yield. Aryl boronic acids with a substituent in the meta position (4l), having multiple substituents (4o, 4p), naphthyl boronic acid (4q), and 3,4-methylenedioxyphenylboronic acid (4r) also work well in this reaction. In the case of vinyl boronic acids such as 1-penten-1-ylboronic acid or alkyl boronic acids, the reactions are unsuccessful. (See supporting information for limitations, page 44).

For alkene substrate scope, the reaction with monosubstituted acrylates gives corresponding phenylalanine derivatives (5d-5d) in good yield. Acrylonitrile (2e) also works well in this reaction, providing a potential for further functional group derivatization. Secondary acrylamides are also good alkene coupling partners, delivering carboamination products $(\mathbf{5 f}-\mathbf{5 h})$ containing two amide bonds.

The reaction with bridged bicyclic alkenes such as norbornene (2i), oxabenzonorbornadiene (2j), and aza-

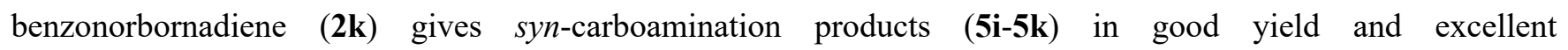


diastereoselectivity. In the case of the reactions with cyclopropenes (21-2m), the reaction occurs cis to the methyl group, presumably because of a smaller steric demand during the alkene migratory insertion step, giving

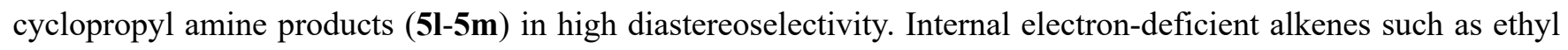
crotonate, ethyl cinnamate, or cyclopentenone and less-strained cyclic alkenes such as cyclohexene or 3,4dihydropyran do not lead to the product formation and direct $N$-phenylation (9) products are observed as a major side product of the reaction in high yield presumably due to the slow alkene migratory insertion (see supporting information for details, page 44). Lastly, a variety of functional groups can be installed at the amide side chain using dioxazolones with different substituents at the 3 position (3b-3h) prepared from corresponding carboxylic acids. The reactions with dioxazolone 3d or $\mathbf{3 f}$ containing allylic or benzylic $\mathrm{C}-\mathrm{H}$ bonds give the carboamination products selectively $(\mathbf{6 d}, \mathbf{6 f})$. These are noteworthy as they have been used for intramolecular C-H amination chemistry. ${ }^{31}$ The carboamination occurs intermolecularly in preference to an intramolecular $\mathrm{C}-\mathrm{H}$ insertion suggesting a mechanistic dichotomy with Chang's Ir-based system.

Mechanistic investigation. We next designed a series of experiments to interrogate the mechanism of the reaction. First, we conducted the reaction under standard conditions, but without phenylboronic acid (Scheme 3a). As a result, neither $N$-acyl aziridine nor aziridine ring-opening products ${ }^{32}$ are observed after the reaction. This strongly indicates that the $\mathrm{Rh}(\mathrm{III})$-catalyzed alkene aziridination and subsequent ring-opening with phenylboronic acid is unlikely as the mechanism of the reaction. During the optimization study, we observed Heck-type (7) and hydroarylation products $(\mathbf{8})$ as side products of the reaction, presumably formed through $\beta$-hydride elimination and proto-demetallation after the alkene migratory insertion. To determine whether these side products give the desired carboamination product (4), both benzyl cinnamate (7) and benzyl 3-phenylpropanoate (8) were subjected to the reaction using 1-naphthyl boronic acid (2q) as the carbon source of the reaction (Scheme 3b). While both reactions give the desired product (4q) from 1-naphthyl boronic acid (2q) in good yield, carboamination products (4a) from these side products ( 7 or $\mathbf{8}$ ) are not observed. These experiments suggest $\mathbf{7}$ and $\mathbf{8}$ are off-cycle side products that do not re-enter the catalytic cycle. 
Scheme 2. Reaction Scope

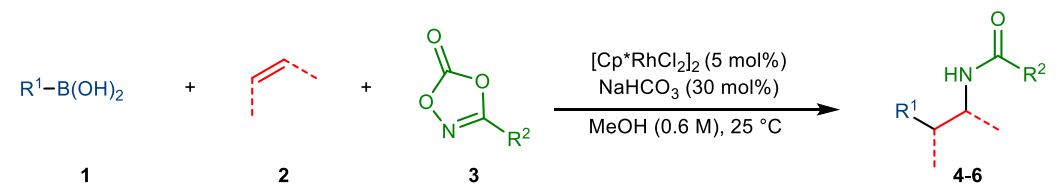

Boronic acid scope<smiles>COc1ccc(CC(NC(C)=O)C(=O)OCc2ccc(C(=O)OCc3ccccc3)cc2)cc1</smiles>

4a, $68 \%$

4b, $62 \%$

4c, $60 \%$

4d, $65 \%$

4e, $60 \%$

4f, $70 \%$<smiles>C=Cc1ccc(CC(NC(=O)C(C)(C)C)C(=O)c2ccccc2)cc1</smiles>

4g, $66 \%$

4h, $55 \%$

4i, $55 \%$<smiles>CC(=O)NC(Cc1ccc(C#N)cc1)C(=O)OCc1ccccc1</smiles>

4j, $51 \%$<smiles>COC(=O)c1cccc(CC(NC(C)=O)C(=O)OCc2ccccc2)c1</smiles>

4k, $64 \%$

$4 \mathrm{I}, 65 \%$<smiles>CC(=O)N[C@@H](Cc1ccccc1F)[18O][Na]</smiles>

$4 \mathrm{~m}, 74 \%$<smiles>CCOC(Cc1ccccc1)NC(C)=O</smiles>

5b, $68 \%$<smiles>CC(=O)NC(Cc1ccccc1)C(=O)NCC1CCN(C(C)(C)C)CC1</smiles>

5h, $74 \%$<smiles>CC(=O)N[C@H](Cc1cc(C(C)=O)cc(C(C)(F)F)c1)Cc1ccccc1Cl</smiles>

$40,52 \%$<smiles>CC(=O)N[C@@H](Cc1ccc(F)cc1F)OCc1ccccc1</smiles>

$4 p, 65 \%$<smiles>CC(=O)NC(Cc1cccc2ccccc12)C(=O)OCc1ccccc1</smiles>

$4 q, 52 \%$

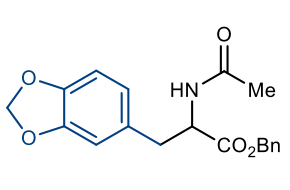

$4 r, 65 \%$<smiles>CC(=O)NC(Cc1ccccc1)Cc1ccccc1</smiles>

5d, $65 \%$<smiles>CC(=O)NC(Cc1ccccc1)C(=O)Nc1ccccc1</smiles>

5f, $71 \%$<smiles>CC(=O)NC(Cc1ccccc1)C(=O)NOc1ccccc1</smiles>

5g, $59 \%$

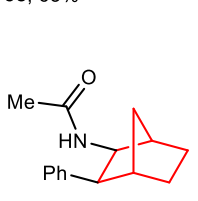

5i, $61 \%$

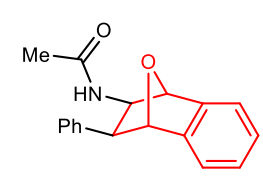

$\mathbf{5 j}, 89 \%$
$>20: 1 d r$<smiles>CC(=O)NC1C(c2ccccc2)C1(C)c1ccccc1</smiles><smiles>CC(=O)NC1C2c3ccccc3C(c3ccccc3)C2N1C(=O)OC(C)(C)C</smiles>

$\mathbf{5 k}, 55 \%$
$>20: 1 d r$

5I, $43 \%$ $12: 1 d r$

Dioxazolone scope<smiles>C=CCCCC(=O)NC(Cc1ccccc1)C(=O)OCc1ccccc1</smiles>

Reactions were conducted on a $0.1 \mathrm{mmol}$ scale using 1 (2.5 equiv), 2 (3.0 equiv), and $\mathbf{3}$ (1.0 equiv). Yields of isolated products after purification by chromatography are reported. Diastereoselectivity was determined by analysis of ${ }^{1} \mathrm{H}$ NMR of the unpurified reaction mixture. 
In addition, monitoring the reaction progress with ${ }^{1} \mathrm{H}$ NMR indicates that the reaction with dioxazolone that leads to product formation is faster than $\beta$-hydride elimination at the beginning of the reaction. The amount of $\beta$-hydride elimination product increases after consumption of limiting dioxazolone (3a) (see supporting information for the details).

In order to determine the turnover limiting step of the reaction, we investigated the alkene migratory insertion step with benzyl acrylate (2a) and deuterated benzyl acrylate (2a- $\left.\boldsymbol{d}_{\mathbf{2}}\right)$. The inverse secondary kinetic isotope effect (KIE) value of 0.72 measured from the intermolecular competition experiment (Scheme 3c) or 0.55 calculated by comparing the initial rate of two separate reactions, one with benzyl acrylate (2a) and one with deuterated benzyl acrylate (2a-d $\boldsymbol{d}_{2}$ (see supporting information for details) suggest $\mathrm{sp}^{2}$ to $\mathrm{sp}^{3}$ hybridization change occurs during the turnover limiting step of the reaction (Scheme 3c) ${ }^{33,34}$ In order to determine the relative stereochemistry of the product, we conducted the reaction with mono-deuterated benzyl acrylate (2a-d $\boldsymbol{d}_{1}, 85: 14$ E/Z) (Scheme 3d). Interestingly, the reaction gives the product $\left(\mathbf{4 a -}-\boldsymbol{d}_{1}\right)$ containing almost identical deuterium incorporation with starting alkene (2a-d $\left.\boldsymbol{d}_{\mathbf{1}}\right)$ which suggests the carboamination is highly diastereoselective. The product was cyclized by Pd-catalyzed intramolecular C-H amidation, ${ }^{35}$ which confirmed syn-carboamination of the alkene.

Based on these experiments, we propose the following mechanism for the reaction (Scheme 4). First, the active monomeric Rh complex I undergoes transmetalation with phenylboronic acid (1a) to give Rh-phenyl complex II. Next, turnover limiting migratory insertion into the Rh-aryl bond forms Rh(III) intermediate IV. Subsequently, dioxazolone (3a) coordinates to the electron-rich alkyl Rh(III) complex (IV) followed by $\mathrm{Rh}(\mathrm{V})$-nitrene formation with the exclusion of $\mathrm{CO}_{2}$. The desired syn-carboamination product (4a) is formed after reductive elimination and proto-demetallation with the regeneration of active $\mathrm{Rh}$ catalyst $\mathbf{I}$. 


\section{Scheme 3. Mechanistic investigation}

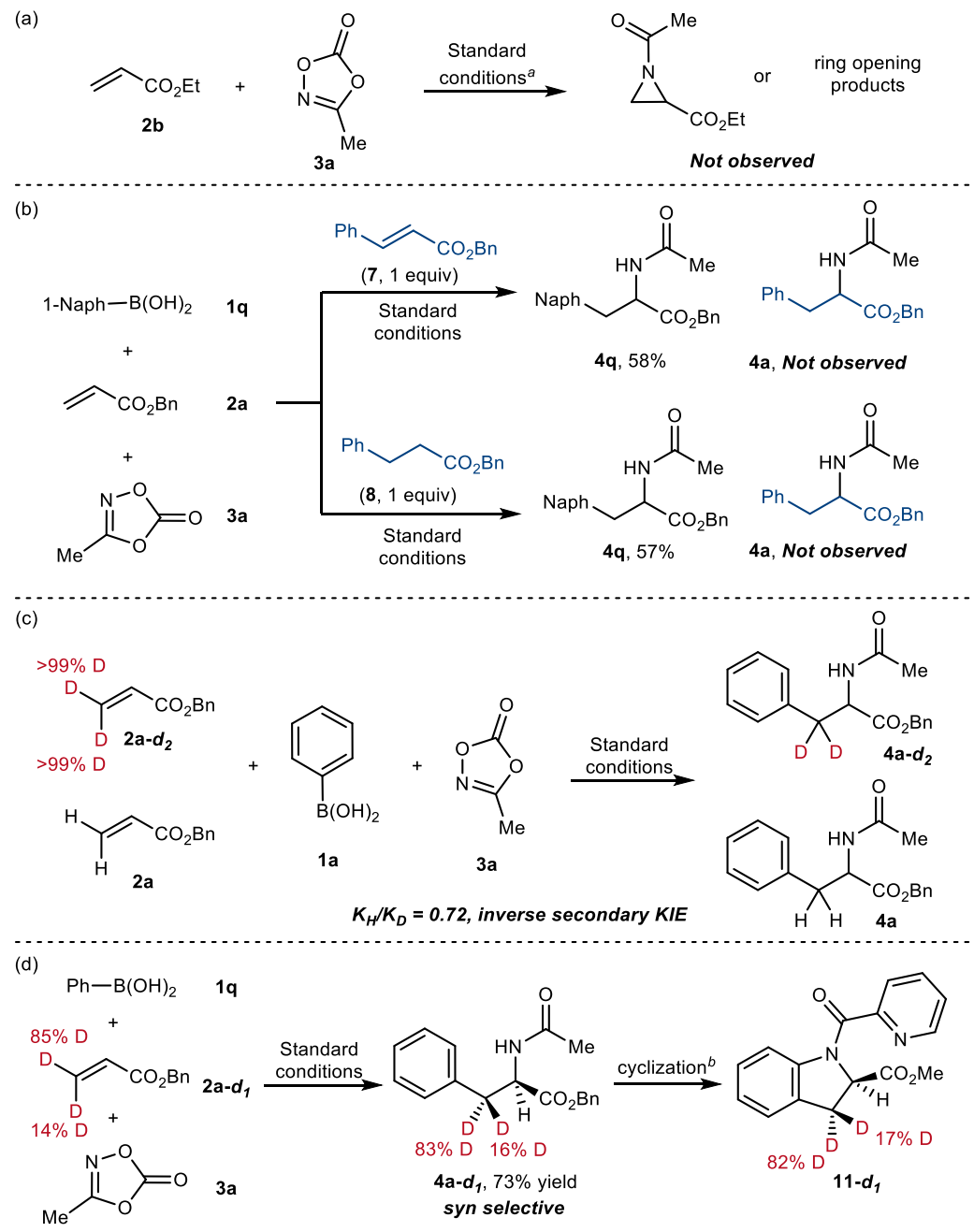

${ }^{a}$ Standard conditions: $\left[\mathrm{Cp}^{*} \mathrm{RhCl}_{2}\right]_{2}(5 \mathrm{~mol} \%), \mathrm{NaHCO}_{3}(30 \mathrm{~mol} \%), \mathrm{MeOH}(0.6 \mathrm{M})$, dioxazolone (1 equiv), arylboronic acid (2.5 equiv), alkene (3 equiv), $\left.25^{\circ} \mathrm{C} .{ }^{b} 1\right) 6 \mathrm{M} \mathrm{HCl}$; 2) $\mathrm{SOCl}_{2}$, $\mathrm{MeOH}$; 3 ) Picolinic acid, EDCI, HOBt, DCM; 4) $\mathrm{Pd}(\mathrm{OAc})_{2}, \mathrm{PhI}(\mathrm{OAc})_{2}, \mathrm{DCM}$. 


\section{Scheme 4. Proposed mechanism}

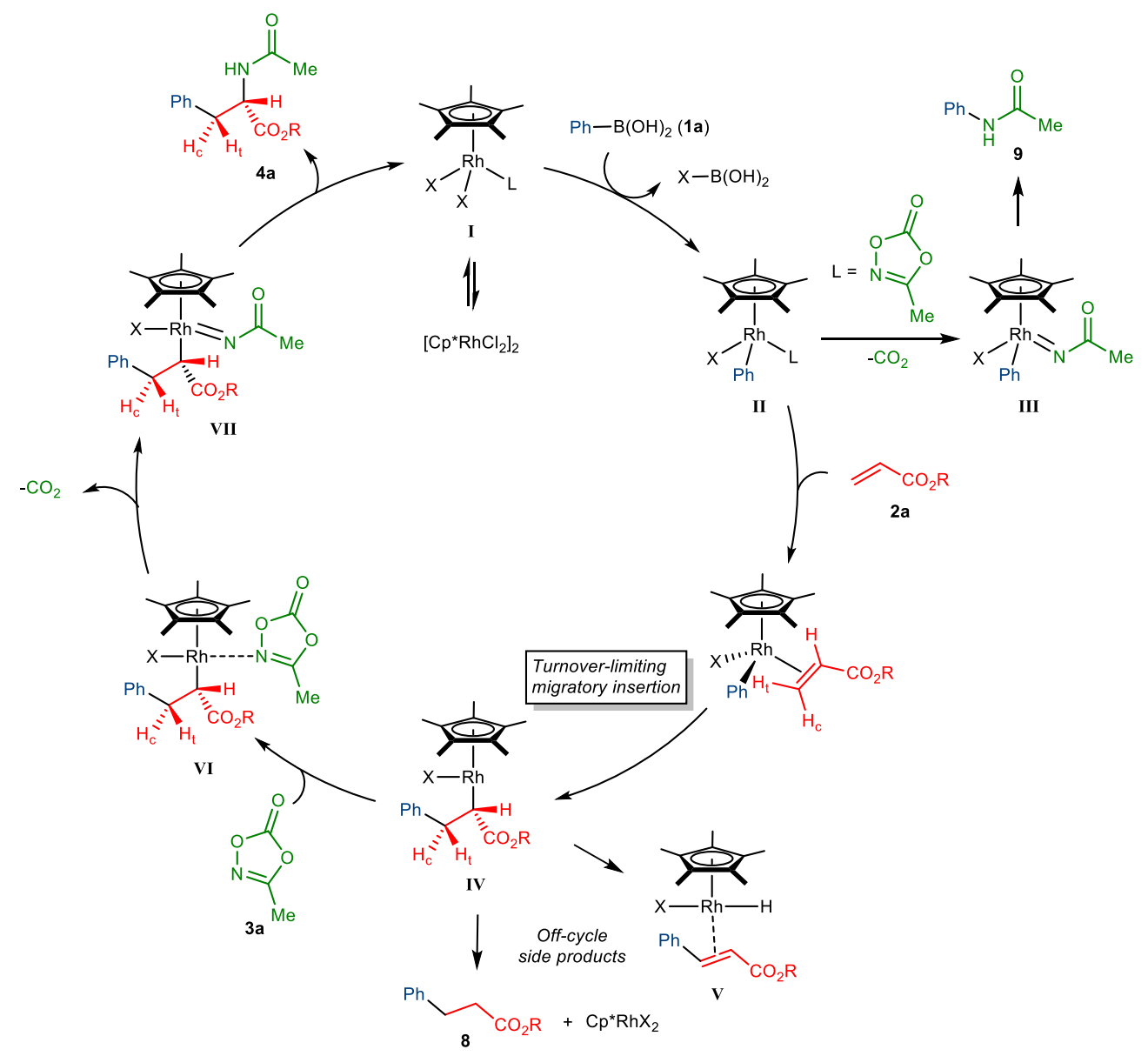

\section{CONCLUSIONS}

In summary, we have developed $\mathrm{Rh}(\mathrm{III})$-catalyzed 3-component regioselective syn-carboamination of alkenes. The reaction shows a broad scope with a variety of commercially available arylboronic acids and dioxazolones easily prepared from carboxylic acids. Mechanistic investigations suggest alkene migratory insertion to be the turnover limiting step supported by secondary KIE. In addition, deuterium labeling experiments provide experimental evidence for stereoselective syn-carboamination process. This method provides a rapid access to valuable amines including non-natural $\alpha$-amino acids from abundant alkenes with excellent regioselectivity and high diastereoselectivity with internal cyclic alkenes. 


\section{Methods}

\section{General procedure for the carboamination of alkenes}

$\left[\mathrm{Cp}^{*} \mathrm{RhCl}_{2}\right]_{2}(0.0050 \mathrm{mmol}, 5 \mathrm{~mol} \%)$, sodium bicarbonate $(0.03 \mathrm{mmol}, 0.3$ equiv), and boronic acid $(0.25 \mathrm{mmol}$, 2.5 equiv) were measured in a 0.5 -dram vial with a magnetic stir bar. In a separate vial, dioxazolone $(0.1 \mathrm{mmol}, 1$ equiv) and alkene ( $0.3 \mathrm{mmol}, 3$ equiv) were dissolved in $0.10 \mathrm{~mL}$ of $\mathrm{MeOH}$ and transferred to the first vial. It was rinsed with an additional $0.07 \mathrm{~mL}$ of $\mathrm{MeOH}$ and transferred again to the first vial. The reaction mixture was then stirred at $25{ }^{\circ} \mathrm{C}$ for 16 hours. After the completion of the reaction, the volatiles were removed by a rotary evaporator. Ethyl acetate was added to the residue and filtered through a short pad of celite and washed with ethyl acetate three

times. The filtrate was concentrated by rotary evaporator and a crude ${ }^{1} \mathrm{H}$ NMR spectrum was collected with $1,3,5-$ trimethoxybenzene as the internal standard. Purification was performed by flash chromatography or preparative TLC using ethyl acetate and hexane or DCM and $\mathrm{MeOH}$ as the eluent.

Acknowledgments: We gratefully acknowledge NIGMS (GM80442) for support.

Author contributions: S.L. and T.R. conceived the project. S.L. designed and performed all the experiments and data interpretation. T.R. supervised the project. S.L. and T.R. co-wrote the manuscript.

Competing Interests: The authors declare no competing interests. 
Author Information Reprints and permissions information is available at www.nature.com/reprints. The authors declare no competing financial interests. Correspondence and requests for materials should be addressed to T.R. (tr2504@,columbia.edu).

Abbreviations Cp*, pentamethylcyclopentadienyl.

\section{Data Availability}

The authors declare that the data supporting the findings of this study are available within the paper and its Supplementary Information or from the authors upon reasonable request.

\section{References:}

(1) Kolb, H. C., VanNieuwenhze, M. S. \& Sharpless, K. B. Catalytic Asymmetric Dihydroxylation. Chem. Rev. 94, 2483-2547 (1994).

(2) McDonald, R. I., Liu, G. \& Stahl, S. S. Palladium(II)-Catalyzed Alkene Functionalization via Nucleopalladation: Stereochemical Pathways and Enantioselective Catalytic Applications. Chem. Rev. 111, 2981-3019 (2011).

(3) Jensen, K. H. \& Sigman, M. S. Mechanistic Approaches to Palladium-Catalyzed Alkene Difunctionalization Reactions. Org. Biomol. Chem. 6, 4083-4088 (2008).

(4) Chemler, S. R. The Enantioselective Intramolecular Aminative Functionalization of Unactivated Alkenes, Dienes, Allenes and Alkynes for the Synthesis of Chiral Nitrogen Heterocycles. Org. Biomol. Chem. 7, 30093019 (2009). 
(5) Romero, R. M., Wöste, T. H. \& Muñiz, K. Vicinal Difunctionalization of Alkenes with Iodine(III) Reagents and Catalysts. Chem. - An Asian J. 9, 972-983 (2014).

(6) Cardona, F. \& Goti, A. Metal-Catalysed 1,2-Diamination Reactions. Nat. Chem. 1, 269-275 (2009).

(7) Vitaku, M. E., Smith, D. T. \& Njardarson, J. T. Analysis of the Structural Diversity, Substitution Patterns, and Frequency of Nitrogen Heterocycles among U.S. FDA Approved Pharmaceuticals. J. Med. Chem. 57, 1025710274 (2014).

(8) Jiang, H. \& Studer, A. Intermolecular Radical Carboamination of Alkenes. Chem. Soc. Rev. 49, 1790-1811 (2020).

(9) Coldham, I. \& Hufton, R. Intramolecular Dipolar Cycloaddition Reactions of Azomethine Ylides. Chem. Rev. 105, 2765-2810 (2005).

(10) Nakamura, I. \& Yamamoto, Y. Transition-Metal-Catalyzed Reactions in Heterocyclic Synthesis. Chem. Rev. 104, 2127-2198 (2004).

(11) Wolfe, J. P. Synthesis of saturated heterocycles via metal-catalyzed alkene carboamination or carboalkoxylation reactions. Top. Heterocycl. Chem. 32, 1-37 (2013).

(12) Mai, D. N. \& Wolfe, J. P. Asymmetric Palladium-Catalyzed Carboamination Reactions for the Synthesis of Enantiomerically Enriched 2-(Arylmethyl)- and 2-(Alkenylmethyl)Pyrrolidines. J. Am. Chem. Soc. 132, 1215712159 (2010).

(13) Zeng, W. \& Chemler, S. R. Copper(II)-Catalyzed Enantioselective Intramolecular Carboamination of Alkenes. J. Am. Chem. Soc. 129, 12948-12949 (2007).

(14) Piou, T. \& Rovis, T. Rhodium-Catalysed Syn-Carboamination of Alkenes via a Transient Directing Group. Nature 527, 86-90 (2015).

(15) Lerchen, A., Knecht, T., Daniliuc, C. G. \& Glorius, F. Unnatural Amino Acid Synthesis Enabled by the Regioselective Cobalt(III)-Catalyzed Intermolecular Carboamination of Alkenes. Angew. Chem. Int. Ed. 55, 15166-15170 (2016). 
(16) In the context of a mechanistic investigation, Glorius and coworkers observed carboamination product as a side product in low yield; see: Wang, X., Gensch, T., Lerchen, A., Daniliuc, C. G., \& Glorius, F. Cp*Rh(III)/Bicyclic Olefin Cocatalyzed C-H Bond Amidation by Intramolecular Amide Transfer. J. Am.

Chem. Soc. 139, 6506-6512 (2017).

(17) Ozols, K., Onodera, S., Woźniak, Ł. \& Cramer, N. Cobalt(III)-Catalyzed Enantioselective Intermolecular Carboamination by C-H Functionalization. Angew. Chem. Int. Ed. 60, 655-659 (2021).

(18) Maity, S., Potter, T.J. \& Ellman, J.A. $\alpha$-Branched amines by catalytic 1,1-addition of C-H bonds and aminating agents to terminal alkenes. Nat Catal 2, 756-762 (2019).

(19) Liu, Z. et al. Catalytic Intermolecular Carboamination of Unactivated Alkenes via Directed Aminopalladation. J. Am. Chem. Soc. 139, 11261-11270 (2017).

(20) van der Puyl, V. A., Derosa, J. \& Engle, K. M. Directed, Nickel-Catalyzed Umpolung 1,2-Carboamination of Alkenyl Carbonyl Compounds. ACS Catal. 9, 224-229 (2019).

(21) Wang, D. et al. Asymmetric Copper-Catalyzed Intermolecular Aminoarylation of Styrenes: Efficient Access to Optical 2,2-Diarylethylamines. J. Am. Chem. Soc. 139, 6811-6814 (2017).

(22) Gockel, S. N., Buchanan, T. L. \& Hull, K. L. Cu-Catalyzed Three-Component Carboamination of Alkenes. J. Am. Chem. Soc. 140, 58-61 (2018).

(23) Monos, T. M., McAtee, R. C. \& Stephenson, C. R. J. Arylsulfonylacetamides as Bifunctional Reagents for Alkene Aminoarylation. Science 361, 1369-1373 (2018).

(24) Park, Y., Park, K. T., Kim, J. G. \& Chang, S. Mechanistic Studies on the Rh(III)-Mediated Amido Transfer Process Leading to Robust C-H Amination with a New Type of Amidating Reagent. J. Am. Chem. Soc. 137, 4534-4542 (2015).

(25) Shin, K., Kim, H. \& Chang, S. Transition-Metal-Catalyzed C-N Bond Forming Reactions Using Organic Azides as the Nitrogen Source: A Journey for the Mild and Versatile C-H Amination. Acc. Chem. Res. 48, 10401052 (2015). 
(26) Park, Y., Kim, Y. \& Chang, S. Transition Metal-Catalyzed C-H Amination: Scope, Mechanism, and Applications. Chem. Rev. 117, 9247-9301 (2017).

(27) Lee, S., Lei, H. \& Rovis, T. A Rh(III)-Catalyzed Formal [4+1] Approach to Pyrrolidines from Unactivated Terminal Alkenes and Nitrene Sources. J. Am. Chem. Soc. 141, 12536-12540 (2019).

(28) Fagnou, K. \& Lautens. M. Rhodium-Catalyzed Carbon-Carbon Bond Forming Reactions of Organometallic Compounds. Chem. Rev. 103, 169-196 (2003).

(29) Hayashi, T. \& Yamasaki, K. Rhodium-Catalyzed Asymmetric 1,4-Addition and Its Related Asymmetric Reactions. Chem. Rev. 103, 2829-2844 (2003).

(30) Edwards, H. J., Hargrave, J. D., Penrosea, S. D. \& Frost, C. G. Synthetic applications of rhodium catalysed conjugate addition. Chem. Soc. Rev. 39, 2093-2105 (2010).

(31) Hong, S. Y. et al. Selective Formation of $\gamma$-Lactams via C-H Amidation Enabled by Tailored Iridium Catalysts. Science 359, 1016- 1021 (2018).

(32) Lee, S., Jang, Y.-J., Phipps, E. J. T., Lei, H. \& Rovis, T. Rhodium(III)-Catalyzed Three Component 1,2Diamination of Unactivated Terminal Alkenes. Synthesis 52, 1247-1252 (2020).

(33) Gómez-Gallego, M. \& Sierra, M. A. Kinetic Isotope Effects in the Study of Organometallic Reaction Mechanisms. Chem. Rev. 111, 4857-4963 (2011).

(34) Simmons, E. M. \& Hartwig, J. F. On the Interpretation of Deuterium Kinetic Isotope Effects in C-H Bond Functionalizations by Transition-Metal Complexes. Angew. Chem. Int. Ed. 51, 3066-3072 (2012).

(35) He, G., Lu, C., Zhao, Y., Nack, W. A. \& Chen, G. Improved Protocol for Indoline Synthesis via PalladiumCatalyzed Intramolecular C(Sp2)-H Amination. Org. Lett. 14, 2944-2947 (2012). 


\title{
Supporting Information
}

\section{Rh(III)-Catalyzed Three-Component syn-Carboamination of Alkenes Using Arylboronic Acids and Dioxazolones}

\author{
Sumin Lee and Tomislav Rovis* \\ Department of Chemistry, Columbia University, New York, New York 10027
}

\section{Contents}

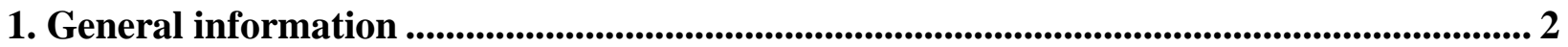

2. Preparation of Starting Materials …................................................................................................ 2

3. General procedure for the carboamination of alkenes............................................................... 5

4. Detailed optimization table .................................................................................................................... 6

5. Product characterization................................................................................................................... 11

6. Mechanistic studies .......................................................................................................................... 32

7. Limitation ........................................................................................................................................................ 45

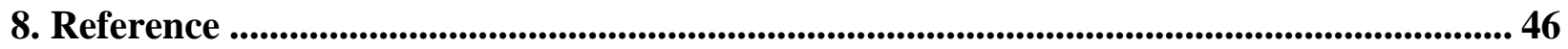

9. NMR Spectra .................................................................................................................................. 48 


\section{General information}

All reactions were carried out using oven-dried glassware with magnetic stirring unless otherwise noted. Anhydrous solvents and reagents were purchased from commercial sources and used without further purification. Flash chromatography was conducted either manually on SiliCycle ${ }^{\circledR}$ SilicaFlash $^{\circledR}$ P60 (230400 mesh) silica gel, SiliCycle ${ }^{\circledR}$ prep TLC (TLG-R10011B-341, thickness 1000um) or automatically via a Teledyne Isco Lumen CombiFlash with RediSep Rf Disposable Flash columns. Thin layer chromatography (TLC) was performed on Silicycle $250 \mu \mathrm{m}$ silica gel 60 Å plates. Visualization was accomplished with UV light (254 nm) and $\mathrm{KMnO}_{4} \cdot{ }^{1} \mathrm{H},{ }^{19} \mathrm{~F}$ NMR, and ${ }^{13} \mathrm{C}$ NMR spectra were collected at ambient temperature on Bruker $400 \mathrm{MHz}$ or Bruker Avance III $500 \mathrm{MHz}$ spectrometers unless otherwise noted. Chemical shifts $(\delta)$ are reported in parts per million (ppm), coupling constants $(J)$ are reported in $\mathrm{Hz}$, and multiplicity is described using the following abbreviations: singlet (s), broad (b), multiplet (m), doublet (d), triplet (t), quartet (q), or combinations thereof. ${ }^{1} \mathrm{H}$ NMR spectra were referenced to $7.26 \mathrm{ppm}\left(\mathrm{CHCl}_{3}\right)$ or $0.0 \mathrm{ppm}$ (TMS). ${ }^{13} \mathrm{C}$ NMR spectra were referenced to $77.16 \mathrm{ppm}\left(\mathrm{CDCl}_{3}\right)$ and all peaks given are singlet unless otherwise noted. Diastereomeric and regioisomeric ratios were measured by integration of ${ }^{1} \mathrm{H}$ NMR spectra of product mixtures prior to purification. High resolution mass spectra (HRMS) were obtained from Columbia University Mass Spectrometry Facility on a JOEL JMSHX110HF mass spectrometer using the ASAP ionization model. Infrared spectra were collected on a Perkin Elmer Spectrum Two FT-IR Spectrometer.

\section{Preparation of Starting Materials}

All boronic acids (1a-1r), alkenes $\mathbf{2 b}, \mathbf{2 c}, \mathbf{2 d}, \mathbf{2 e}$, and $\mathbf{2 i}$ were purchased from commercial sources and used without purification. Alkene $\mathbf{2 f},{ }^{1} \mathbf{2} \mathbf{j},{ }^{2} \mathbf{2} \mathbf{k},{ }^{3} \mathbf{2 l}^{4}, \mathbf{2} \mathbf{m}^{5}$ and dioxazolone $\mathbf{3 c},{ }^{6} \mathbf{3 d},{ }^{7} \mathbf{3 e},{ }^{8} \mathbf{3 f},{ }^{7} \mathbf{3 g},{ }^{6}$ and $\mathbf{3 h}{ }^{7}$ were synthesized following literatures. Alkenes $\mathbf{2 g}, \mathbf{2 h}$, and dioxazolone $\mathbf{3 b}$ were synthesized following the procedures below.

\section{General procedure A: the synthesis of acrylamides (2g-2h)}

To a solution of primary amine (or corresponding $\mathrm{HCl}$ salt, 1 equiv) and triethylamine (1.1 equiv) in dichloromethane $(0.5 \mathrm{M})$ was added acryloyl chloride $\left(1.1\right.$ equiv) in dichloromethane at $0{ }^{\circ} \mathrm{C}$. The mixture was stirred at $0{ }^{\circ} \mathrm{C}$ for 15 minutes and warmed to room temperature. The reaction was stirred at room temperature for 3 hours. The volatiles was removed by a rotary evaporator then ethyl acetate was added and filtered through celite. The filtrate was washed with $1 \mathrm{M} \mathrm{HCl}$, saturated $\mathrm{NaHCO}_{3}$ solution, and brine. 
The organic layer was dried over anhydrous $\mathrm{MgSO}_{4}$ and concentrated. The residue was purified by chromatography on silica gel (hexane/ethyl acetate) to afford the corresponding acrylamides.

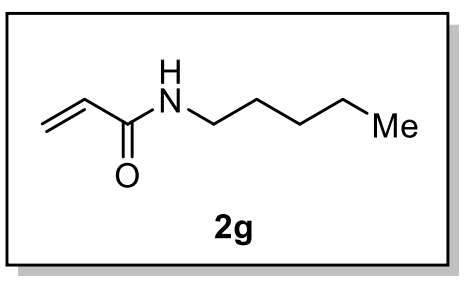

Yield: Prepared according to general procedure A from 1-aminopentane (10 mmol). 78\%, 1.11g, colorless oil.

$\mathbf{R}_{f}=0.39(\mathrm{EA} / \mathrm{Hex} 2: 3)$.

${ }^{1} \mathbf{H}$ NMR $\left(400 \mathrm{MHz}, \mathrm{CDCl}_{3}\right) \delta 6.23(\mathrm{~d}, J=17.1 \mathrm{~Hz}, 1 \mathrm{H}), 6.11(\mathrm{dd}, J=17.0,10.2 \mathrm{~Hz}, 1 \mathrm{H}), 6.05(\mathrm{~s}, 1 \mathrm{H})$, $5.58(\mathrm{dt}, J=10.0 \mathrm{~Hz}, 1 \mathrm{H}), 3.28(\mathrm{q}, J=7.3,6.7 \mathrm{~Hz}, 2 \mathrm{H}), 1.50(\mathrm{q}, J=7.2 \mathrm{~Hz}, 2 \mathrm{H}), 1.36-1.21(\mathrm{~m}, 4 \mathrm{H}), 0.87$ (t, $J=6.8,3 \mathrm{H})$.

${ }^{13}$ C NMR (101 MHz, $\left.\mathrm{CDCl}_{3}\right) \delta 165.75,131.22,125.99,39.70,29.33,29.18,22.43,14.04$.

IR $\left(\mathrm{CDCl}_{3}, \mathrm{~cm}^{-1}\right) v$ 3276, 3079, 2957, 2929, 2861, 1656, 1623, 1548, 1407, 1245, 985, 954, 727.

HRMS (ASAP) m/z $\left[\mathrm{C}_{8} \mathrm{H}_{16} \mathrm{NO}\right]^{+}\left([\mathrm{M}+\mathrm{H}]^{+}\right)$calculated 142.1232, found 142.1236.

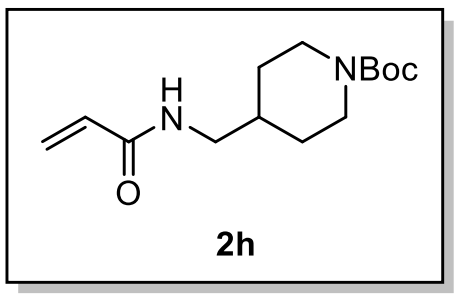

Yield: Prepared according to general procedure A from 1-Boc-4-(aminomethyl)piperidine (10 mmol). 65\%, $1.74 \mathrm{~g}$, colorless oil.

$\mathbf{R}_{f}=0.18(\mathrm{EA} / \operatorname{Hex} 2: 3)$.

${ }^{1}$ H NMR $\left(400 \mathrm{MHz}, \mathrm{CDCl}_{3}\right) \delta 6.26(\mathrm{dd}, J=17.0,1.6 \mathrm{~Hz}, 1 \mathrm{H}), 6.10(\mathrm{dd}, J=17.0,10.2 \mathrm{~Hz}, 1 \mathrm{H}), 6.06(\mathrm{~s}$, $1 \mathrm{H}), 5.61(\mathrm{dd}, J=10.2,1.6 \mathrm{~Hz}, 1 \mathrm{H}), 4.16-3.97(\mathrm{~m}, 2 \mathrm{H}), 3.30-3.12(\mathrm{~m}, 2 \mathrm{H}), 2.65(\mathrm{t}, J=12.7 \mathrm{~Hz}, 2 \mathrm{H})$, $1.73-1.61(\mathrm{~m}, 3 \mathrm{H}), 1.42(\mathrm{~s}, 9 \mathrm{H}), 1.19-1.04(\mathrm{~m}, 2 \mathrm{H})$.

${ }^{13}$ C NMR $\left(126 \mathrm{MHz}, \mathrm{CDCl}_{3}\right) \delta 165.82,154.95,130.86,126.74,79.57,45.10,43.76,36.57,29.92,28.59$. 
IR $\left(\mathrm{CDCl}_{3}, \mathrm{~cm}^{-1}\right) v 3302,3080,2976,2926,2854,2244,1658,1546,1424,1365,1226,1167,1143,906$, $727,647$.

HRMS (ESI) m/z $\left[\mathrm{C}_{14} \mathrm{H}_{24} \mathrm{~N}_{2} \mathrm{NaO}_{3}\right]^{+}\left([\mathrm{M}+\mathrm{Na}]^{+}\right)$calculated 291.1685, found 291.1699.

\section{Preparation of 3c}

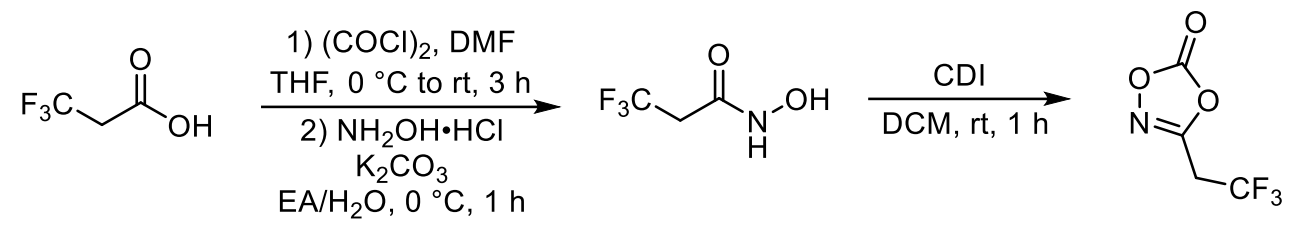

Oxalyl chloride (9.8 mmol, 0.98 equiv) and catalytic amount (5 - 10 drops) of DMF were added dropwise to a solution of 3,3,3-trifluoropropanoic acid (10 mmol, 1.0 equiv) in THF $(0.3 \mathrm{M})$ at $0{ }^{\circ} \mathrm{C}$. The reaction mixture was warmed to room temperature and stirred for 3 hours. After the full conversion of acid, the crude material was taken without concentration. In a separate flask, hydroxylamine hydrochloride (11 mmol, 1.1 equiv) was dissolved in $0.1 \mathrm{M}$ of ethyl acetate/ $\mathrm{H}_{2} \mathrm{O}$ 2:1 biphasic mixture. $\mathrm{K}_{2} \mathrm{CO}_{3}$ (20 mmol, 2 equiv) was added to the solution and cooled to $0{ }^{\circ} \mathrm{C}$. The crude THF solution of acid chloride was added dropwise to the mixture and stirred for 1 hour at $0{ }^{\circ} \mathrm{C}$. After completion of the reaction, the reaction mixture was extracted with ethyl acetate. The combined organic layers were washed with brine, dried over anhydrous $\mathrm{MgSO}_{4}$, filtered, and concentrated. The residue was used without further purification. To a solution of 3,3,3trifluoro- $N$-hydroxypropanamide (3.22 mmol, 1.0 equiv) in DCM (0.2 M), CDI (3.53 mmol, 1.2 equiv) was added as one portion. After stirring for 1 hour at room temperature, the reaction was quenched with $1 \mathrm{M}$ $\mathrm{HCl}$ and extracted with DCM three times. The combined organic layer was washed with brine and dried over sodium sulfate. The solvent was removed under reduced pressure to afford 3-(2,2,2-trifluoroethyl)1,4,2-dioxazol-5-one (3c). No further purification was required.

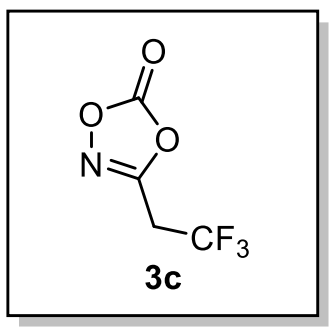

Yield: 44\% (over 2 steps), $2.05 \mathrm{mmol}, 346 \mathrm{mg}$, colorless oil. 
${ }^{1} \mathbf{H}$ NMR $\left(400 \mathrm{MHz}, \mathrm{CDCl}_{3}\right) \delta 3.56(\mathrm{q}, J=9.1 \mathrm{~Hz}, 2 \mathrm{H})$.

${ }^{13}$ C NMR $\left(101 \mathrm{MHz}, \mathrm{CDCl}_{3}\right) \delta 158.61(\mathrm{q}, J=3.9 \mathrm{~Hz}), 153.03,122.37(\mathrm{q}, J=278.2 \mathrm{~Hz}), 31.48(\mathrm{q}, J=34.9$ $\mathrm{Hz})$.

${ }^{19}$ F NMR $\left(376 \mathrm{MHz}, \mathrm{CDCl}_{3}\right) \delta-63.40(\mathrm{t}, J=9.1 \mathrm{~Hz})$.

\section{General procedure for the carboamination of alkenes}

$\left[\mathrm{Cp}^{*} \mathrm{RhCl}_{2}\right]_{2}(0.0050 \mathrm{mmol}, 5 \mathrm{~mol} \%)$, sodium bicarbonate $(0.03 \mathrm{mmol}, 0.3$ equiv), and boronic acid $(0.25$ mmol, 2.5 equiv) were measured in a 0.5 -dram vial with a magnetic stir bar. In a separate vial, dioxazolone ( $0.1 \mathrm{mmol}, 1$ equiv) and alkene $(0.3 \mathrm{mmol}, 3$ equiv) were dissolved in $0.10 \mathrm{~mL}$ of $\mathrm{MeOH}$ and transferred to the first vial. It was rinsed with an additional $0.07 \mathrm{~mL}$ of $\mathrm{MeOH}$ and transferred again to the first vial. The reaction mixture was then stirred at $25^{\circ} \mathrm{C}$ for 16 hours. After the completion of the reaction, the solvent was removed by a rotary evaporator. Ethyl acetate was added to the residue and filtered through a short pad of celite and washed with ethyl acetate three times. The filtrate was concentrated by rotary evaporator and a crude ${ }^{1} \mathrm{H}$ NMR spectrum was collected with 1,3,5-trimethoxybenzene as the internal standard. Purification was performed by flash chromatography or preparational TLC using ethyl acetate and hexane or DCM and $\mathrm{MeOH}$ as the eluent. 


\section{Detailed optimization table}

\section{Table S1. ${ }^{a}$}

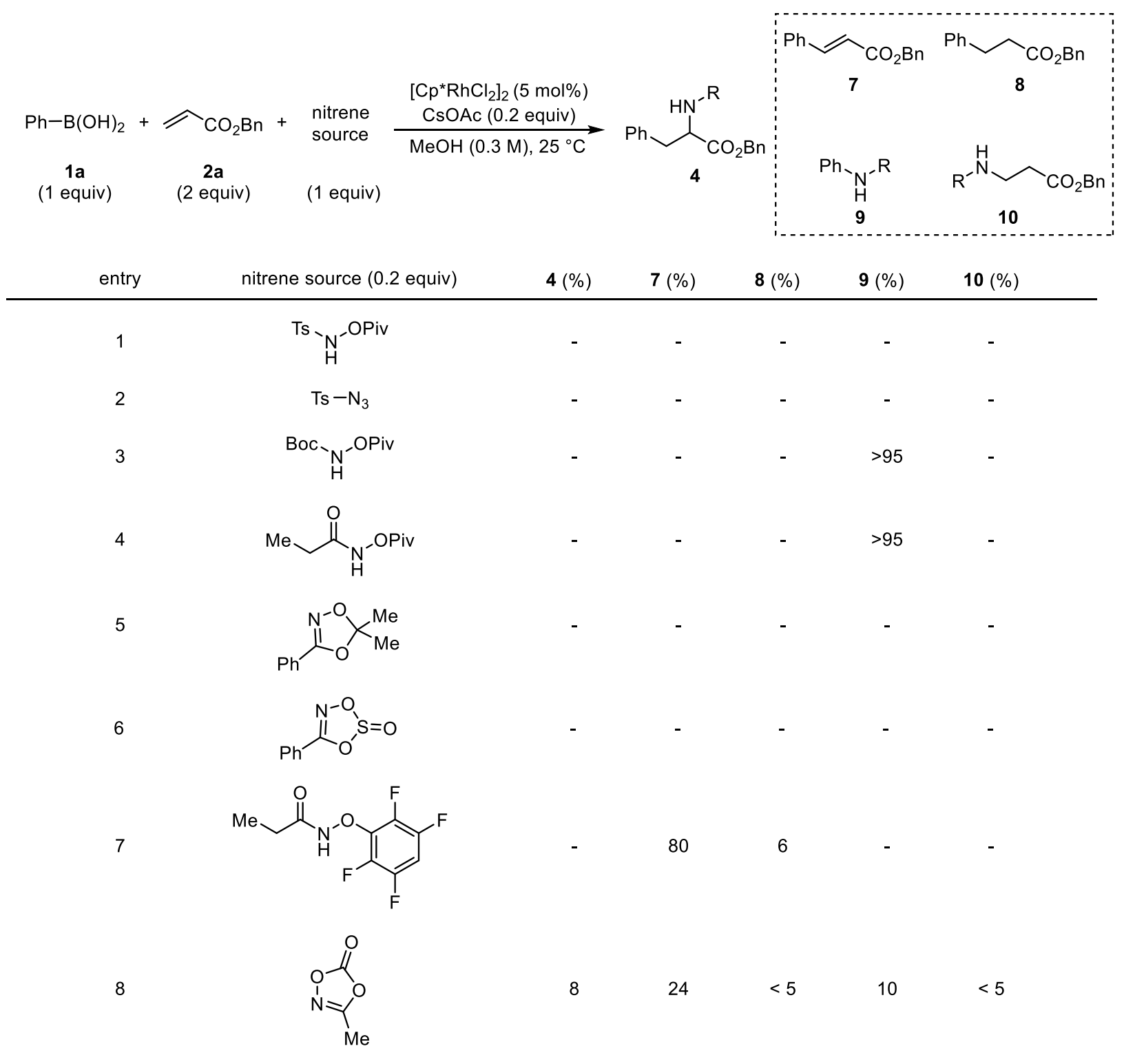

${ }^{a}$ Reaction were conducted on $0.1 \mathrm{mmol}$ scale according to the general procedure. Yield was determined by ${ }^{1} \mathrm{H} \mathrm{NMR}$ spectroscopy of the crude reaction mixture using 1,3,5-trimethoxybenzene as an internal standard. The yield of side products was calculated based on $0.1 \mathrm{mmol}$. 
Table S2. ${ }^{a}$

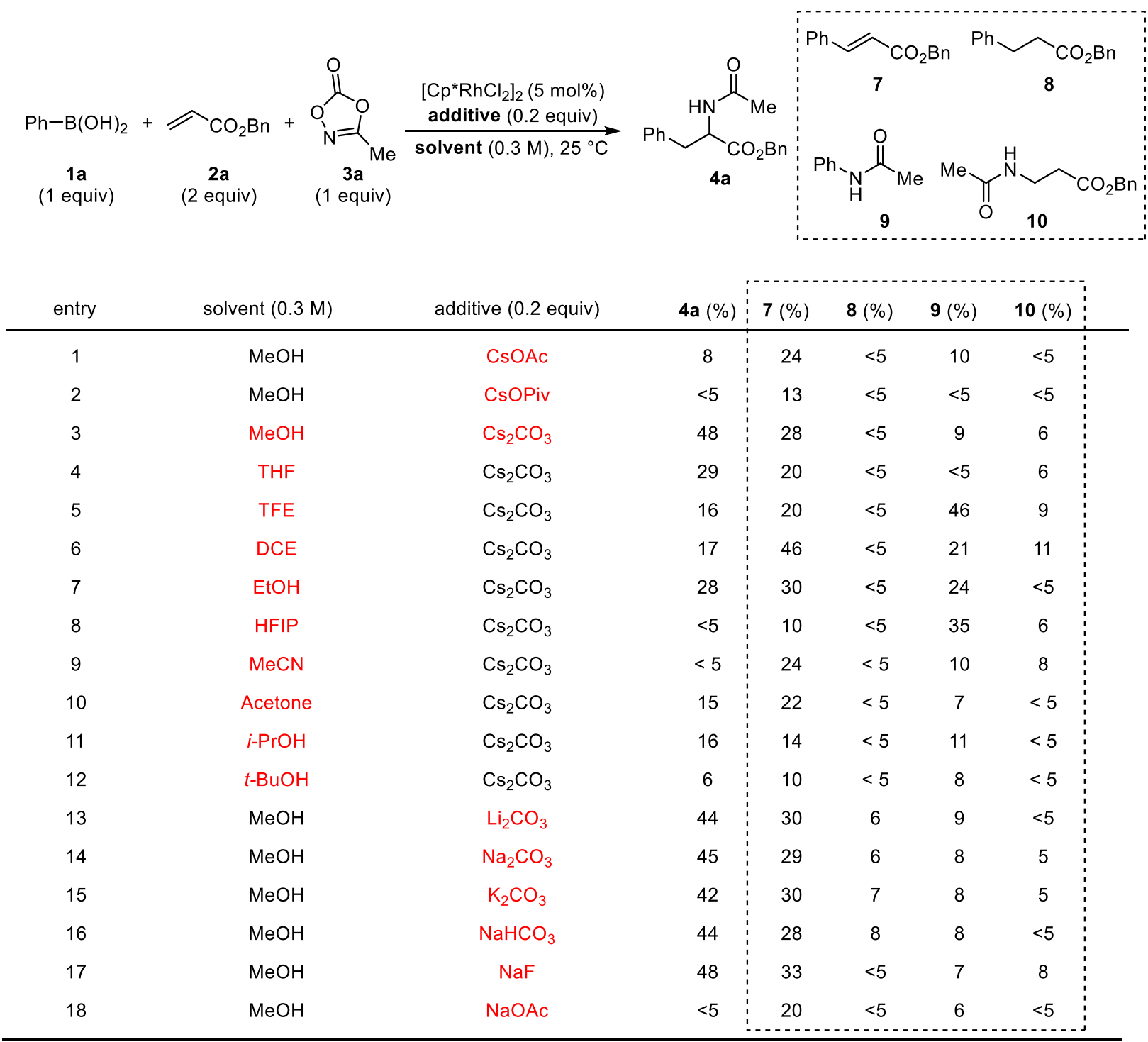

${ }^{a}$ Reaction were conducted on $0.1 \mathrm{mmol}$ scale according to the general procedure. Yield was determined by ${ }^{1} \mathrm{H}$ NMR spectroscopy of the crude reaction mixture using 1,3,5-trimethoxybenzene as an internal standard. The yield of side products was calculated based on $0.1 \mathrm{mmol}$. 


\section{Table S3. ${ }^{a}$}

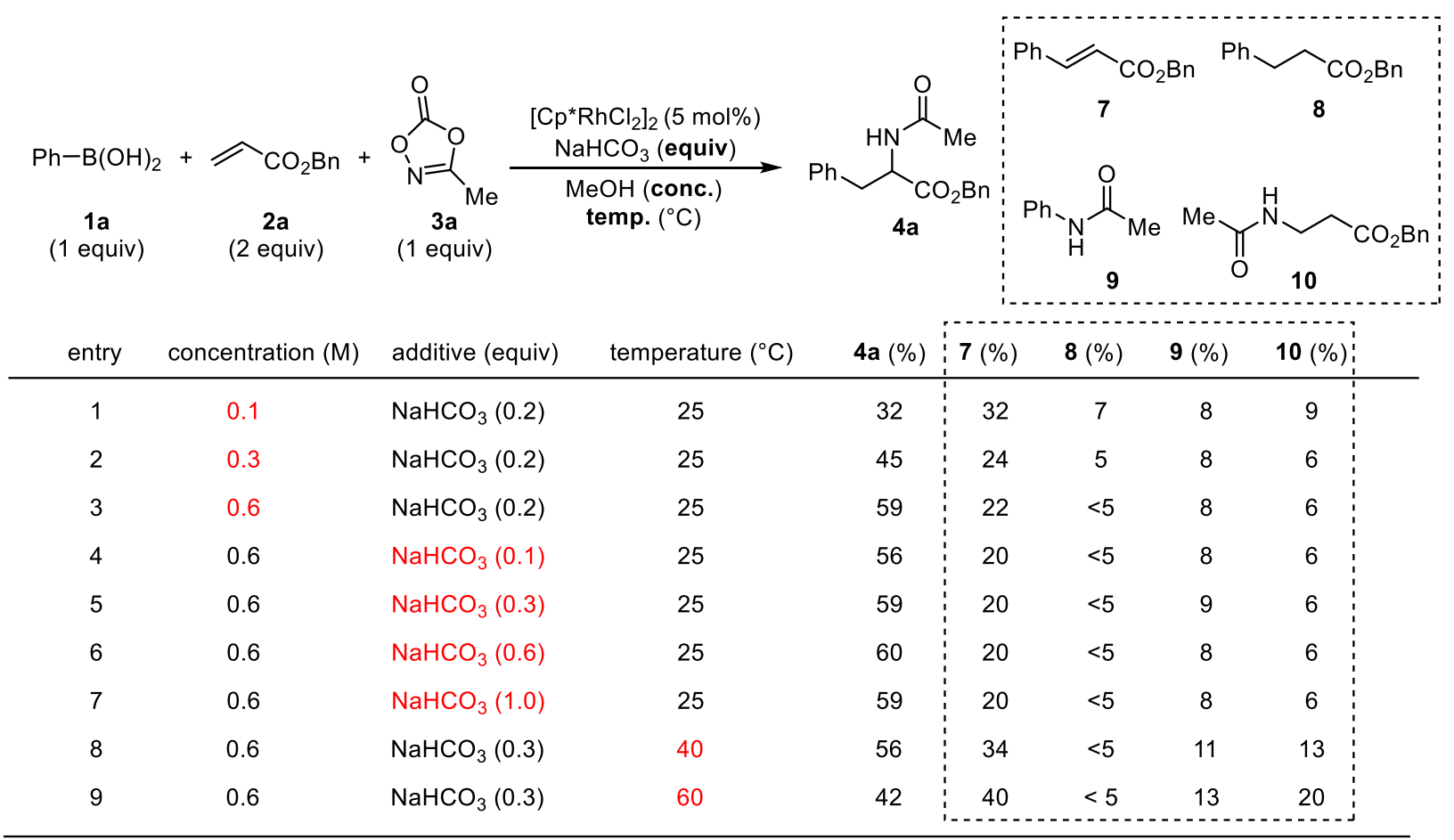

${ }^{a}$ Reaction were conducted on $0.1 \mathrm{mmol}$ scale according to the general procedure. Yield was determined by ${ }^{1} \mathrm{H}$ NMR spectroscopy of the crude reaction mixture using 1,3,5-trimethoxybenzene as an internal standard. The yield of side products was calculated based on $0.1 \mathrm{mmol}$. 


\section{Table S4. ${ }^{a}$}

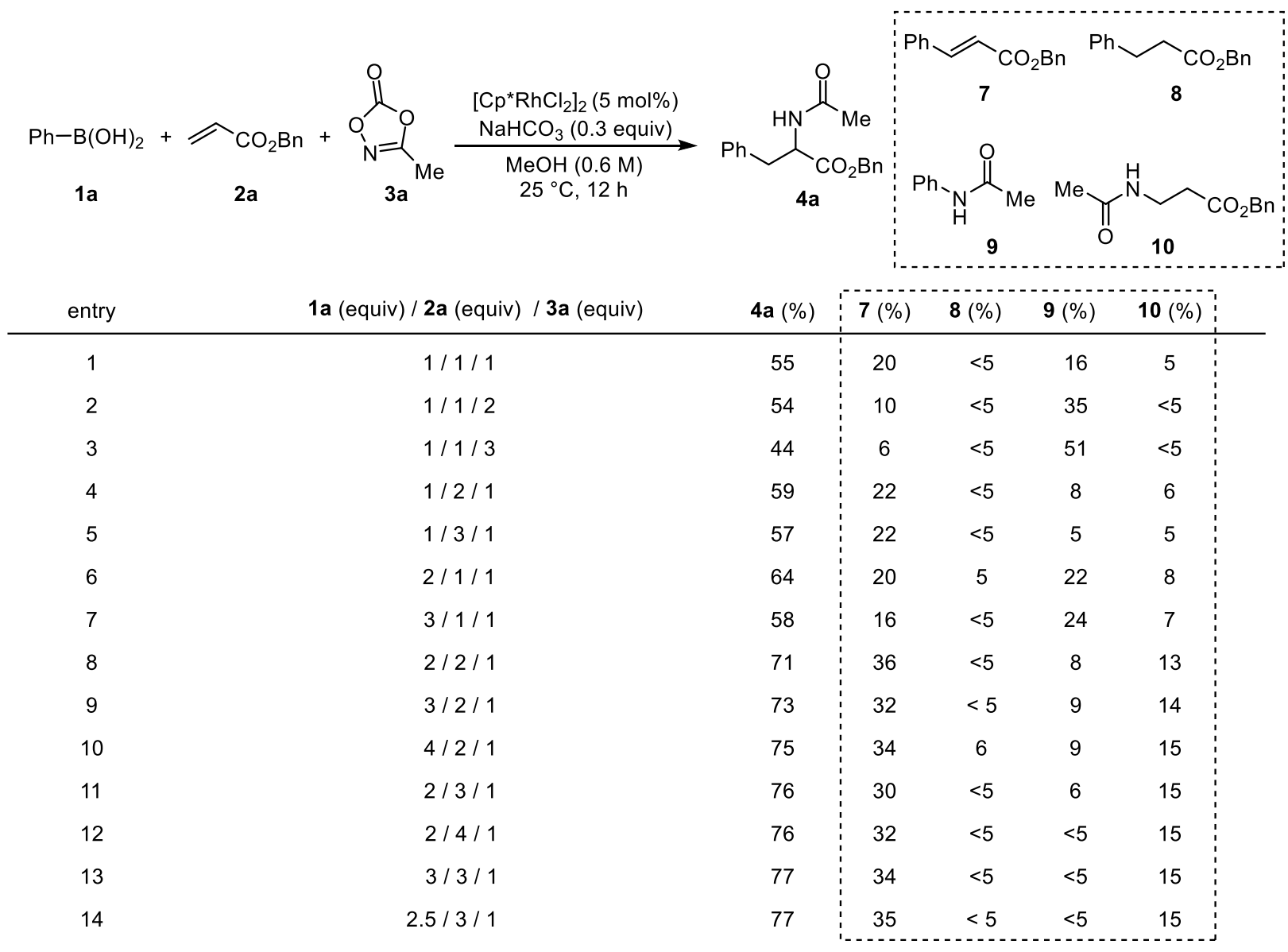

${ }^{a}$ Reaction were conducted on $0.1 \mathrm{mmol}$ scale according to the general procedure. Yield was determined by ${ }^{1} \mathrm{H}$ NMR spectroscopy of the crude reaction mixture using 1,3,5-trimethoxybenzene as an internal standard. The yield of side products was calculated based on $0.1 \mathrm{mmol}$.

\section{Table S5. ${ }^{a}$}

\begin{tabular}{|c|c|c|c|c|}
\hline \multirow[b]{3}{*}{ entry } & \multirow{3}{*}{$\begin{array}{c}+\mathrm{CO}_{2} \mathrm{Bn} \\
2 \mathrm{a} \\
(3.0 \text { equiv })\end{array}$} & \multirow{2}{*}{$+{ }_{\text {3a }}^{+}$Me equiv $)$} & \multirow{2}{*}{$\begin{array}{c}{\left[\mathrm{Cp}^{*} \mathrm{RhCl}_{2}\right]_{2}(5 \mathrm{~mol} \%)} \\
\mathrm{NaHCO}_{3}(0.3 \text { equiv }) \\
\mathrm{MeOH}(0.6 \mathrm{M}), 25^{\circ} \mathrm{C}\end{array}$} & $-\mathrm{HN}^{\prime}$ \\
\hline & & & & $4 a$ \\
\hline & & carbon source & (2.5 equiv) & yield (\%) \\
\hline 1 & & $\mathrm{Ph}-\mathrm{B}(\mathrm{C}$ & & 77 \\
\hline 2 & & $\mathrm{Ph}-\mathrm{B}^{\prime}$ & & 39 \\
\hline 3 & & $\mathrm{Ph}-\mathrm{BF}$ & & trace \\
\hline
\end{tabular}

${ }^{a}$ Reaction were conducted on $0.1 \mathrm{mmol}$ scale according to the general procedure. Yield was determined by ${ }^{1} \mathrm{H}$ NMR spectroscopy of the crude reaction mixture using $1,3,5-$ trimethoxybenzene as an internal standard. 
Table S6. Summary of the detailed optimization table.

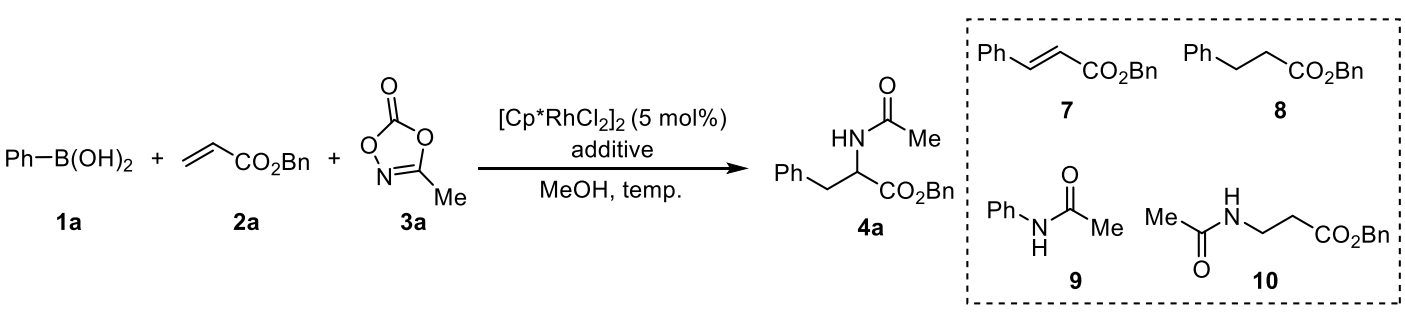

- Different nitrene sources give different side products.

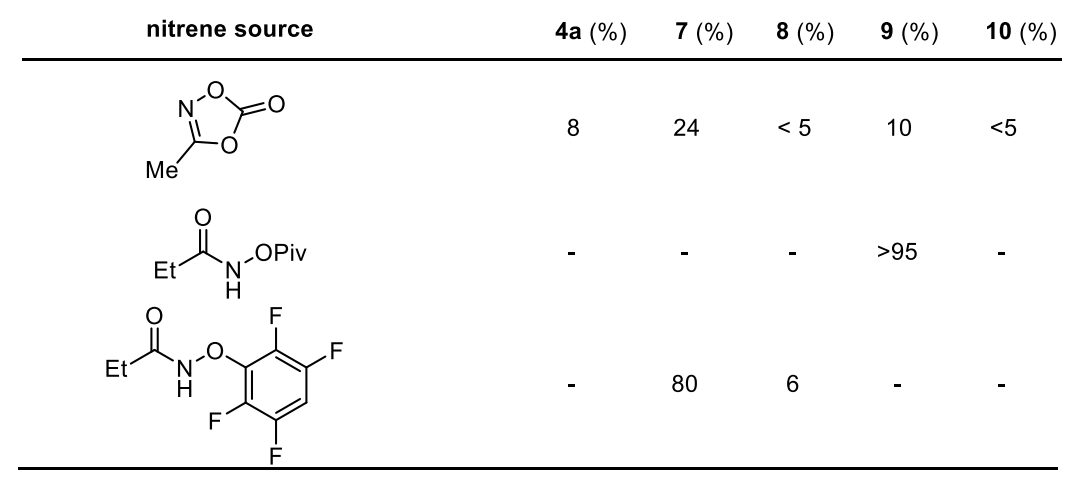

1a (1 equiv), 2a (2 equiv), 3a (1 equiv), $\mathrm{CsOAc}(0.2$ equiv), $\mathrm{MeOH}(0.3 \mathrm{M})$

- Acetates inhibit product formation, 7 becomes major product.

\begin{tabular}{llllll} 
additive & $\mathbf{4 a}(\%)$ & $\mathbf{7}(\%)$ & $\mathbf{8}(\%)$ & $\mathbf{9 ( \% )}$ & $\mathbf{1 0}(\%)$ \\
\hline $\mathrm{NaHCO}_{3}$ & 44 & 28 & 8 & 8 & 5 \\
$\mathrm{NaOAc}$ & $<5$ & 20 & $<5$ & 6 & $<5$ \\
\hline
\end{tabular}

1a (1 equiv), 2a (2 equiv), 3a (1 equiv), additive ( 0.2 equiv), $\mathrm{MeOH}(0.3 \mathrm{M})$

- Acidic solvent leads to more direct C-N coupling product (9).

\begin{tabular}{llllll} 
solvent & $\mathbf{4 a}(\%)$ & $\mathbf{7}(\%)$ & $\mathbf{8}(\%)$ & $\mathbf{9}(\%)$ & $\mathbf{1 0}(\%)$ \\
\hline $\mathrm{MeOH}$ & 48 & 28 & $<5$ & 9 & 6 \\
$\mathrm{TFE}$ & 16 & 20 & $<5$ & 46 & 9 \\
\hline
\end{tabular}

1a (1 equiv), 2 a (2 equiv), $3 \mathbf{a}$ (1 equiv), $\mathrm{Cs}_{2} \mathrm{CO}_{3}$ (0.3 equiv), $\mathrm{MeOH}(0.3 \mathrm{M})$

- Higher temperature give low chemoselectivity

\begin{tabular}{cccccc} 
temperature & $\mathbf{4 a}(\%)$ & $\mathbf{7}(\%)$ & $\mathbf{8}(\%)$ & $\mathbf{9 ( \% )}$ & $\mathbf{1 0}(\%)$ \\
\hline $25^{\circ} \mathrm{C}$ & 59 & 20 & $<5$ & 9 & 6 \\
$60{ }^{\circ} \mathrm{C}$ & 42 & 40 & $<5$ & 13 & 20 \\
\hline
\end{tabular}

1a (1 equiv), 2a (2 equiv), 3a (1 equiv), $\mathrm{NaHCO}_{3}(0.3$ equiv), $\mathrm{MeOH}(0.6 \mathrm{M})$ 


\section{Product characterization}

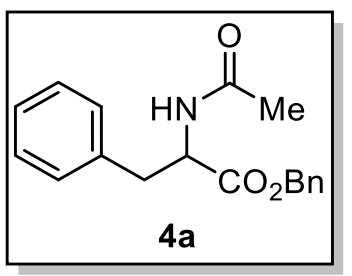

Yield: 68\%, $20 \mathrm{mg}$, white solid.

$\mathbf{R}_{f}=0.26(\mathrm{EA} / \operatorname{Hex} 2: 3)$.

${ }^{1}$ H NMR (400 MHz, $\left.\mathrm{CDCl}_{3}\right) \delta 7.41$ - $7.29(\mathrm{~m}, 3 \mathrm{H}), 7.31$ - $7.26(\mathrm{~m}, 2 \mathrm{H}), 7.26$ - 7.17 (m, 3H), 7.04 - 6.95 $(\mathrm{m}, 2 \mathrm{H}), 5.93(\mathrm{~d}, J=7.9 \mathrm{~Hz}, 1 \mathrm{H}), 5.17(\mathrm{~d}, J=12.1 \mathrm{~Hz}, 1 \mathrm{H}), 5.12(\mathrm{~d}, J=12.1 \mathrm{~Hz}, 1 \mathrm{H}), 4.92(\mathrm{dt}, J=7.9,5.7$ $\mathrm{Hz}, 1 \mathrm{H}), 3.18-3.03(\mathrm{~m}, 2 \mathrm{H}), 1.98$ (s, 3H).

${ }^{13}$ C NMR (101 MHz, $\left.\mathrm{CDCl}_{3}\right) \delta 171.62,169.68,135.82,135.17,129.43,128.76,128.72,128.69,128.67$, 127.20, 67.40, 53.26, 37.94, 23.27.

IR $\left(\mathrm{CDCl}_{3}, \mathrm{~cm}^{-1}\right) v$ 3277, 3063, 3031, 2932, 1738, 1652, 1540, 1372, 1209, 1173, 734, 696.

HRMS (ASAP) m/z $\left[\mathrm{C}_{18} \mathrm{H}_{20} \mathrm{NO}_{3}\right]^{+}\left([\mathrm{M}+\mathrm{H}]^{+}\right)$calculated 298.1443, found 298.1437.

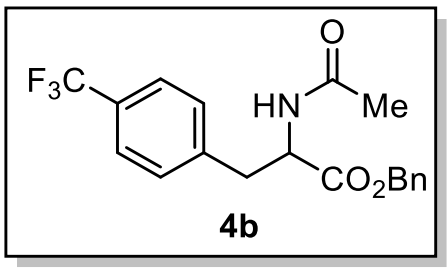

Yield: $62 \%, 23 \mathrm{mg}$, white solid.

$\mathbf{R}_{f}=0.38(\mathrm{EA} / \mathrm{Hex} 2: 3)$.

${ }^{1} \mathbf{H}$ NMR $\left(400 \mathrm{MHz}, \mathrm{CDCl}_{3}\right) \delta 7.44(\mathrm{~d}, J=8.0 \mathrm{~Hz}, 2 \mathrm{H}), 7.40-7.34(\mathrm{~m}, 3 \mathrm{H}), 7.31-7.24(\mathrm{~m}, 2 \mathrm{H}), 7.07(\mathrm{~d}$, $J=7.9 \mathrm{~Hz}, 2 \mathrm{H}), 5.98(\mathrm{~d}, J=7.3 \mathrm{~Hz}, 1 \mathrm{H}), 5.19(\mathrm{~d}, J=12.0 \mathrm{~Hz}, 1 \mathrm{H}), 5.09(\mathrm{~d}, J=12.0 \mathrm{~Hz}, 1 \mathrm{H}), 4.97-4.90$ (m, 1H), $3.23-3.11(\mathrm{~m}, 2 \mathrm{H}), 1.99(\mathrm{~s}, 3 \mathrm{H})$.

${ }^{13}$ C NMR $\left(101 \mathrm{MHz}, \mathrm{CDCl}_{3}\right) \delta 171.28,169.74,140.03(\mathrm{~d}, J=1.6 \mathrm{~Hz}), 134.89,129.80,129.45(\mathrm{q}, J=32.7$ $\mathrm{Hz}), 128.92,128.84,125.51(\mathrm{q}, J=3.7 \mathrm{~Hz}), 124.17(\mathrm{q}, J=270.9 \mathrm{~Hz}), 67.66,53.10,37.80,23.28$.

${ }^{19} \mathbf{F}$ NMR $\left(376 \mathrm{MHz}, \mathrm{CDCl}_{3}\right) \delta-62.51$. 
IR $\left(\mathrm{CDCl}_{3}, \mathrm{~cm}^{-1}\right) v 3276,3066,3036,2939,1740,1654,1541,1324,1164,1122,1067,904,729,699$.

HRMS (ASAP) m/z $\left[\mathrm{C}_{19} \mathrm{H}_{19} \mathrm{~F}_{3} \mathrm{NO}_{3}\right]^{+}\left([\mathrm{M}+\mathrm{H}]^{+}\right)$calculated 366.1317, found 366.1310.

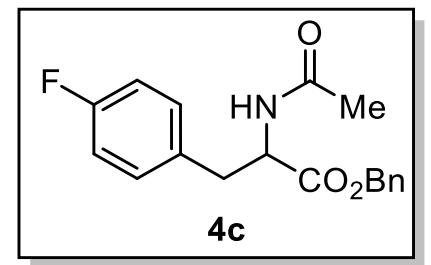

Yield: 60\%, $20 \mathrm{mg}$, white solid

$\mathbf{R}_{f}=0.25(\mathrm{EA} / \mathrm{Hex} 2: 3)$.

${ }^{1} \mathbf{H}$ NMR $\left(400 \mathrm{MHz}, \mathrm{CDCl}_{3}\right) \delta 7.40-7.34(\mathrm{~m}, 3 \mathrm{H}), 7.33-7.19(\mathrm{~m}, 2 \mathrm{H}), 6.96-6.84(\mathrm{~m}, 4 \mathrm{H}), 5.97(\mathrm{~d}, J=$ $7.7 \mathrm{~Hz}, 1 \mathrm{H}), 5.19(\mathrm{~d}, J=12.0 \mathrm{~Hz}, 1 \mathrm{H}), 5.10(\mathrm{~d}, J=12.1 \mathrm{~Hz}, 1 \mathrm{H}), 4.89(\mathrm{dt}, J=7.8,5.7 \mathrm{~Hz}, 1 \mathrm{H}), 3.15-3.00$ $(\mathrm{m}, 2 \mathrm{H}), 1.98(\mathrm{~s}, 3 \mathrm{H})$.

${ }^{13} \mathrm{C}$ NMR $\left(101 \mathrm{MHz}, \mathrm{CDCl}_{3}\right) \delta 171.49,169.69,162.08(\mathrm{~d}, J=245.4 \mathrm{~Hz}), 135.05,131.51(\mathrm{~d}, J=3.3 \mathrm{~Hz})$, $130.91(\mathrm{~d}, J=8.0 \mathrm{~Hz}), 128.85,128.81,115.48$ (d, $J=21.3 \mathrm{~Hz}), 67.50,53.30,37.17,23.28$. (Two aromatic peaks overlapped).

${ }^{19}$ F NMR $\left(376 \mathrm{MHz}, \mathrm{CDCl}_{3}\right) \delta-115.54--115.76(\mathrm{~m})$.

IR $\left(\mathrm{CDCl}_{3}, \mathrm{~cm}^{-1}\right) v 3278,3066,2935,1738,1653,1541,1508,1373,1220,1176,905,828,729,698$.

HRMS (ASAP) m/z $\left[\mathrm{C}_{18} \mathrm{H}_{19} \mathrm{FNO}_{3}\right]^{+}\left([\mathrm{M}+\mathrm{H}]^{+}\right)$calculated 316.1349, found 316.1348.

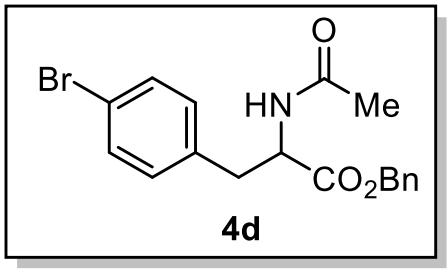

Yield: $65 \%, 25 \mathrm{mg}$, white solid.

$\mathbf{R}_{f}=0.31(\mathrm{EA} / \mathrm{Hex} 2: 3)$.

${ }^{1}$ H NMR $\left(400 \mathrm{MHz}, \mathrm{CDCl}_{3}\right) \delta 7.42-7.34(\mathrm{~m}, 3 \mathrm{H}), 7.33-7.25(\mathrm{~m}, 4 \mathrm{H}), 6.87-6.79(\mathrm{~m}, 2 \mathrm{H}), 5.96(\mathrm{~d}, J=$ $7.7 \mathrm{~Hz}, 1 \mathrm{H}), 5.19(\mathrm{~d}, J=12.0 \mathrm{~Hz}, 1 \mathrm{H}), 5.09(\mathrm{~d}, J=12.1 \mathrm{~Hz}, 1 \mathrm{H}), 4.89(\mathrm{dt}, J=7.8,5.7 \mathrm{~Hz}, 1 \mathrm{H}), 3.13-2.99$ $(\mathrm{m}, 2 \mathrm{H}), 1.98(\mathrm{~s}, 3 \mathrm{H})$. 
${ }^{13}$ C NMR $\left(101 \mathrm{MHz}, \mathrm{CDCl}_{3}\right) \delta 171.38,169.70,134.99,134.84,131.73,131.14,128.87,128.85,128.83$, $121.21,67.54,53.11,37.40,23.26$.

IR $\left(\mathrm{CDCl}_{3}, \mathrm{~cm}^{-1}\right) v 3277,3063,3034,2930,1739,1652,1539,1488,1209,1128,1071,813,749$.

HRMS (ASAP) m/z [ $\left.\mathrm{C}_{18} \mathrm{H}_{19} \mathrm{BrNO}_{3}\right]^{+}\left([\mathrm{M}+\mathrm{H}]^{+}\right)$calculated 376.0548, found 376.0555.

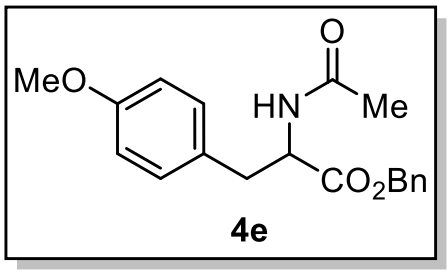

Yield: 60\%, $20 \mathrm{mg}$, white solid.

$\mathbf{R}_{f}=0.33(\mathrm{EA} / \mathrm{Hex} 2: 3)$.

${ }^{1} \mathbf{H}$ NMR $\left(400 \mathrm{MHz}, \mathrm{CDCl}_{3}\right) \delta 7.41-7.33(\mathrm{~m}, 3 \mathrm{H}), 7.33-7.27(\mathrm{~m}, 2 \mathrm{H}), 6.94-6.86(\mathrm{~m}, 2 \mathrm{H}), 6.78-6.70$ $(\mathrm{m}, 2 \mathrm{H}), 5.91(\mathrm{~d}, J=7.9 \mathrm{~Hz}, 1 \mathrm{H}), 5.17(\mathrm{~d}, J=12.1 \mathrm{~Hz}, 1 \mathrm{H}), 5.11(\mathrm{~d}, J=12.1 \mathrm{~Hz}, 1 \mathrm{H}), 4.88(\mathrm{dt}, J=7.9,5.7$ $\mathrm{Hz}, 1 \mathrm{H}), 3.76$ (s, 3H), $3.14-3.00(\mathrm{~m}, 2 \mathrm{H}), 1.97$ (s, 3H).

${ }^{13}$ C NMR (101 MHz, $\left.\mathrm{CDCl}_{3}\right) \delta 171.70,169.69,158.76,135.17,130.40,128.72,128.66,127.68,114.05$, $67.33,55.29,53.38,37.05,23.26$. (Two aromatic peaks overlapped)

IR $\left(\mathrm{CDCl}_{3}, \mathrm{~cm}^{-1}\right) v$ 3278, 3063, 3034, 2953, 2934, 2836, 1739, 1653, 1511, 1373, 1246, 1177, 1033, 698.

HRMS (ASAP) m/z [ $\left.\mathrm{C}_{19} \mathrm{H}_{22} \mathrm{NO}_{4}\right]^{+}\left([\mathrm{M}+\mathrm{H}]^{+}\right)$calculated 328.1549, found 328.1549.

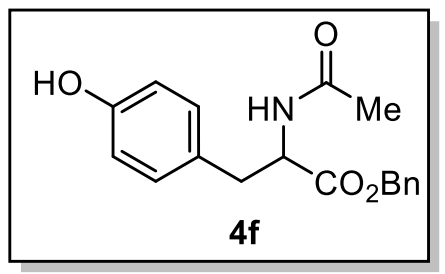

Yield: 70\%, $22 \mathrm{mg}$, white solid.

$\mathbf{R}_{f}=0.17(\mathrm{EA} / \mathrm{Hex} 2: 3)$.

${ }^{1}$ H NMR $\left(400 \mathrm{MHz}, \mathrm{CDCl}_{3}\right) \delta 7.41-7.23(\mathrm{~m}, 5 \mathrm{H}), 7.02(\mathrm{~s}, 1 \mathrm{H}), 6.87-6.79(\mathrm{~m}, 2 \mathrm{H}), 6.77-6.62(\mathrm{~m}, 2 \mathrm{H})$, $6.10(\mathrm{~d}, J=8.1 \mathrm{~Hz}, 1 \mathrm{H}), 5.18(\mathrm{~d}, J=12.1 \mathrm{~Hz}, 1 \mathrm{H}), 5.12(\mathrm{~d}, J=12.1 \mathrm{~Hz}, 1 \mathrm{H}), 4.90(\mathrm{dt}, J=8.0,5.9 \mathrm{~Hz}, 1 \mathrm{H})$, $3.06(\mathrm{dd}, J=14.1,5.8 \mathrm{~Hz}, 1 \mathrm{H}), 2.97$ (dd, $J=14.1,6.0 \mathrm{~Hz}, 1 \mathrm{H}), 1.97$ (s, 3H). 
${ }^{13} \mathrm{C}$ NMR $\left(101 \mathrm{MHz}, \mathrm{CDCl}_{3}\right) \delta 171.84,170.51,155.65,135.10,130.43,128.78,128.73,128.71,126.90$, $115.70,67.52,53.54,37.22,23.16$.

IR $\left(\mathrm{CDCl}_{3}, \mathrm{~cm}^{-1}\right) v$ 3298, 3062, 3033, 2930, 1737, 1652, 1514, 1451, 1374, 1213, 905, 730, 699.

HRMS (ASAP) m/z [ $\left.\mathrm{C}_{18} \mathrm{H}_{20} \mathrm{NO}_{4}\right]^{+}\left([\mathrm{M}+\mathrm{H}]^{+}\right)$calculated 314.1392, found 314.1390.

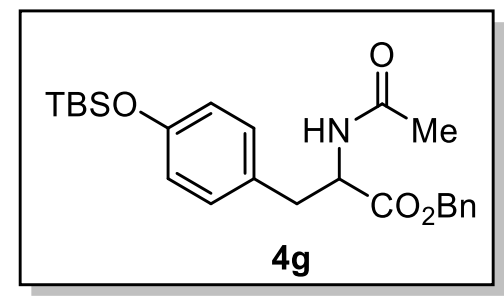

Yield: 66\%, $28 \mathrm{mg}$, colorless oil.

$\mathbf{R}_{f}=0.38(\mathrm{EA} / \operatorname{Hex} 2: 3)$.

${ }^{1}$ H NMR (400 MHz, $\left.\mathrm{CDCl}_{3}\right) \delta 7.41-7.29(\mathrm{~m}, 5 \mathrm{H}), 6.89-6.79(\mathrm{~m}, 2 \mathrm{H}), 6.73-6.63(\mathrm{~m}, 2 \mathrm{H}), 5.89(\mathrm{~d}, J=$ $7.9 \mathrm{~Hz}, 1 \mathrm{H}), 5.16(\mathrm{~d}, J=12.1 \mathrm{~Hz}, 1 \mathrm{H}), 5.11(\mathrm{~d}, J=12.2 \mathrm{~Hz}, 1 \mathrm{H}), 4.88(\mathrm{dt}, J=7.9,5.7 \mathrm{~Hz}, 1 \mathrm{H}), 3.11-2.96$ (m, 2H), 1.97 (s, 3H), 0.97 (s, 9H), 0.18 (s, 6H).

${ }^{13}$ C NMR $\left(101 \mathrm{MHz}, \mathrm{CDCl}_{3}\right) \delta 171.71,169.65,154.88,135.24,130.41,128.77,128.72,128.67,128.36$, $120.21,67.36,53.35,37.16,25.79,23.28,18.30,-4.29$.

IR $\left(\mathrm{CDCl}_{3}, \mathrm{~cm}^{-1}\right)$ v 3282, 3063, 3033, 2954, 2930, 2888, 2857, 1740, 1655, 1508, 1254, 906, 838, 728, 697. HRMS (ASAP) m/z [C $\left.{ }_{24} \mathrm{H}_{34} \mathrm{NO}_{4} \mathrm{Si}\right]^{+}\left([\mathrm{M}+\mathrm{H}]^{+}\right)$calculated 428.2257, found 428.2254.

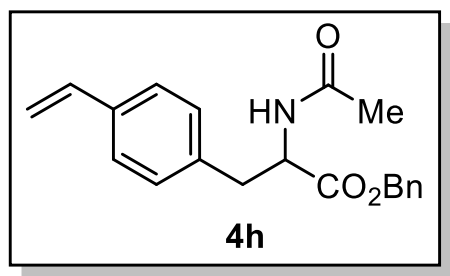

Yield: 55\%, $18 \mathrm{mg}$, white solid.

$\mathbf{R}_{f}=0.31(\mathrm{EA} / \operatorname{Hex} 2: 3)$.

${ }^{1}$ H NMR $\left(400 \mathrm{MHz}, \mathrm{CDCl}_{3}\right) \delta 7.39-7.33(\mathrm{~m}, 3 \mathrm{H}), 7.34-7.22(\mathrm{~m}, 4 \mathrm{H}), 6.99-6.91(\mathrm{~m}, 2 \mathrm{H}), 6.67(\mathrm{dd}, J$ $=17.6,10.9 \mathrm{~Hz}, 1 \mathrm{H}), 5.94(\mathrm{~d}, J=7.9 \mathrm{~Hz}, 1 \mathrm{H}), 5.70(\mathrm{dd}, J=17.6,1.0 \mathrm{~Hz}, 1 \mathrm{H}), 5.23(\mathrm{dd}, J=10.9,0.9 \mathrm{~Hz}$, 
$1 \mathrm{H}), 5.17(\mathrm{~d}, J=12.1 \mathrm{~Hz}, 1 \mathrm{H}), 5.11(\mathrm{~d}, J=12.1 \mathrm{~Hz}, 1 \mathrm{H}), 4.92(\mathrm{dt}, J=7.8,5.8 \mathrm{~Hz}, 1 \mathrm{H}), 3.17-3.03(\mathrm{~m}$, 2H), 1.98 (s, 3H).

${ }^{13}$ C NMR (101 MHz, $\left.\mathrm{CDCl}_{3}\right) \delta 171.59,169.72,136.58,136.51,135.40,135.12,129.61,128.77,128.74$, $128.70,126.50,113.90,67.43,53.25,37.69,23.27$.

IR $\left(\mathrm{CDCl}_{3}, \mathrm{~cm}^{-1}\right)$ v 3278, 3063, 2930, 2252, 1739, 1654, 1539, 1511, 1373, 1209, 1178, 904, 727, 699, 649. HRMS (ASAP) m/z $\left[\mathrm{C}_{20} \mathrm{H}_{22} \mathrm{NO}_{3}\right]^{+}\left([\mathrm{M}+\mathrm{H}]^{+}\right)$calculated 324.1600, found 324.1605.

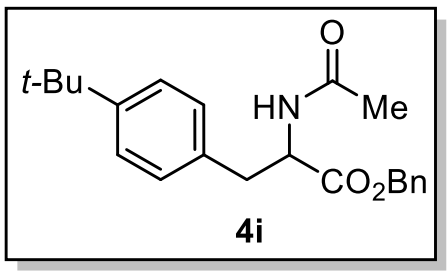

Yield: 55\%, $19 \mathrm{mg}$, white solid.

$\mathbf{R}_{f}=0.37(\mathrm{EA} / \mathrm{Hex} 2: 3)$.

${ }^{1} \mathbf{H}$ NMR $\left(400 \mathrm{MHz}, \mathrm{CDCl}_{3}\right) \delta 7.38$ - $7.33(\mathrm{~m}, 3 \mathrm{H}), 7.32$ - 7.27 (m, 2H), 7.26 - 7.22 (m, 2H), 6.97 - 6.91 $(\mathrm{m}, 2 \mathrm{H}), 5.93(\mathrm{~d}, J=7.7 \mathrm{~Hz}, 1 \mathrm{H}), 5.18-5.09(\mathrm{~m}, 2 \mathrm{H}), 4.92(\mathrm{dt}, J=7.9,5.7 \mathrm{~Hz}, 1 \mathrm{H}), 3.08(\mathrm{~d}, J=5.7 \mathrm{~Hz}$, 2H), 1.98 (s, 3H), 1.29 (s, 9H).

${ }^{13}$ C NMR $\left(101 \mathrm{MHz}, \mathrm{CDCl}_{3}\right) \delta 171.76,169.74,150.05,135.21,132.63,129.10,128.74,128.70,128.66$, $125.60,67.39,53.26,37.39,34.55,31.45,23.29$.

IR $\left(\mathrm{CDCl}_{3}, \mathrm{~cm}^{-1}\right) v 3280,3062,3033,2960,2868,1740,1654,1537,1515,1368,1190,906,730,697$. HRMS (ASAP) m/z [ $\left[\mathrm{C}_{22} \mathrm{H}_{28} \mathrm{NO}_{3}\right]^{+}\left([\mathrm{M}+\mathrm{H}]^{+}\right)$calculated 354.2069, found 354.2072.

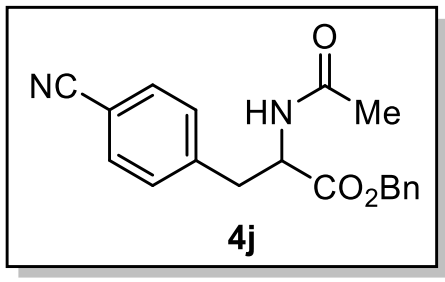

Yield: $51 \%, 16 \mathrm{mg}$, yellow oil. $\mathbf{R}_{f}=0.23(\mathrm{EA} / \mathrm{Hex} 1: 1)$. 
${ }^{1} \mathbf{H}$ NMR $\left(400 \mathrm{MHz}, \mathrm{CDCl}_{3}\right) \delta 7.48$ - $7.38(\mathrm{~m}, 2 \mathrm{H}), 7.42$ - 7.31 (m, 3H), 7.31 - $7.28(\mathrm{~m}, 2 \mathrm{H}), 7.08$ - 7.02 $(\mathrm{m}, 2 \mathrm{H}), 6.03(\mathrm{~d}, J=7.5 \mathrm{~Hz}, 1 \mathrm{H}), 5.22(\mathrm{~d}, J=12.0 \mathrm{~Hz}, 1 \mathrm{H}), 5.08(\mathrm{~d}, J=11.9 \mathrm{~Hz}, 1 \mathrm{H}), 4.92(\mathrm{dt}, J=7.5,5.8$ $\mathrm{Hz}, 1 \mathrm{H}), 3.16(\mathrm{qd}, J=13.8,5.9 \mathrm{~Hz}, 2 \mathrm{H}), 1.99(\mathrm{~s}, 3 \mathrm{H})$.

${ }^{13}$ C NMR (101 MHz, $\left.\mathrm{CDCl}_{3}\right) \delta 171.07,169.75,141.57,134.82,132.27,130.22,129.01,128.99,128.86$, $118.76,111.11,67.70,53.00,38.13,23.24$.

IR $\left(\mathrm{CDCl}_{3}, \mathrm{~cm}^{-1}\right) v 3283,3064,3035,2936,2228,1736,1656,1534,1505,1374,1268,1211,1175,905$, $731,698$.

HRMS (ASAP) m/z $\left[\mathrm{C}_{19} \mathrm{H}_{19} \mathrm{~N}_{2} \mathrm{O}_{3}\right]^{+}\left([\mathrm{M}+\mathrm{H}]^{+}\right)$calculated 323.1396, found 323.1390.

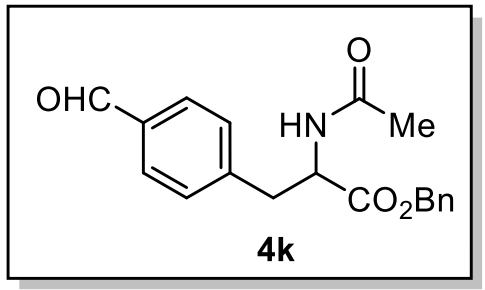

Yield: 64\%, $21 \mathrm{mg}$, white solid.

$\mathbf{R}_{f}=0.20(\mathrm{EA} / \mathrm{Hex} 2: 3)$.

${ }^{1} \mathbf{H}$ NMR $\left(400 \mathrm{MHz}, \mathrm{CDCl}_{3}\right) \delta 9.94(\mathrm{~s}, 1 \mathrm{H}), 7.73-7.66(\mathrm{~m}, 2 \mathrm{H}), 7.41-7.31(\mathrm{~m}, 3 \mathrm{H}), 7.32-7.27(\mathrm{~m}, 2 \mathrm{H})$, $7.17-7.10(\mathrm{~m}, 2 \mathrm{H}), 6.05(\mathrm{~d}, J=7.7 \mathrm{~Hz}, 1 \mathrm{H}), 5.20(\mathrm{~d}, J=12.1 \mathrm{~Hz}, 1 \mathrm{H}), 5.10(\mathrm{~d}, J=12.0 \mathrm{~Hz}, 1 \mathrm{H}), 4.96(\mathrm{dt}$, $J=7.7,5.9 \mathrm{~Hz}, 1 \mathrm{H}), 3.19(\mathrm{qd}, J=13.8,5.9 \mathrm{~Hz}, 2 \mathrm{H}), 1.99(\mathrm{~s}, 3 \mathrm{H})$.

${ }^{13}$ C NMR $\left(101 \mathrm{MHz}, \mathrm{CDCl}_{3}\right) \delta 191.90,171.23,169.76,143.15,135.40,134.90,130.15,129.97,128.90$, $128.89,128.83,67.63,53.06,38.20,23.24$.

IR $\left(\mathrm{CDCl}_{3}, \mathrm{~cm}^{-1}\right) v 3284,3062,3034,2932,2831,2738,2251,1738,1695,1656,1606,1534,1374,1306$, 1211, 1170, 905, 730, 698.

HRMS (ASAP) m/z $\left[\mathrm{C}_{19} \mathrm{H}_{20} \mathrm{NO}_{4}\right]^{+}\left([\mathrm{M}+\mathrm{H}]^{+}\right)$calculated 326.1392, found 326.1387.

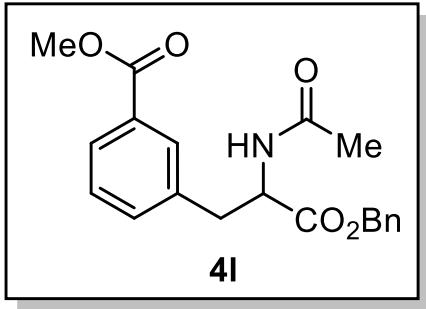


Yield: $65 \%, 23 \mathrm{mg}$, yellow oil.

$\mathbf{R}_{f}=0.19(\mathrm{EA} / \mathrm{Hex} 2: 3)$.

${ }^{1} \mathbf{H}$ NMR $\left(400 \mathrm{MHz}, \mathrm{CDCl}_{3}\right) \delta 7.90(\mathrm{dt}, J=7.7,1.5 \mathrm{~Hz}, 1 \mathrm{H}), 7.79(\mathrm{t}, J=1.8 \mathrm{~Hz}, 1 \mathrm{H}), 7.41-7.32(\mathrm{~m}, 3 \mathrm{H})$, $7.36-7.24(\mathrm{~m}, 3 \mathrm{H}), 7.19$ (dt, $J=7.7,1.5 \mathrm{~Hz}, 1 \mathrm{H}), 6.04(\mathrm{~d}, J=7.8 \mathrm{~Hz}, 1 \mathrm{H}), 5.28-5.09$ (m, 2H), 4.94 (dt, $J=7.7,5.9 \mathrm{~Hz}, 1 \mathrm{H}), 3.89$ (s, 3H), $3.20(\mathrm{dd}, J=13.8,6.0 \mathrm{~Hz}, 1 \mathrm{H}), 3.13(\mathrm{dd}, J=13.9,5.7 \mathrm{~Hz}, 1 \mathrm{H}), 1.99$ (s, $3 \mathrm{H})$.

${ }^{13}$ C NMR $\left(101 \mathrm{MHz}, \mathrm{CDCl}_{3}\right) \delta 171.42,169.79,166.94,136.35,134.99,133.92,130.58,130.49,128.77$, $128.73,128.68,128.46,67.57,53.28,52.25,37.79,23.22$. (Two aromatic peaks overlapped)

IR $\left(\mathrm{CDCl}_{3}, \mathrm{~cm}^{-1}\right)$ v 3282, 3064, 3034, 2951, 1719, 1655, 1537, 1436, 1283, 1202, 1109, 908, 747, 733, 697. HRMS (ASAP) m/z $\left[\mathrm{C}_{20} \mathrm{H}_{22} \mathrm{NO}_{5}\right]^{+}\left([\mathrm{M}+\mathrm{H}]^{+}\right)$calculated 356.1498, found 356.1502.

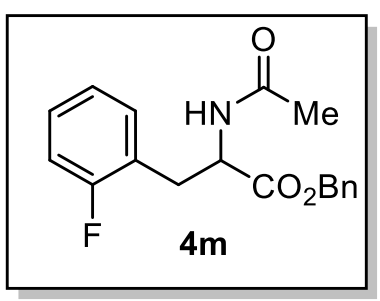

Yield: 74\%, $23 \mathrm{mg}$, yellow oil.

$\mathbf{R}_{f}=0.45(\mathrm{EA} / \mathrm{Hex} 2: 3)$.

${ }^{1} \mathbf{H}$ NMR $\left(400 \mathrm{MHz}, \mathrm{CDCl}_{3}\right) \delta 7.01-7.33(\mathrm{~m}, 3 \mathrm{H}), 7.33-7.28(\mathrm{~m}, 2 \mathrm{H}), 7.24-7.17(\mathrm{~m}, 1 \mathrm{H}), 7.08-6.95$ $(\mathrm{m}, 3 \mathrm{H}), 6.04(\mathrm{~d}, J=8.0 \mathrm{~Hz}, 1 \mathrm{H}), 5.17(\mathrm{~d}, J=12.2,1 \mathrm{H}), 5.12(\mathrm{~d}, J=12,2 \mathrm{~Hz}, 1 \mathrm{H}), 4.91(\mathrm{dt}, J=8.0,6.0$ $\mathrm{Hz}, 1 \mathrm{H}), 3.22(\mathrm{ddd}, J=13.9,6.0,1.2 \mathrm{~Hz}, 1 \mathrm{H}), 3.12(\mathrm{ddd}, J=13.9,6.1,1.1 \mathrm{~Hz}, 1 \mathrm{H}), 1.96(\mathrm{~s}, 3 \mathrm{H})$.

${ }^{13}$ C NMR $\left(101 \mathrm{MHz}, \mathrm{CDCl}_{3}\right) \delta 171.52,169.79,161.48(\mathrm{~d}, J=245.2 \mathrm{~Hz}), 135.17,131.80(\mathrm{~d}, J=5.1 \mathrm{~Hz})$, $129.12(\mathrm{~d}, J=8.3 \mathrm{~Hz}), 128.73,128.64,128.60,124.32(\mathrm{~d}, J=3.9 \mathrm{~Hz}), 123.02$ (d, $J=16.0 \mathrm{~Hz}), 115.49$ (d, $J=22.5 \mathrm{~Hz}), 67.56,52.67,31.52(\mathrm{~d}, J=1.8 \mathrm{~Hz}), 23.17$.

${ }^{19}$ F NMR $\left(376 \mathrm{MHz}, \mathrm{CDCl}_{3}\right) \delta-117.50(\mathrm{~m})$.

IR $\left(\mathrm{CDCl}_{3}, \mathrm{~cm}^{-1}\right) v 3277,3064,3036,2936,1739,1654,1539,1493,1454,1373,1271,1209,1230,1176$ $1132,755,697$.

HRMS (ASAP) m/z [ $\left.\mathrm{C}_{18} \mathrm{H}_{19} \mathrm{FNO}_{3}\right]^{+}\left([\mathrm{M}+\mathrm{H}]^{+}\right)$calculated 316.1349 , found 316.1350. 


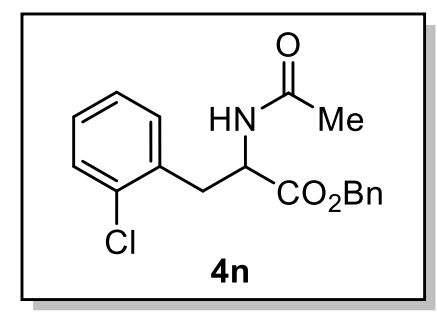

Yield: 37\%, $12 \mathrm{mg}$, colorless liquid.

$\mathbf{R}_{f}=0.26(\mathrm{EA} / \mathrm{Hex} 2: 3)$.

${ }^{1}$ H NMR (400 MHz, $\left.\mathrm{CDCl}_{3}\right) \delta 7.40-7.24(\mathrm{~m}, 6 \mathrm{H}), 7.21-7.05(\mathrm{~m}, 3 \mathrm{H}), 6.01(\mathrm{~d}, J=8.2 \mathrm{~Hz}, 1 \mathrm{H}), 5.17(\mathrm{~d}$, $J=12.2 \mathrm{~Hz}, 1 \mathrm{H}), 5.12(\mathrm{~d}, J=12.2 \mathrm{~Hz}, 1 \mathrm{H}), 4.96(\mathrm{ddd}, J=8.2,7.1,6.3 \mathrm{~Hz}, 1 \mathrm{H}), 3.32(\mathrm{dd}, J=13.9,6.3 \mathrm{~Hz}$, $1 \mathrm{H}), 3.18(\mathrm{dd}, J=13.9,7.1 \mathrm{~Hz}, 1 \mathrm{H}), 1.95(\mathrm{~s}, 3 \mathrm{H})$.

${ }^{13}$ C NMR (101 MHz, $\left.\mathrm{CDCl}_{3}\right) \delta 171.65,169.76,135.18,134.62,134.17,131.46,129.78,128.73,128.69$, $128.61,128.50,127.08,67.52,52.70,35.55,23.23$.

IR $\left(\mathrm{CDCl}_{3}, \mathrm{~cm}^{-1}\right) v$ 3275, 3064, 2939, 1742, 1653, 1543, 1444, 1373, 1269, 1177, 1134, 1052, 751, 697. HRMS (ASAP) m/z $\left[\mathrm{C}_{18} \mathrm{H}_{19} \mathrm{ClNO}_{3}\right]^{+}\left([\mathrm{M}+\mathrm{H}]^{+}\right)$calculated 332.1053, found 332.1052.

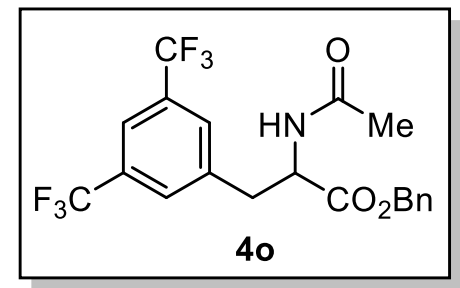

Yield: 52\%, $23 \mathrm{mg}$, white solid.

$\mathbf{R}_{f}=0.46(\mathrm{EA} / \mathrm{Hex} 2: 3)$.

${ }^{1}$ H NMR $\left(400 \mathrm{MHz}, \mathrm{CDCl}_{3}\right) \delta 7.74(\mathrm{~s}, 1 \mathrm{H}), 7.54-7.49(\mathrm{~m}, 2 \mathrm{H}), 7.42-7.33(\mathrm{~m}, 3 \mathrm{H}), 7.33-7.25(\mathrm{~m}, 2 \mathrm{H})$, 6.07 (d, $J=7.2 \mathrm{~Hz}, 1 \mathrm{H}), 5.14$ (s, 2H), 4.93 (ddd, $J=7.2,6.4,5.3 \mathrm{~Hz}, 1 \mathrm{H}), 3.32(\mathrm{dd}, J=13.9,6.3 \mathrm{~Hz}, 1 \mathrm{H})$, $3.21(\mathrm{dd}, J=13.9,5.3 \mathrm{~Hz}, 1 \mathrm{H}), 2.00(\mathrm{~s}, 3 \mathrm{H})$.

${ }^{13}$ C NMR $\left(101 \mathrm{MHz}, \mathrm{CDCl}_{3}\right) \delta 170.93,169.87,138.76,134.58,131.84(\mathrm{q}, J=33.3 \mathrm{~Hz}), 129.72(\mathrm{~d}, J=3.9$ Hz), 129.03, 128.94, 128.63, 123.31 (q, $J=272.7 \mathrm{~Hz}), 121.24$ (p, $J=3.9 \mathrm{~Hz}), 67.95,53.19,37.62,23.13$.

${ }^{19} \mathbf{F}$ NMR $\left(376 \mathrm{MHz}, \mathrm{CDCl}_{3}\right) \delta-62.87(\mathrm{~s})$.

IR $\left(\mathrm{CDCl}_{3}, \mathrm{~cm}^{-1}\right) v 3271,3068,2957,1741,1654,1545,1378,1276,1171,1129,900,700,682$. 
HRMS (ASAP) m/z $\left[\mathrm{C}_{20} \mathrm{H}_{18} \mathrm{~F}_{6} \mathrm{NO}_{3}\right]^{+}\left([\mathrm{M}+\mathrm{H}]^{+}\right)$calculated 434.1191, found 434.1189.

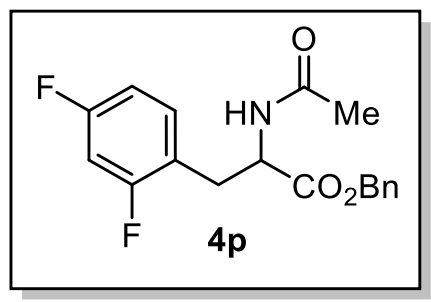

Yield: $65 \%, 22 \mathrm{mg}$, colorless oil.

$\mathbf{R}_{f}=0.28(\mathrm{EA} / \mathrm{Hex} 2: 3)$.

${ }^{1} \mathbf{H}$ NMR $\left(400 \mathrm{MHz}, \mathrm{CDCl}_{3}\right) \delta 7.42-7.28(\mathrm{~m}, 5 \mathrm{H}), 6.97(\mathrm{td}, J=8.6,6.3 \mathrm{~Hz}, 1 \mathrm{H}), 6.78-6.67(\mathrm{~m}, 2 \mathrm{H})$, $6.03(\mathrm{~d}, J=7.9 \mathrm{~Hz}, 1 \mathrm{H}), 5.14(\mathrm{~s}, 2 \mathrm{H}), 4.89$ (dt, $J=7.9,6.0 \mathrm{~Hz}, 1 \mathrm{H}), 3.19$ (ddd, $J=14.1,6.1,1.2 \mathrm{~Hz}, 1 \mathrm{H})$, 3.07 (dd, $J=14.1,5.9 \mathrm{~Hz}, 1 \mathrm{H}), 1.97(\mathrm{~s}, 3 \mathrm{H})$.

${ }^{13}$ C NMR (101 MHz, $\left.\mathrm{CDCl}_{3}\right) \delta 171.41,169.77,162.26(\mathrm{dd}, J=248.4,12.1 \mathrm{~Hz}), 161.42(\mathrm{dd}, J=247.8$, $11.9 \mathrm{~Hz}), 135.05,132.37$ (dd, $J=9.6,6.2 \mathrm{~Hz}), 128.77,128.75,128.70,118.91$ (dd, $J=16.3,3.8 \mathrm{~Hz}), 111.46$ $(\mathrm{dd}, J=21.2,3.7 \mathrm{~Hz}), 103.93(\mathrm{dd}, J=25.7,26.0 \mathrm{~Hz}), 67.66,52.61,31.10,23.18$.

${ }^{19} \mathbf{F ~ N M R}\left(376 \mathrm{MHz}, \mathrm{CDCl}_{3}\right) \delta-111.23(\mathrm{p}, J=7.7 \mathrm{~Hz}),-113.16(\mathrm{q}, J=8.7 \mathrm{~Hz})$.

IR $\left(\mathrm{CDCl}_{3}, \mathrm{~cm}^{-1}\right) v$ 3278, 3066, 3029, 2939, 1740, 1654, 1540, 1504, 1374, 1273, 1174, 1136, 1094, 965, $850,747,697$.

HRMS (ASAP) m/z $\left[\mathrm{C}_{18} \mathrm{H}_{18} \mathrm{~F}_{2} \mathrm{NO}_{3}\right]^{+}\left([\mathrm{M}+\mathrm{H}]^{+}\right)$calculated 334.1255, found 334.1255.

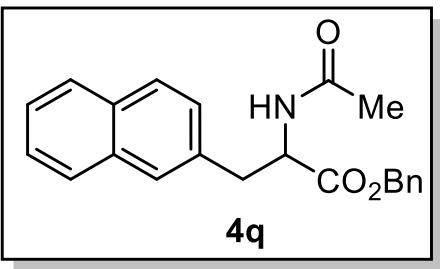

Yield: 52\%, $18 \mathrm{mg}$, white solid.

$\mathbf{R}_{f}=0.26(\mathrm{EA} / \mathrm{Hex} 2: 3)$.

${ }^{1} \mathbf{H}$ NMR $\left(400 \mathrm{MHz}, \mathrm{CDCl}_{3}\right) \delta 8.08(\mathrm{~d}, J=8.6 \mathrm{~Hz}, 1 \mathrm{H}), 7.89-7.80(\mathrm{~m}, 1 \mathrm{H}), 7.73(\mathrm{~d}, J=8.3 \mathrm{~Hz}, 1 \mathrm{H}), 7.55$ - 7.41 (m, 2H), $7.34-7.23$ (m, 4H), $7.20-7.09$ (m, 3H), 5.99 (d, J= 7.8 Hz, 1H), $5.10-4.97$ (m, 3H), $3.65-3.49(\mathrm{~m}, 2 \mathrm{H}), 1.91(\mathrm{~s}, 3 \mathrm{H})$. 
${ }^{13}$ C NMR $\left(101 \mathrm{MHz}, \mathrm{CDCl}_{3}\right) \delta 171.88,169.84,135.03,134.01,132.46,132.37,128.93,128.67,128.58$, $128.49,128.06,127.63,126.42,125.90,125.41,123.72,67.36,53.47,35.22,23.23$.

IR $\left(\mathrm{CDCl}_{3}, \mathrm{~cm}^{-1}\right) v 3277,3062,2953,2251,1739,1652,1539,1511,1372,1176,905,778,728,698$.

HRMS (ASAP) m/z $\left[\mathrm{C}_{22} \mathrm{H}_{22} \mathrm{NO}_{3}\right]^{+}\left([\mathrm{M}+\mathrm{H}]^{+}\right)$calculated 348.1600, found 348.1603.

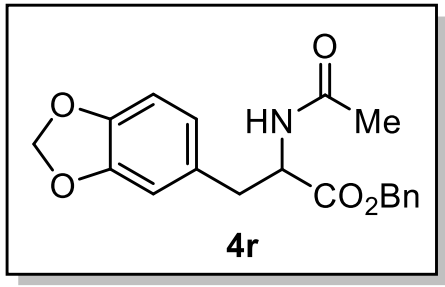

Yield: $65 \%, 22 \mathrm{mg}$, white solid.

$\mathbf{R}_{f}=0.37(\mathrm{EA} / \mathrm{Hex} 1: 1)$.

${ }^{1}$ H NMR (400 MHz, $\left.\mathrm{CDCl}_{3}\right) \delta 7.40$ - $7.34(\mathrm{~m}, 3 \mathrm{H}), 7.34-7.29(\mathrm{~m}, 2 \mathrm{H}), 6.64(\mathrm{~d}, J=7.9 \mathrm{~Hz}, 1 \mathrm{H}), 6.49(\mathrm{~d}$, $J=1.7 \mathrm{~Hz}, 1 \mathrm{H}), 6.42(\mathrm{dd}, J=7.9,1.7 \mathrm{~Hz}, 1 \mathrm{H}), 5.95(\mathrm{~d}, J=8.0 \mathrm{~Hz}, 1 \mathrm{H}), 5.91(\mathrm{~s}, 2 \mathrm{H}), 5.18(\mathrm{~d}, J=12.1 \mathrm{~Hz}$, 1H), $5.12(\mathrm{~d}, J=12.1 \mathrm{~Hz}, 1 \mathrm{H}), 4.87(\mathrm{dt}, J=7.8,5.7 \mathrm{~Hz}, 1 \mathrm{H}), 3.07-2.98(\mathrm{~m}, 2 \mathrm{H}), 1.99(\mathrm{~s}, 3 \mathrm{H})$.

${ }^{13}$ C NMR $\left(101 \mathrm{MHz}, \mathrm{CDCl}_{3}\right) \delta 171.59,169.70,147.87,146.81,135.14,129.40,128.79,128.74,128.71$, $122.53,109.71,108.41,101.09,67.43,53.42,37.68,23.31$.

IR $\left(\mathrm{CDCl}_{3}, \mathrm{~cm}^{-1}\right) v 3281,3065,3034,2927,2893,1738,1654,1537,1501,1489,1443,1371,1246,1191$, 1037, 904, 729, 699.

HRMS (ASAP) m/z $\left[\mathrm{C}_{19} \mathrm{H}_{20} \mathrm{NO}_{5}\right]^{+}\left([\mathrm{M}+\mathrm{H}]^{+}\right)$calculated 342.1341, found 342.1337.

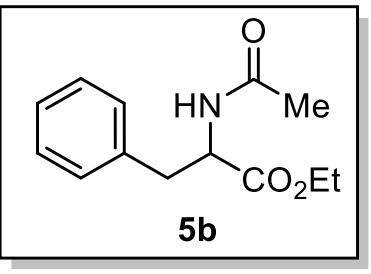

Yield: $68 \%, 16 \mathrm{mg}$, white solid.

$\mathbf{R}_{f}=0.30(\mathrm{EA} / \mathrm{Hex} 2: 3)$. 
${ }^{1}$ H NMR $\left(400 \mathrm{MHz}, \mathrm{CDCl}_{3}\right) \delta 7.33-7.19(\mathrm{~m}, 3 \mathrm{H}), 7.14-7.07(\mathrm{~m}, 2 \mathrm{H}), 5.93(\mathrm{~d}, J=7.8 \mathrm{~Hz}, 1 \mathrm{H}), 4.86(\mathrm{dt}$, $J=7.9,5.8 \mathrm{~Hz}, 1 \mathrm{H}), 4.17(\mathrm{qd}, J=7.2,1.2 \mathrm{~Hz}, 2 \mathrm{H}), 3.19-3.05(\mathrm{~m}, 2 \mathrm{H}), 1.98(\mathrm{~s}, 3 \mathrm{H}), 1.24(\mathrm{t}, J=7.1 \mathrm{~Hz}$, $3 \mathrm{H})$.

${ }^{13} \mathrm{C} \mathrm{NMR}\left(101 \mathrm{MHz}, \mathrm{CDCl}_{3}\right) \delta 171.79,169.72,136.02,129.42,128.62,127.18,61.62,53.26,38.02,23.25$, 14.21.

IR $\left(\mathrm{CDCl}_{3}, \mathrm{~cm}^{-1}\right) v 3283,3063,3030,2982,2932,1737,1656,1542,1374,1212,1028,701$.

HRMS (ASAP) m/z [ $\left.\mathrm{C}_{13} \mathrm{H}_{18} \mathrm{NO}_{3}\right]^{+}\left([\mathrm{M}+\mathrm{H}]^{+}\right)$calculated 236.1287, found 236.1284.

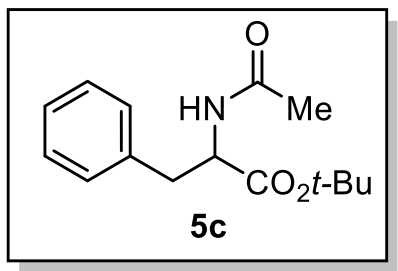

Yield: $63 \%, 17 \mathrm{mg}$, yellowish oil.

$\mathbf{R}_{f}=0.31(\mathrm{EA} / \mathrm{Hex} 2: 3)$.

${ }^{1} \mathbf{H}$ NMR $\left(400 \mathrm{MHz}, \mathrm{CDCl}_{3}\right) \delta 7.35-7.18(\mathrm{~m}, 3 \mathrm{H}), 7.18-7.05(\mathrm{~m}, 2 \mathrm{H}), 5.97(\mathrm{~d}, J=7.7 \mathrm{~Hz}, 1 \mathrm{H}), 4.76(\mathrm{dt}$, $J=7.8,6.0 \mathrm{~Hz}, 1 \mathrm{H}), 3.09(\mathrm{~d}, J=6.0 \mathrm{~Hz}, 2 \mathrm{H}), 1.98(\mathrm{~s}, 3 \mathrm{H}), 1.41(\mathrm{~s}, 9 \mathrm{H})$.

${ }^{13} \mathbf{C}$ NMR $\left(101 \mathrm{MHz}, \mathrm{CDCl}_{3}\right) \delta 170.94,169.64,136.32,129.62,128.49,127.08,82.53,53.65,38.18,28.07$, 23.34 .

IR $\left(\mathrm{CDCl}_{3}, \mathrm{~cm}^{-1}\right)$ v 3285, 3064, 3030, 2978, 2931, 1732, 1655, 1544, 1498, 1369, 1257, 1226, 1154, 755, $738,699$.

HRMS (ESI) m/z [C $\left.{ }_{15} \mathrm{H}_{21} \mathrm{NO}_{3} \mathrm{Na}\right]^{+}\left([\mathrm{M}+\mathrm{H}]^{+}\right)$calculated 286.1419, found 286.1433.

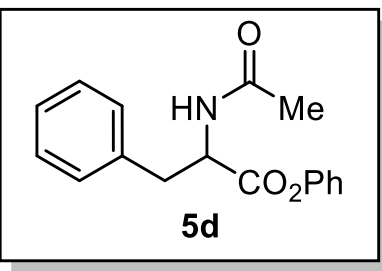

Yield: $65 \%, 18 \mathrm{mg}$, white solid.

$\mathbf{R}_{f}=0.28(\mathrm{EA} / \mathrm{Hex} 2: 3)$. 
${ }^{1}$ H NMR $\left(400 \mathrm{MHz}, \mathrm{CDCl}_{3}\right) \delta 7.41-7.19(\mathrm{~m}, 8 \mathrm{H}), 7.03-6.95(\mathrm{~m}, 2 \mathrm{H}), 6.04(\mathrm{~d}, J=7.8 \mathrm{~Hz}, 1 \mathrm{H}), 5.12(\mathrm{dt}$, $J=7.7,6.0 \mathrm{~Hz}, 1 \mathrm{H}), 3.34-3.20(\mathrm{~m}, 2 \mathrm{H}), 2.01(\mathrm{~s}, 3 \mathrm{H})$.

${ }^{13}$ C NMR $\left(101 \mathrm{MHz}, \mathrm{CDCl}_{3}\right) \delta 170.50,169.89,150.38,135.72,129.63,129.57,128.86,127.49,126.33$, $121.37,53.44,38.08,23.23$.

IR $\left(\mathrm{CDCl}_{3}, \mathrm{~cm}^{-1}\right) v$ 3267, 3033, 2936, 1760, 1653, 1537, 1491, 1372, 1190, 1162, 1121, 908, 734, 699, 494.

HRMS (ESI) m/z [ $\left.\mathrm{C}_{17} \mathrm{H}_{18} \mathrm{NO}_{3}\right]^{+}\left([\mathrm{M}+\mathrm{H}]^{+}\right)$calculated 284.1287, found 284.1281.

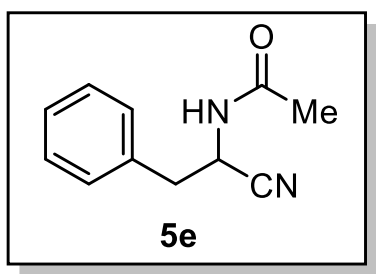

Yield: 70\%, $13 \mathrm{mg}$, white solid.

$\mathbf{R}_{f}=0.27(\mathrm{EA} / \mathrm{Hex} 2: 3)$.

${ }^{1}$ H NMR (400 MHz, $\left.\mathrm{CDCl}_{3}\right) \delta 7.41-7.28(\mathrm{~m}, 3 \mathrm{H}), 7.31-7.24(\mathrm{~m}, 2 \mathrm{H}), 6.17(\mathrm{~d}, J=8.6 \mathrm{~Hz}, 1 \mathrm{H}), 5.14$ (ddd, $J=8.7,7.4,5.8 \mathrm{~Hz}, 1 \mathrm{H}), 3.08(\mathrm{~m}, 2 \mathrm{H}), 1.98(\mathrm{~s}, 3 \mathrm{H})$.

${ }^{13} \mathrm{C}$ NMR $\left(101 \mathrm{MHz}, \mathrm{CDCl}_{3}\right) \delta 169.56,134.03,129.57,129.14,128.09,118.30,41.66,38.88,22.89$.

IR $\left(\mathrm{CDCl}_{3}, \mathrm{~cm}^{-1}\right) v 3270,3032,2932,2856,2244,1954,1658,1531,1497,1372,1289,749,700$.

HRMS (ASAP) m/z $\left[\mathrm{C}_{11} \mathrm{H}_{13} \mathrm{~N}_{2} \mathrm{O}\right]^{+}\left([\mathrm{M}+\mathrm{H}]^{+}\right)$calculated 189.1028, found 189.1029.

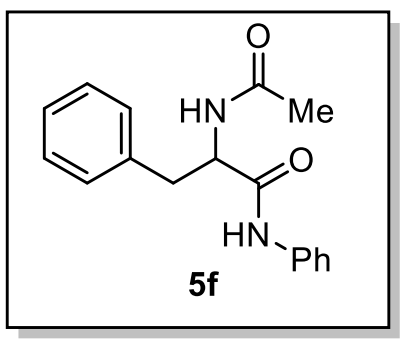

Yield: $71 \%, 20 \mathrm{mg}$, white solid.

$\mathbf{R}_{f}=0.42(\mathrm{EA} / \mathrm{Hex} 2: 3)$. 
${ }^{1}$ H NMR (400 MHz, DMSO- $\left.d_{6}\right) \delta 10.07$ (s, $\left.1 \mathrm{H}\right), 8.27$ (d, $\left.J=8.2 \mathrm{~Hz}, 1 \mathrm{H}\right), 7.57$ (d, $\left.J=7.9 \mathrm{~Hz}, 2 \mathrm{H}\right), 7.34-$ $7.24(\mathrm{~m}, 6 \mathrm{H}), 7.21-7.16(\mathrm{~m}, 1 \mathrm{H}), 7.05(\mathrm{t}, J=7.3 \mathrm{~Hz}, 1 \mathrm{H}), 4.66(\mathrm{td}, J=8.9,5.3 \mathrm{~Hz}, 1 \mathrm{H}), 3.02(\mathrm{dd}, J=$ 13.7, $5.3 \mathrm{~Hz}, 1 \mathrm{H}), 2.84(\mathrm{dd}, J=13.7,9.4 \mathrm{~Hz}, 1 \mathrm{H}), 1.79$ (s, 3H).

${ }^{13}$ C NMR (101 MHz, DMSO- $\left.d_{6}\right) \delta 170.34,169.26,138.81,137.72,129.14,128.68,128.06,126.33,123.37$, $119.34,54.86,37.76,22.39$.

IR (DMSO- $\left.d_{6}, \mathrm{~cm}^{-1}\right) v 3446,2249,2123,1767,1665,1548,1222,1052,1024,1006,820,757,621$.

HRMS (ASAP) m/z $\left[\mathrm{C}_{17} \mathrm{H}_{19} \mathrm{~N}_{2} \mathrm{O}_{2}\right]^{+}\left([\mathrm{M}+\mathrm{H}]^{+}\right)$calculated 283.1447, found 283.1446.

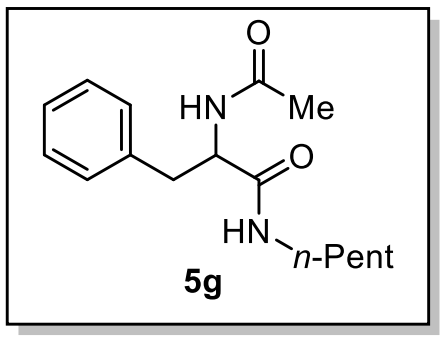

Yield: 59\%, 16 mg, yellow solid.

$\mathbf{R}_{f}=0.29(\mathrm{EA} / \mathrm{Hex} 1: 1)$.

${ }^{1} \mathbf{H}$ NMR $\left(400 \mathrm{MHz}, \mathrm{CDCl}_{3}\right) \delta 7.30-7.24(\mathrm{~m}, 2 \mathrm{H}), 7.27-7.16(\mathrm{~m}, 3 \mathrm{H}), 6.70(\mathrm{~d}, J=8.1 \mathrm{~Hz}, 1 \mathrm{H}), 6.15(\mathrm{t}$, $J=5.7 \mathrm{~Hz}, 1 \mathrm{H}), 4.64(\mathrm{td}, J=8.3,6.4 \mathrm{~Hz}, 1 \mathrm{H}), 3.24-3.11(\mathrm{~m}, 1 \mathrm{H}), 3.10-2.94(\mathrm{~m}, 3 \mathrm{H}), 1.96(\mathrm{~s}, 3 \mathrm{H}), 1.40$ $-1.18(\mathrm{~m}, 4 \mathrm{H}), 1.23-1.06(\mathrm{~m}, 2 \mathrm{H}), 0.85(\mathrm{t}, J=7.3 \mathrm{~Hz}, 3 \mathrm{H})$.

${ }^{13}$ C NMR $\left(101 \mathrm{MHz}, \mathrm{CDCl}_{3}\right) \delta 170.96,170.11,137.01,129.39,128.67,127.00,54.93,39.59,39.03,29.01$, $28.99,23.25,22.38,14.02$.

IR $\left(\mathrm{CDCl}_{3}, \mathrm{~cm}^{-1}\right) v$ 3277, 3085, 3030, 2956, 2929, 2859, 1638, 1552, 1497, 1372, 743, 698.

HRMS (ASAP) m/z $\left[\mathrm{C}_{16} \mathrm{H}_{25} \mathrm{~N}_{2} \mathrm{O}_{2}\right]^{+}\left([\mathrm{M}+\mathrm{H}]^{+}\right)$calculated 277.1916, found 277.1913.

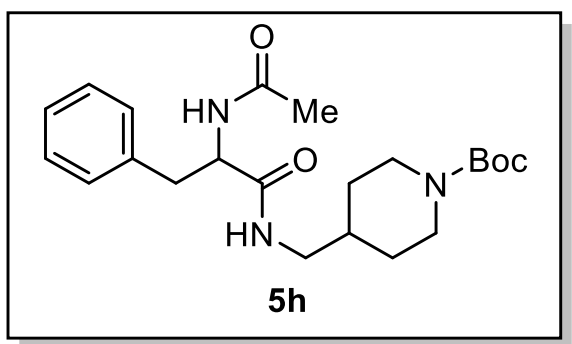


Yield: $74 \%, 30 \mathrm{mg}$, yellow oil.

$\mathbf{R}_{f}=0.22($ EA $100 \%)$

${ }^{1}$ H NMR $\left(400 \mathrm{MHz}, \mathrm{CDCl}_{3}\right) \delta 7.31-7.12(\mathrm{~m}, 5 \mathrm{H}), 6.63(\mathrm{~d}, J=8.1 \mathrm{~Hz}, 1 \mathrm{H}), 6.43(\mathrm{t}, J=5.6 \mathrm{~Hz}, 1 \mathrm{H}), 4.68$ $(\mathrm{td}, J=8.5,6.3 \mathrm{~Hz}, 1 \mathrm{H}), 4.00(\mathrm{~s}, 2 \mathrm{H}), 3.22-3.08(\mathrm{~m}, 1 \mathrm{H}), 3.03(\mathrm{dd}, J=13.4,6.3 \mathrm{~Hz}, 1 \mathrm{H}), 2.96(\mathrm{dd}, J=$ 13.4, 8.7 Hz, 1H), $2.86(\mathrm{~s}, 1 \mathrm{H}), 2.66-2.45(\mathrm{~m}, 2 \mathrm{H}), 1.96$ (s, 3H), $1.43(\mathrm{~s}, 9 \mathrm{H}), 1.41-1.21(\mathrm{~m}, 3 \mathrm{H}), 1.01-$ $0.84(\mathrm{~m}, 2 \mathrm{H})$.

${ }^{13} \mathrm{C}$ NMR $\left(101 \mathrm{MHz}, \mathrm{CDCl}_{3}\right) \delta 171.19,170.17,154.86,136.81,129.37,128.72,127.06,79.52,54.95$, $44.91,43.75,38.98,36.21,29.64,28.56,23.26$.

IR $\left(\mathrm{CDCl}_{3}, \mathrm{~cm}^{-1}\right) v 3281,2972,2923,2849,1686,1640,1547,1421,1365,1277,1226,1168,1141,734$, 698.

HRMS (ESI) $\mathrm{m} / \mathrm{z}\left[\mathrm{C}_{22} \mathrm{H}_{33} \mathrm{~N}_{3} \mathrm{NaO}_{4}\right]^{+}\left([\mathrm{M}+\mathrm{Na}]^{+}\right)$calculated 426.2369, found 426.2367.

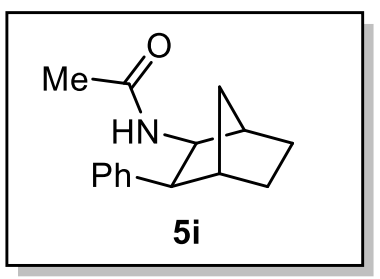

Yield: $61 \%, 14 \mathrm{mg}$, white solid.

$\mathbf{R}_{f}=0.42(\mathrm{EA} / \mathrm{Hex} 2: 3)$.

${ }^{1}$ H NMR $\left(400 \mathrm{MHz}, \mathrm{CDCl}_{3}\right) \delta 7.45-7.35(\mathrm{~m}, 2 \mathrm{H}), 7.32-7.22(\mathrm{~m}, 3 \mathrm{H}), 4.79(\mathrm{~d}, J=8.7 \mathrm{~Hz}, 1 \mathrm{H}), 4.29(\mathrm{t}$, $J=8.6 \mathrm{~Hz}, 1 \mathrm{H}), 3.19(\mathrm{~d}, J=8.6 \mathrm{~Hz}, 1 \mathrm{H}), 2.70(\mathrm{~s}, 1 \mathrm{H}), 2.41-2.35(\mathrm{~m}, 1 \mathrm{H}), 1.84-1.64(\mathrm{~m}, 3 \mathrm{H}), 1.62(\mathrm{~s}$, $3 \mathrm{H}), 1.57-1.38(\mathrm{~m}, 3 \mathrm{H})$.

${ }^{13}$ C NMR $\left(101 \mathrm{MHz}, \mathrm{CDCl}_{3}\right) \delta 169.17,140.13,128.54,128.40,126.47,56.52,52.12,42.61,40.28,35.54$, $30.09,26.39,23.24$.

IR $\left(\mathrm{CDCl}_{3}, \mathrm{~cm}^{-1}\right) v 3293,3061,2955,2872,1646,1549,1496,1451,1373,1290,1154,905,726,700,494$. HRMS (ASAP) $\mathrm{m} / \mathrm{z}\left[\mathrm{C}_{15} \mathrm{H}_{20} \mathrm{NO}\right]^{+}\left([\mathrm{M}+\mathrm{H}]^{+}\right)$calculated 230.1545, found 230.1547. 


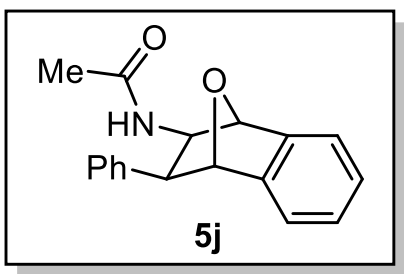

Yield: 89\%, $25 \mathrm{mg}$, white solid.

$\mathbf{R}_{f}=0.42(\mathrm{EA} / \mathrm{Hex} 2: 3)$.

${ }^{1}$ H NMR $\left(400 \mathrm{MHz}, \mathrm{CDCl}_{3}\right) \delta 7.47$ - $7.26(\mathrm{~m}, 7 \mathrm{H}), 7.30$ - $7.20(\mathrm{~m}, 2 \mathrm{H}), 5.56(\mathrm{~s}, 1 \mathrm{H}), 5.31-5.23(\mathrm{~m}, 2 \mathrm{H})$, $4.49(\mathrm{t}, J=8.5 \mathrm{~Hz}, 1 \mathrm{H}), 3.34(\mathrm{~d}, J=7.9 \mathrm{~Hz}, 1 \mathrm{H}), 1.58(\mathrm{~s}, 3 \mathrm{H})$.

${ }^{13}$ C NMR (101 MHz, $\left.\mathrm{CDCl}_{3}\right) \delta 169.86,146.12,143.36,138.74,128.93,128.84,127.64,127.30,127.27$, $120.84,119.13,84.45,84.30,54.24,50.12,22.98$.

IR $\left(\mathrm{CDCl}_{3}, \mathrm{~cm}^{-1}\right) v 3428,3308,3040,3006,1656,1495,1371,904,727,703,648$.

HRMS (ASAP) m/z $\left[\mathrm{C}_{18} \mathrm{H}_{18} \mathrm{NO}_{2}\right]^{+}\left([\mathrm{M}+\mathrm{H}]^{+}\right)$calculated 280.1338, found 280.1337.

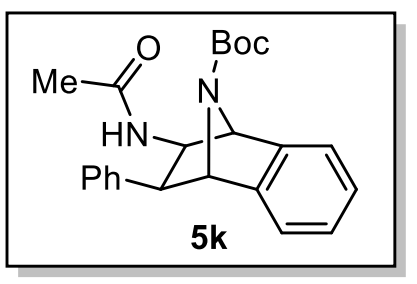

Yield: 55\%, $21 \mathrm{mg}$, white solid.

$\mathbf{R}_{f}=0.26(\mathrm{EA} / \mathrm{Hex} 2: 3)$.

${ }^{1}$ H NMR $\left(400 \mathrm{MHz}, \mathrm{CDCl}_{3}\right) \delta 7.40$ - $7.35(\mathrm{~m}, 1 \mathrm{H}), 7.35$ - $7.28(\mathrm{~m}, 2 \mathrm{H}), 7.28-7.20(\mathrm{~m}, 4 \mathrm{H}), 7.19-7.13$ $(\mathrm{m}, 2 \mathrm{H}), 5.26(\mathrm{~s}, 1 \mathrm{H}), 5.18(\mathrm{~d}, J=9.4 \mathrm{~Hz}, 1 \mathrm{H}), 4.91(\mathrm{~s}, 1 \mathrm{H}), 4.46-4.29(\mathrm{~m}, 1 \mathrm{H}), 3.20(\mathrm{~d}, J=8.1 \mathrm{~Hz}, 1 \mathrm{H})$, $1.51(\mathrm{~s}, 3 \mathrm{H}), 1.34(\mathrm{~s}, 9 \mathrm{H})$.

${ }^{13}$ C NMR $\left(126 \mathrm{MHz}, \mathrm{CDCl}_{3}\right) \delta 169.62,156.59,146.57,143.71,138.95,128.83,128.68,127.54,127.29$, $127.16,121.79,120.04,99.74,81.11,67.68,66.46,53.64,50.12,28.44,22.96$. (Measured at $60{ }^{\circ} \mathrm{C}$. )

IR $\left(\mathrm{CDCl}_{3}, \mathrm{~cm}^{-1}\right) v 3315,3028,2979,2927,1698,1667,1367,1336,1278,1255,1158,909,729$.

HRMS (ESI) $\mathrm{m} / \mathrm{z}\left[\mathrm{C}_{23} \mathrm{H}_{26} \mathrm{~N}_{2} \mathrm{NaO}_{3}\right]^{+}\left([\mathrm{M}+\mathrm{Na}]^{+}\right)$calculated 401.1841, found 401.1851. 


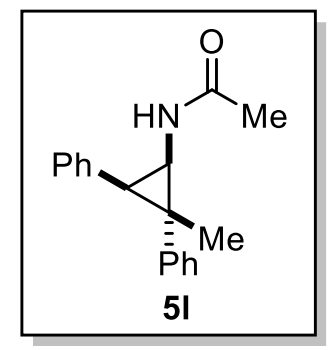

Yield: $43 \%, 11 \mathrm{mg}$, colorless oil.

$\mathbf{R}_{f}=0.37(\mathrm{EA} / \mathrm{Hex} 2: 3)$.

(major diastereomer) ${ }^{1} \mathbf{H}$ NMR $\left(400 \mathrm{MHz}, \mathrm{CDCl}_{3}\right) \delta 7.64-7.58(\mathrm{~m}, 2 \mathrm{H}), 7.48-7.42(\mathrm{~m}, 2 \mathrm{H}), 7.42-7.32$ (m, 5H), $7.32-7.25(\mathrm{~m}, 1 \mathrm{H}), 5.37-5.32(\mathrm{~m}, 1 \mathrm{H}), 3.50(\mathrm{dd}, J=8.6,5.0 \mathrm{~Hz}, 1 \mathrm{H}), 2.69$ (dd, $J=8.6,1.2 \mathrm{~Hz}$, 1H), $2.02(\mathrm{~s}, 3 \mathrm{H}), 1.27(\mathrm{~s}, 3 \mathrm{H})$.

(major diastereomer) ${ }^{13} \mathbf{C}$ NMR $\left(101 \mathrm{MHz}, \mathrm{CDCl}_{3}\right) \delta 171.48,146.83,134.92,130.58,129.25,128.74$, $128.57,127.14,126.65,37.19,30.92,30.24,23.36,18.07$.

IR $\left(\mathrm{CDCl}_{3}, \mathrm{~cm}^{-1}\right) v 3303,3056,3024,2927,1651,1601,1495,1444,1370,1268,907,763,732,700$.

HRMS (ASAP) m/z [C $\left.{ }_{18} \mathrm{H}_{20} \mathrm{NO}\right]^{+}\left([\mathrm{M}+\mathrm{H}]^{+}\right)$calculated 266.1545, found 266.1544.

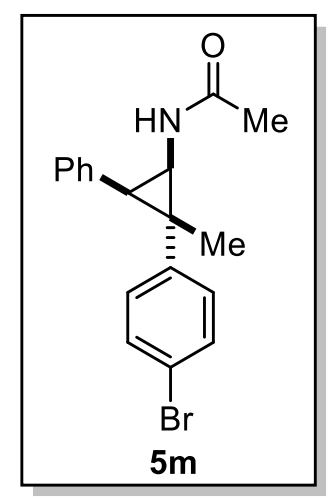

Yield: $38 \%, 13 \mathrm{mg}$, white solid.

$\mathbf{R}_{f}=0.32(\mathrm{EA} / \operatorname{Hex} 2: 3)$.

(major diastereomer) ${ }^{1} \mathbf{H}$ NMR $\left(400 \mathrm{MHz}, \mathrm{CDCl}_{3}\right) \delta 7.54-7.42(\mathrm{~m}, 6 \mathrm{H}), 7.39-7.30(\mathrm{~m}, 3 \mathrm{H}), 5.34(\mathrm{~s}, 1 \mathrm{H})$, $3.41(\mathrm{dd}, J=8.7,4.7 \mathrm{~Hz}, 1 \mathrm{H}), 2.65(\mathrm{dd}, J=8.7,1.1 \mathrm{~Hz}, 1 \mathrm{H}), 2.01(\mathrm{~s}, 3 \mathrm{H}), 1.23$ (s, 3H).

(major diastereomer) ${ }^{13} \mathbf{C}$ NMR (101 MHz, $\left.\mathrm{CDCl}_{3}\right) \delta 171.57,145.93,134.53,131.83,130.53,130.46$, $129.37,127.32,120.50,37.15,30.69,30.17,23.32,17.94$. 
IR $\left(\mathrm{CDCl}_{3}, \mathrm{~cm}^{-1}\right) v$ 3302, 3054, 3026, 2931, 2979, 1653, 1492, 1444, 1369, 1090, 1009, 825, 734, 702, 532.

HRMS (ASAP) m/z [C $\left.{ }_{18} \mathrm{H}_{19} \mathrm{BrNO}\right]^{+}\left([\mathrm{M}+\mathrm{H}]^{+}\right)$calculated 344.0650, found 344.0644.

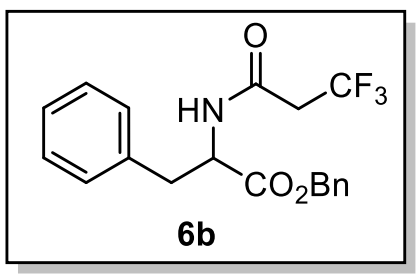

Yield: 73\%, $27 \mathrm{mg}$, white solid.

$\mathbf{R}_{f}=0.30(\mathrm{EA} / \mathrm{Hex} 1: 4)$.

${ }^{1} \mathbf{H}$ NMR $\left(400 \mathrm{MHz}, \mathrm{CDCl}_{3}\right) \delta 7.42-7.35(\mathrm{~m}, 3 \mathrm{H}), 7.34-7.28(\mathrm{~m}, 2 \mathrm{H}), 7.24-7.17$ (m, 3H), $7.01-6.92$ $(\mathrm{m}, 2 \mathrm{H}), 6.19(\mathrm{~d}, J=7.6 \mathrm{~Hz}, 1 \mathrm{H}), 5.20(\mathrm{~d}, J=12.1 \mathrm{~Hz}, 1 \mathrm{H}), 5.14(\mathrm{~d}, J=12.1 \mathrm{~Hz}, 1 \mathrm{H}), 4.94(\mathrm{dt}, J=7.7,5.6$ $\mathrm{Hz}, 1 \mathrm{H}), 3.24-3.09$ (m, 2H), 3.04 (q, $J=10.5 \mathrm{~Hz}, 2 \mathrm{H})$.

${ }^{13}$ C NMR (101 MHz, $\left.\mathrm{CDCl}_{3}\right) \delta 170.94,162.13(\mathrm{q}, J=3.6 \mathrm{~Hz}), 135.23,134.94,129.44,128.85,128.84$, 128.77, 127.42, 123.97 (q, $J=276.9 \mathrm{~Hz}), 67.74,53.59,41.69$ (q, $J=29.7 \mathrm{~Hz}$ ), 37.68. (Two aromatic peaks overlapped)

${ }^{19}$ F NMR $\left(376 \mathrm{MHz}, \mathrm{CDCl}_{3}\right) \delta-62.86(\mathrm{t}, J=10.5 \mathrm{~Hz})$

IR $\left(\mathrm{CDCl}_{3}, \mathrm{~cm}^{-1}\right) v 3313,3066,3032,2957,1740,1664,1542,1388,1261,1240,1213,1191,1136,905$ $733,699$.

HRMS (ASAP) m/z $\left[\mathrm{C}_{19} \mathrm{H}_{19} \mathrm{~F}_{3} \mathrm{NO}_{3}\right]^{+}\left([\mathrm{M}+\mathrm{H}]^{+}\right)$calculated 366.1317, found 366.1319.

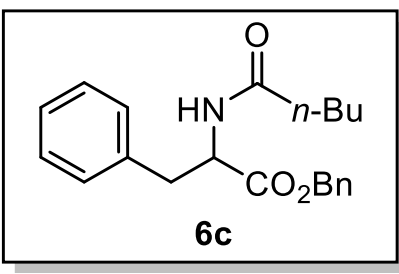

Yield: 53\%, $18 \mathrm{mg}$, white solid.

$\mathbf{R}_{f}=0.23(\mathrm{EA} / \mathrm{Hex} 1: 4)$.

${ }^{1}$ H NMR $\left(400 \mathrm{MHz}, \mathrm{CDCl}_{3}\right) \delta 7.42-7.30(\mathrm{~m}, 3 \mathrm{H}), 7.33$ - 7.27 (m, 2H), $7.24-7.19$ (m, 3H), 7.03 - 6.96 $(\mathrm{m}, 2 \mathrm{H}), 5.87(\mathrm{~d}, J=7.8 \mathrm{~Hz}, 1 \mathrm{H}), 5.17(\mathrm{~d}, J=12.2 \mathrm{~Hz}, 1 \mathrm{H}), 5.11(\mathrm{~d}, J=12.1 \mathrm{~Hz}, 1 \mathrm{H}), 4.95(\mathrm{dt}, J=7.9,5.8$ 
$\mathrm{Hz}, 1 \mathrm{H}), 3.19-3.04(\mathrm{~m}, 2 \mathrm{H}), 2.20-2.12(\mathrm{~m}, 2 \mathrm{H}), 1.62-1.50(\mathrm{~m}, 2 \mathrm{H}), 1.35-1.24(\mathrm{~m}, 2 \mathrm{H}), 0.88(\mathrm{t}, J=$ $7.3 \mathrm{~Hz}, 3 \mathrm{H})$.

${ }^{13}$ C NMR $\left(101 \mathrm{MHz}, \mathrm{CDCl}_{3}\right) \delta 172.76,171.72,135.89,135.20,129.45,128.75,128.72,128.68,128.65$, $127.17,67.39,53.04,38.01,36.40,27.73,22.42,13.88$.

IR $\left(\mathrm{CDCl}_{3}, \mathrm{~cm}^{-1}\right) v 3288,3063,3031,2956,2930,2869,1740,1648,1537,1497,1454,1210,1174,742$, 697.

HRMS (ASAP) m/z $\left[\mathrm{C}_{21} \mathrm{H}_{26} \mathrm{NO}_{3}\right]^{+}\left([\mathrm{M}+\mathrm{H}]^{+}\right)$calculated 340.1913, found 340.1908.

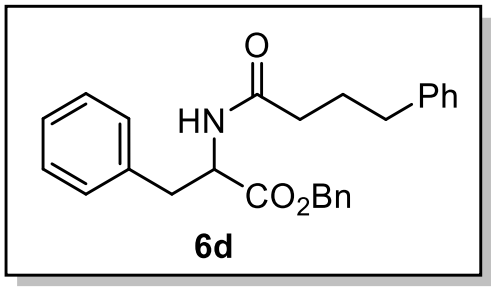

Yield: 54\%, $22 \mathrm{mg}$, white solid.

$\mathbf{R}_{f}=0.25(\mathrm{EA} / \mathrm{Hex} 1: 4)$.

${ }^{1} \mathbf{H}$ NMR $\left(400 \mathrm{MHz}, \mathrm{CDCl}_{3}\right) \delta 7.42-7.08(\mathrm{~m}, 13 \mathrm{H}), 7.04-6.95(\mathrm{~m}, 2 \mathrm{H}), 5.86(\mathrm{~d}, J=7.9 \mathrm{~Hz}, 1 \mathrm{H}), 5.17$ $(\mathrm{d}, J=12.1 \mathrm{~Hz}, 1 \mathrm{H}), 5.11(\mathrm{~d}, J=12.1 \mathrm{~Hz}, 1 \mathrm{H}), 4.94(\mathrm{dt}, J=7.9,5.9 \mathrm{~Hz}, 1 \mathrm{H}), 3.19-3.03(\mathrm{~m}, 2 \mathrm{H}), 2.59$ (t, $J=7.5 \mathrm{~Hz}, 2 \mathrm{H}), 2.16(\mathrm{dd}, J=7.9,6.6 \mathrm{~Hz}, 2 \mathrm{H}), 1.92(\mathrm{p}, J=7.6 \mathrm{~Hz}, 2 \mathrm{H})$.

${ }^{13}$ C NMR (101 MHz, $\left.\mathrm{CDCl}_{3}\right) \delta 172.37,171.67,141.50,135.84,135.17,129.41,128.74,128.71,128.67$, $128.59,128.49,127.19,126.06,67.40,53.07,37.97,35.70,35.14,27.01$. (Two aromatic peaks overlapped) IR $\left(\mathrm{CDCl}_{3}, \mathrm{~cm}^{-1}\right) v 3290,3062,3028,2930,2859,1738,1648,1534,1496,1453,1175,907,734,696$. HRMS (ASAP) m/z $\left[\mathrm{C}_{26} \mathrm{H}_{28} \mathrm{NO}_{3}\right]^{+}\left([\mathrm{M}+\mathrm{H}]^{+}\right)$calculated 402.2069, found 402.2067.

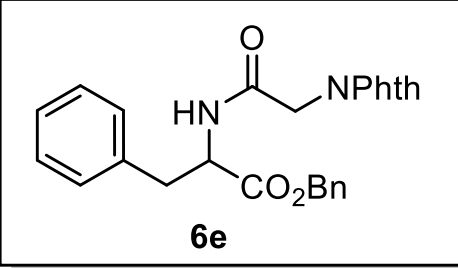

Yield: 56\%, $25 \mathrm{mg}$, white solid. 
$\mathbf{R}_{f}=0.44(\mathrm{EA} / \mathrm{Hex} 2: 3)$.

${ }^{1} \mathbf{H}$ NMR $\left(500 \mathrm{MHz}, \mathrm{CDCl}_{3}\right) \delta 7.95$ - $7.85(\mathrm{~m}, 2 \mathrm{H}), 7.79$ - $7.73(\mathrm{~m}, 2 \mathrm{H}), 7.41$ - $7.32(\mathrm{~m}, 3 \mathrm{H}), 7.31$ - 7.27 (m, 2H), $7.19-7.13(\mathrm{~m}, 3 \mathrm{H}), 7.03-6.90(\mathrm{~m}, 2 \mathrm{H}), 6.24(\mathrm{~d}, J=7.6 \mathrm{~Hz}, 1 \mathrm{H}), 5.18(\mathrm{~d}, J=12.1 \mathrm{~Hz}, 1 \mathrm{H}), 5.12$ $(\mathrm{d}, J=12.0 \mathrm{~Hz}, 1 \mathrm{H}), 4.91(\mathrm{dt}, J=7.8,5.5 \mathrm{~Hz}, 1 \mathrm{H}), 4.38(\mathrm{~d}, J=16.1 \mathrm{~Hz}, 1 \mathrm{H}), 4.30(\mathrm{~d}, J=16.1 \mathrm{~Hz}, 1 \mathrm{H})$, $3.18-3.07(\mathrm{~m}, 2 \mathrm{H})$.

${ }^{13}$ C NMR $\left(126 \mathrm{MHz}, \mathrm{CDCl}_{3}\right) \delta 171.00,167.76,165.66,135.39,134.99,134.38,132.13,129.58,128.84$, $128.80,128.79,128.68,127.24,123.80,67.62,53.53,40.80,37.69$.

IR $\left(\mathrm{CDCl}_{3}, \mathrm{~cm}^{-1}\right) v 3346,3066,3033,2947,1774,1716,1419,1393,1192,954,904,730,716,701$.

HRMS (ASAP) m/z $\left[\mathrm{C}_{26} \mathrm{H}_{23} \mathrm{~N}_{2} \mathrm{O}_{5}\right]^{+}\left([\mathrm{M}+\mathrm{H}]^{+}\right)$calculated 443.1607, found 4431608.

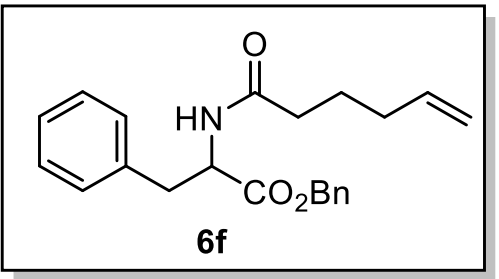

Yield: $61 \%, 21 \mathrm{mg}$, colorless oil.

$\mathbf{R}_{f}=0.22(\mathrm{EA} / \mathrm{Hex} \mathrm{1:4)}$.

${ }^{1} \mathbf{H}$ NMR $\left(400 \mathrm{MHz}, \mathrm{CDCl}_{3}\right) \delta 7.42-7.33(\mathrm{~m}, 3 \mathrm{H}), 7.33-7.28(\mathrm{~m}, 2 \mathrm{H}), 7.24-7.19(\mathrm{~m}, 3 \mathrm{H}), 7.03-6.96$ $(\mathrm{m}, 2 \mathrm{H}), 5.86(\mathrm{~d}, J=7.9 \mathrm{~Hz}, 1 \mathrm{H}), 5.75(\mathrm{ddt}, J=17.0,10.2,6.7 \mathrm{~Hz}, 1 \mathrm{H}), 5.18(\mathrm{~d}, J=12.1 \mathrm{~Hz}, 1 \mathrm{H}), 5.12(\mathrm{~d}$, $J=12.1 \mathrm{~Hz}, 1 \mathrm{H}), 5.02-4.92(\mathrm{~m}, 3 \mathrm{H}), 3.20-3.05(\mathrm{~m}, 2 \mathrm{H}), 2.19-2.15(\mathrm{~m}, 2 \mathrm{H}), 2.07-2.02(\mathrm{~m}, 2 \mathrm{H}), 1.76$ $-1.66(\mathrm{~m}, 2 \mathrm{H})$.

${ }^{13}$ C NMR (101 MHz, $\left.\mathrm{CDCl}_{3}\right) \delta 172.45,171.68,137.90,135.86,135.19,129.44,128.76,128.73,128.69$, 128.67, 127.20, 115.48, 67.41, 53.06, 38.00, 35.76, 33.11, 24.64.

IR $\left(\mathrm{CDCl}_{3}, \mathrm{~cm}^{-1}\right) v$ 3293, 3064, 3031, 2931, 1742, 1648, 1539, 1498, 1454, 1175, 744, 698.

HRMS (ASAP) m/z $\left[\mathrm{C}_{22} \mathrm{H}_{26} \mathrm{NO}_{3}\right]^{+}\left([\mathrm{M}+\mathrm{H}]^{+}\right)$calculated 352.1913, found 352.1911. 


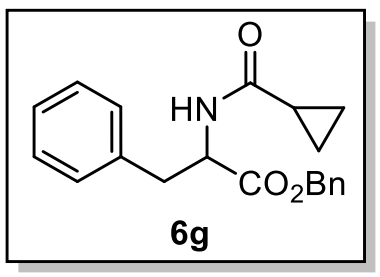

Yield: 53\%, $17 \mathrm{mg}$, white solid.

$\mathbf{R}_{f}=0.27(\mathrm{EA} / \mathrm{Hex} 1: 4)$.

${ }^{1}$ H NMR (400 MHz, $\left.\mathrm{CDCl}_{3}\right) \delta 7.40$ - $7.34(\mathrm{~m}, 3 \mathrm{H}), 7.32$ - 7.28 (m, 2H), 7.25 - 7.19 (m, 3H), 7.07 - 6.98 (m, 2H), $6.10(\mathrm{~d}, J=7.8 \mathrm{~Hz}, 1 \mathrm{H}), 5.18(\mathrm{~d}, J=12.2 \mathrm{~Hz}, 1 \mathrm{H}), 5.12$ (d, $J=12.1 \mathrm{~Hz}, 1 \mathrm{H}), 4.95$ (dt, $J=7.9,5.8$ $\mathrm{Hz}, 1 \mathrm{H}), 3.18-3.07(\mathrm{~m}, 2 \mathrm{H}), 1.36(\mathrm{tt}, J=7.9,4.6 \mathrm{~Hz}, 1 \mathrm{H}), 1.02-0.91(\mathrm{~m}, 2 \mathrm{H}), 0.79-0.68(\mathrm{~m}, 2 \mathrm{H})$.

${ }^{13} \mathrm{C}$ NMR $\left(101 \mathrm{MHz}, \mathrm{CDCl}_{3}\right) \delta 173.24,171.74,135.92,135.21,129.51,128.74,128.71,128.66,128.64$, $127.15,67.35,53.34,38.10,14.75,7.52,7.45$.

IR $\left(\mathrm{CDCl}_{3}, \mathrm{~cm}^{-1}\right) v 3302,3063,3029,3006,2941,2838,1739,1650,1599,1498,1455,1203,1150,1066$ $732,699$.

HRMS (ASAP) m/z [ $\left[\mathrm{C}_{20} \mathrm{H}_{22} \mathrm{NO}_{3}\right]^{+}\left([\mathrm{M}+\mathrm{H}]^{+}\right)$calculated 324.1600, found 324.1601.

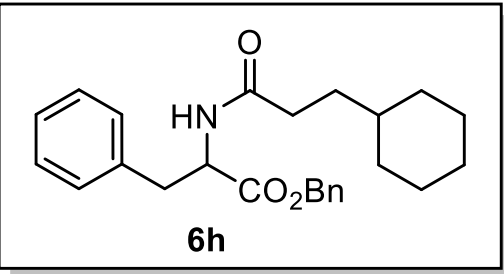

Yield: 57\%, $22 \mathrm{mg}$, colorless oil.

$\mathbf{R}_{f}=0.33(\mathrm{EA} / \mathrm{Hex} \mathrm{1:4)}$.

${ }^{1} \mathbf{H}$ NMR $\left(400 \mathrm{MHz}, \mathrm{CDCl}_{3}\right) \delta 7.44$ - $7.31(\mathrm{~m}, 3 \mathrm{H}), 7.34-7.28(\mathrm{~m}, 2 \mathrm{H}), 7.28$ - $7.18(\mathrm{~m}, 3 \mathrm{H}), 7.04-6.95$ $(\mathrm{m}, 2 \mathrm{H}), 5.86(\mathrm{~d}, J=7.8 \mathrm{~Hz}, 1 \mathrm{H}), 5.18(\mathrm{~d}, J=12.1 \mathrm{~Hz}, 1 \mathrm{H}), 5.12(\mathrm{~d}, J=12.1 \mathrm{~Hz}, 1 \mathrm{H}), 4.95(\mathrm{dt}, J=7.9,5.8$ $\mathrm{Hz}, 1 \mathrm{H}), 3.19-3.05(\mathrm{~m}, 2 \mathrm{H}), 2.24-2.12(\mathrm{~m}, 2 \mathrm{H}), 1.74-1.59(\mathrm{~m}, 5 \mathrm{H}), 1.54-1.42(\mathrm{~m}, 2 \mathrm{H}), 1.28-1.05$ $(\mathrm{m}, 4 \mathrm{H}), 0.94-0.79(\mathrm{~m}, 2 \mathrm{H})$.

${ }^{13}$ C NMR (101 MHz, $\left.\mathrm{CDCl}_{3}\right) \delta 173.03,171.73,135.89,135.21,129.46,128.76,128.73,128.68,128.64$, $127.18,67.39,53.05,38.00,37.34,34.16,33.17,33.11,33.03,26.65,26.33$.

IR $\left(\mathrm{CDCl}_{3}, \mathrm{~cm}^{-1}\right) v 3298,3070,3029,2921,2850,1740,1648,1536,1498,1451,1174,905,730,698$. 
HRMS (ASAP) m/z $\left[\mathrm{C}_{25} \mathrm{H}_{32} \mathrm{NO}_{3}\right]^{+}\left([\mathrm{M}+\mathrm{H}]^{+}\right)$calculated 394.2387, found 394.2384. 


\section{Mechanistic studies}

Possible aziridine intermediate

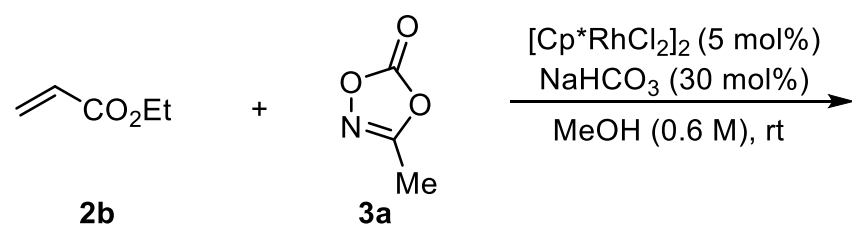

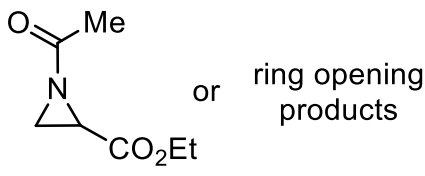

Not observed

$\left[\mathrm{Cp}^{*} \mathrm{RhCl}_{2}\right]_{2}(0.0050 \mathrm{mmol}, 5 \mathrm{~mol} \%)$ and sodium bicarbonate $(0.03 \mathrm{mmol}, 30 \mathrm{~mol} \%)$ were measured in a 0.5-dram vial with a magnetic stir bar. In a separate vial, dioxazolone 3a ( $0.1 \mathrm{mmol}, 1$ equiv) and ethyl acrylate $\mathbf{2 b}$ ( $0.3 \mathrm{mmol}, 3$ equiv) were dissolved in $0.10 \mathrm{~mL}$ of $\mathrm{MeOH}$ and transferred to the first vial. It was rinsed with an additional $0.07 \mathrm{~mL}$ of $\mathrm{MeOH}$ and transferred again to the first vial. The reaction mixture was then stirred at room temperature for 16 hours. After the completion of the reaction, the solvent was removed by rotary evaporator. Ethyl acetate was added to the residue and filtered through a short pad of celite and washed with ethyl acetate three times. The filtrate was concentrated by rotary evaporator and a crude ${ }^{1} \mathrm{H}$ NMR spectrum was collected with 1,3,5-trimethoxybenzene as the internal standard. As a result, neither aziridine nor ring-opening products by methanol were observed. This result suggests $\mathrm{Rh}(\mathrm{III})$-catalyzed aziridination and subsequent ring-opening by phenylboronic acid is unlikely for the mechanism of the carboamination. 


\section{Reversibility of $\beta$-hydride elimination}
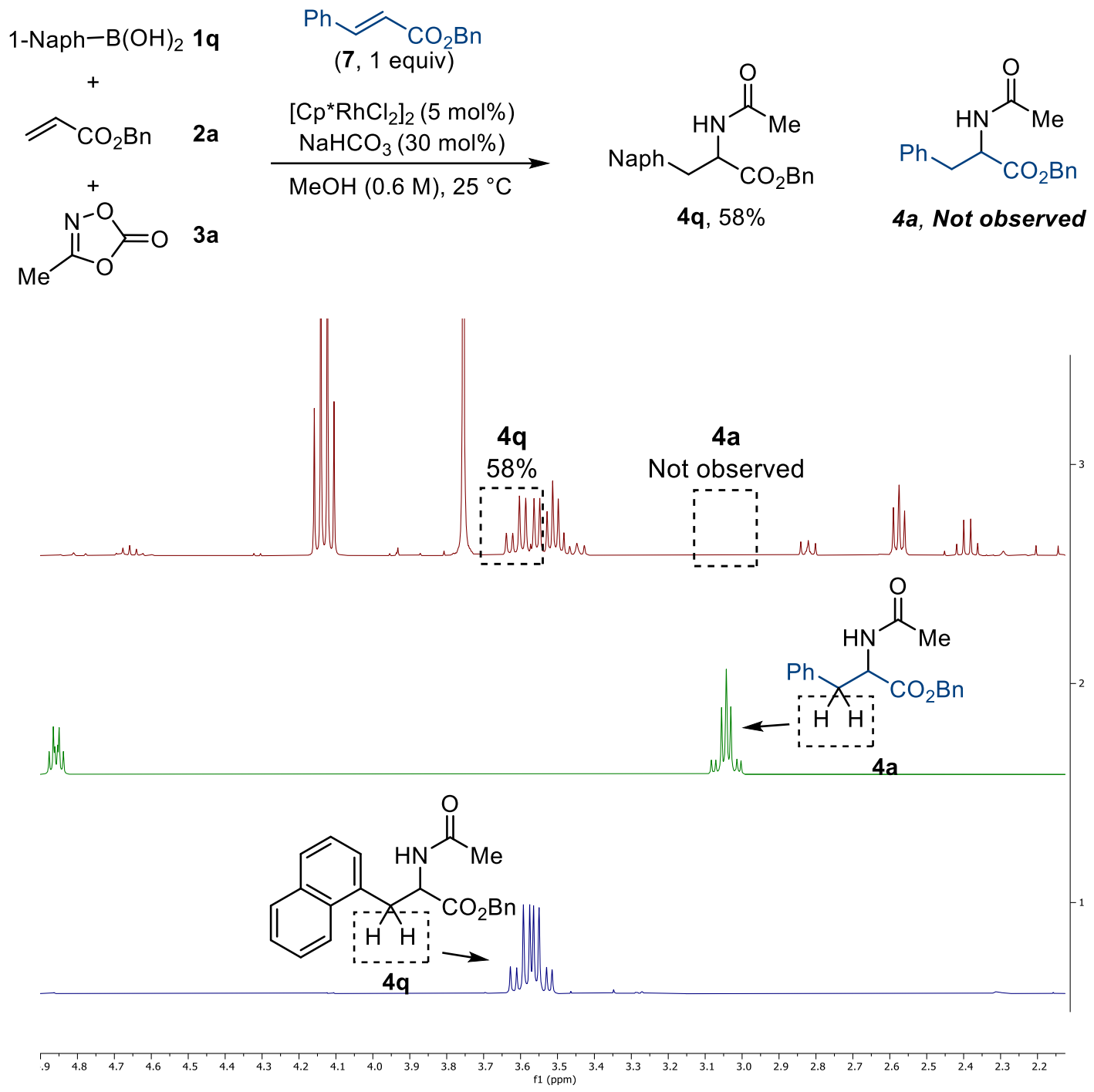

Benzyl cinnamate 7 was prepared according to literature procedures. ${ }^{9}\left[\mathrm{Cp}^{*} \mathrm{RhCl}_{2}\right]_{2}(0.0050 \mathrm{mmol}, 5 \mathrm{~mol} \%)$ and sodium bicarbonate $(0.03 \mathrm{mmol}, 30 \mathrm{~mol} \%)$, and naphthalene-1-boronic acid 1q ( $0.25 \mathrm{mmol}, 2.5$ equiv) were measured in a 0.5-dram vial with a magnetic stir bar. In a separate vial, benzyl cinnamate 7 ( $0.1 \mathrm{mmol}$, 1 equiv) was dissolved $0.10 \mathrm{~mL}$ of $\mathrm{MeOH}$ and transferred to the first vial. It was rinsed with an additional $0.07 \mathrm{~mL}$ of $\mathrm{MeOH}$ and transferred again to the first vial. Sequentially benzyl acrylate $\mathbf{2 a}(0.3 \mathrm{mmol}, 3$ equiv) and dioxazolone 3a $(0.1 \mathrm{mmol}, 1$ equiv) were added to the reaction mixture using a micropipette. The reaction mixture was then stirred at room temperature for 16 hours. After the completion of the reaction, the solvent was removed by a rotary evaporator. Ethyl acetate was added to the residue and filtered through a short pad of celite and washed with ethyl acetate three times. The filtrate was concentrated by rotary 
evaporator and a crude ${ }^{1} \mathrm{H}$ NMR spectrum was collected with 1,3,5-trimethoxybenzene as the internal standard).

\section{Reversibility of proto-demetalation step}
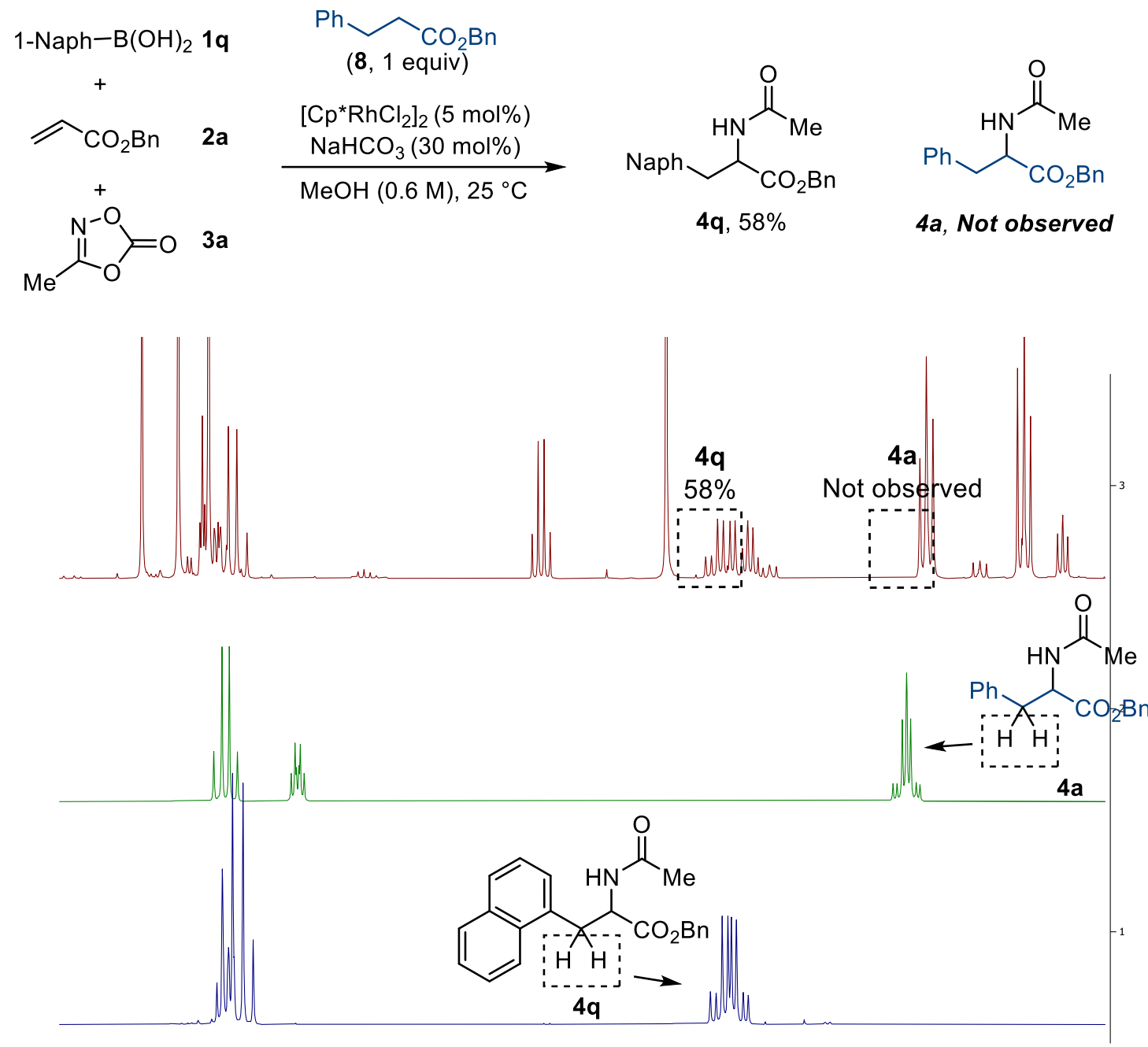

$\begin{array}{lllllllllllllllllllllllllllllllllllllllllll}5.5 & 5.4 & 5.3 & 5.2 & 5.1 & 5.0 & 4.9 & 4.8 & 4.7 & 4.6 & 4.5 & 4.4 & 4.3 & 4.2 & 4.1 & 4.0 & 3.9 & 3.8 & 3.7 & 3.6 & 3.5 & 3.4 & 3.3 & 3.2 & 3.1 & 3.0 & 2.9 & 2.8 & 2.7 & 2.6 & 2.5\end{array}$

Benzyl 3-phenylpropanoate 8 was prepared according to literature procedures. ${ }^{10}\left[\mathrm{Cp}^{*} \mathrm{RhCl}_{2}\right]_{2}(0.0050 \mathrm{mmol}$, $5 \mathrm{~mol} \%)$ and sodium bicarbonate $(0.03 \mathrm{mmol}, 30 \mathrm{~mol} \%)$, and naphthalene-1-boronic acid $1 \mathrm{q}(0.25 \mathrm{mmol}$, 2.5 equiv) were measured in a 0.5-dram vial with a magnetic stir bar. In a separate vial, Benzyl 3phenylpropanoate $\mathbf{8}(0.1 \mathrm{mmol}, 1$ equiv) were dissolved $0.10 \mathrm{~mL}$ of $\mathrm{MeOH}$ and transferred to the first vial. It was rinsed with an additional $0.7 \mathrm{~mL}$ of $\mathrm{MeOH}$ and transferred again to the first vial. Sequentially benzyl acrylate $\mathbf{2 a}(0.3 \mathrm{mmol}, 3$ equiv) and dioxazolone $\mathbf{3 a}$ ( $0.1 \mathrm{mmol}, 1$ equiv) were added to the reaction mixture 
using a micropipette. The reaction mixture was then stirred at room temperature for 16 hours. After the completion of the reaction, the solvent was removed by a rotary evaporator. Ethyl acetate was added to the residue and filtered through a short pad of celite and washed with ethyl acetate three times. The filtrate was concentrated by rotary evaporator and a crude ${ }^{1} \mathrm{H}$ NMR spectrum was collected with 1,3,5trimethoxybenzene as the internal standard. While both reactions give the desired product (4q) from 1naphthyl boronic acid (2q) in good yield, carboamination products (4a) from these side products (7 or $\mathbf{8})$ are not observed. This experiment suggests $\mathbf{7}$ and $\mathbf{8}$ are off-cycle side products that do not re-enter the catalytic cycle.
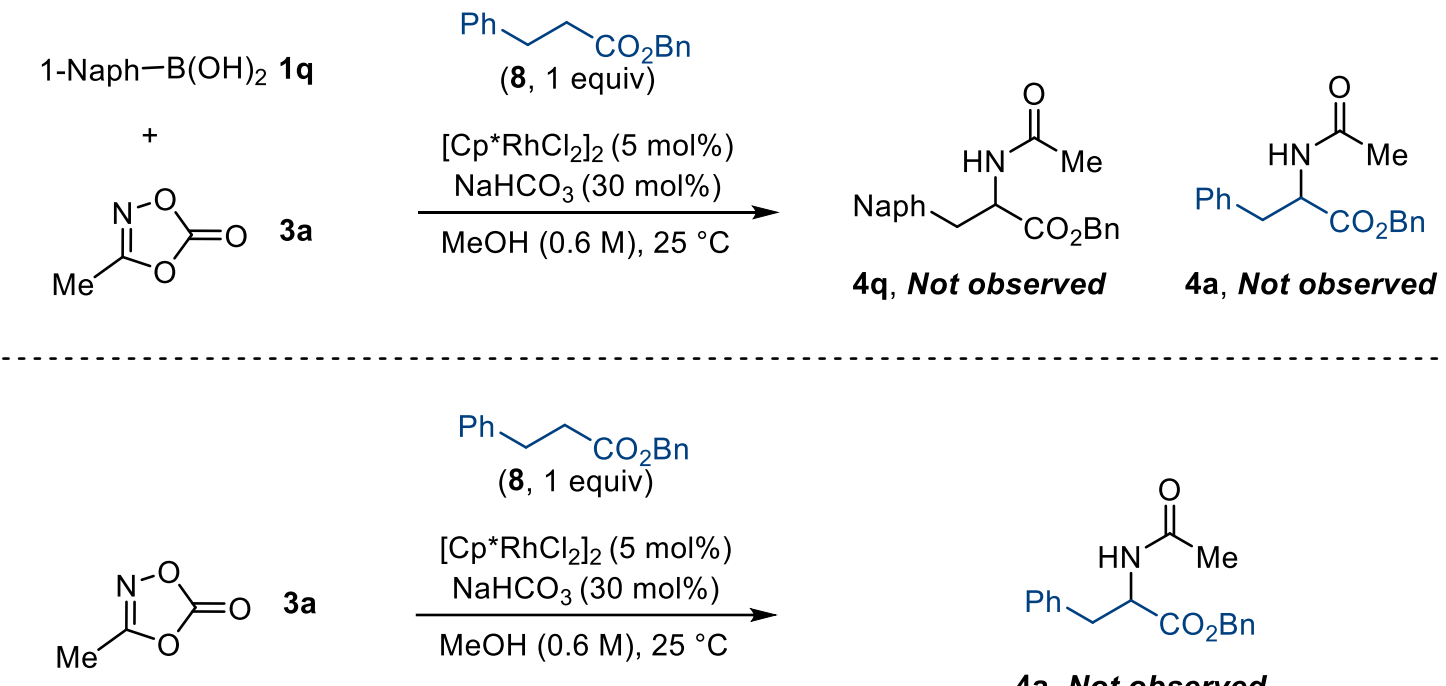

\section{4a, Not observed}

The same experiments were conducted without benzyl acrylate (2a) and without both naphthalene-1boronic acid (1q) and benzyl acrylate (2a). As a result, carboamination products are not observed after the reaction, which again supports benzyl 3-phenylpropanoate $(\mathbf{8})$ as an off-cycle side product. 


\section{Reaction progress monitoring}

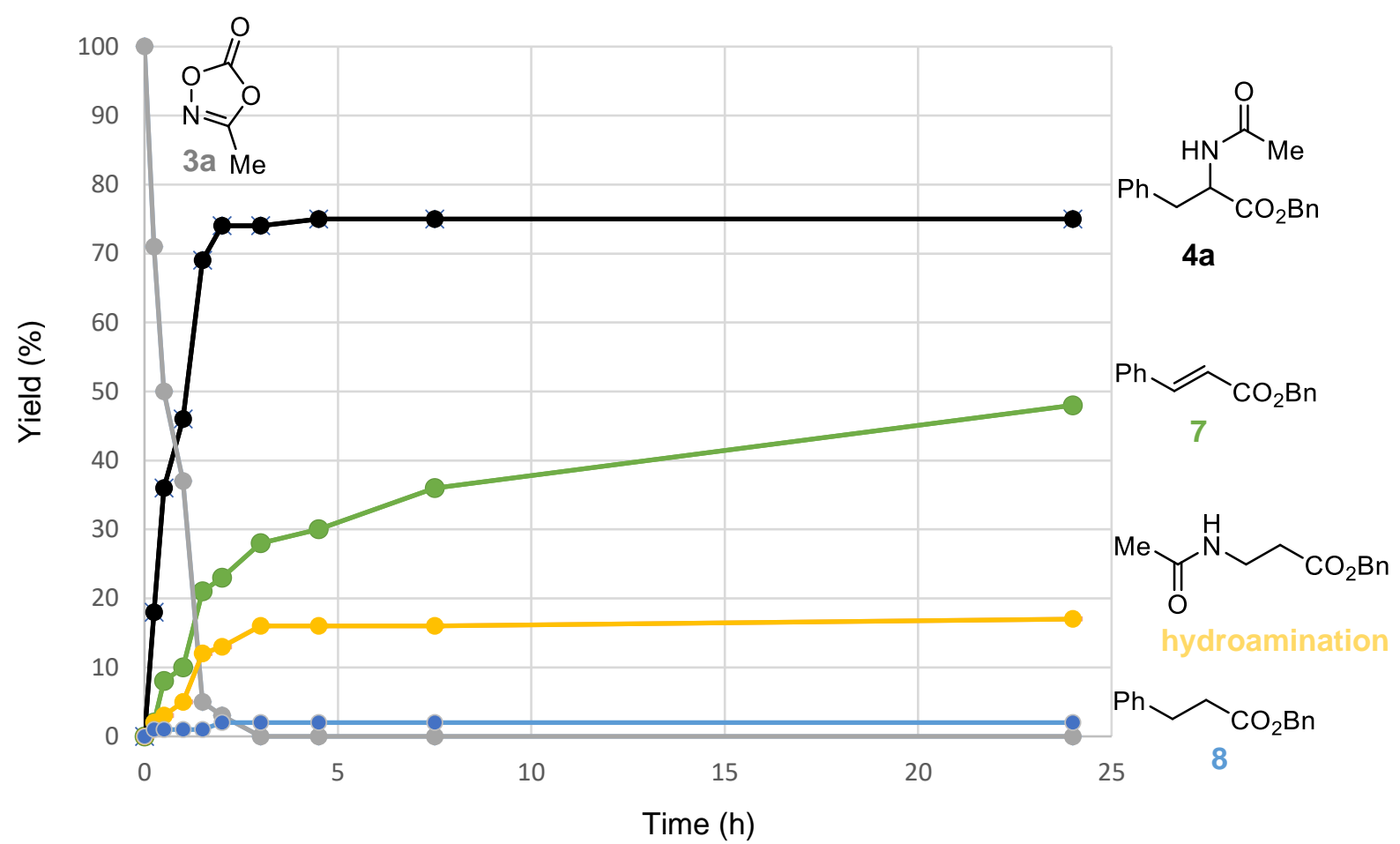

$\left[\mathrm{Cp}^{*} \mathrm{RhCl}_{2}\right]_{2}(0.0050 \mathrm{mmol}, 5 \mathrm{~mol} \%)$ and sodium bicarbonate $(0.03 \mathrm{mmol}, 30 \mathrm{~mol} \%)$, and phenylboronic acid $1 \mathbf{a}(0.25 \mathrm{mmol}, 2.5$ equiv) were measured in a 0.5 -dram vial with a magnetic stir bar. In a separate vial, benzyl acrylate $\mathbf{2 a}(0.3 \mathrm{mmol}, 3$ equiv) and dioxazolone $\mathbf{3 a}(0.1 \mathrm{mmol}, 1$ equiv) were dissolved in $0.10 \mathrm{~mL}$ of $\mathrm{MeOH}$. It was rinsed with an additional $0.07 \mathrm{~mL}$ of $\mathrm{MeOH}$ and transferred again to the first vial. 10 separate reactions were set up and each time the solvent was removed by rotary evaporator and ethyl acetate was added to the residue and filtered through a short pad of celite and washed with ethyl acetate three times. The filtrate was concentrated by rotary evaporator and a crude ${ }^{1} \mathrm{H}$ NMR spectrum was collected with 1,3,5trimethoxybenzene as the internal standard.

\section{Synthesis of benzyl acrylate-3,3- $d_{2}\left(2 \mathrm{a}-d_{2}\right)$}

Benzyl acrylate-3,3- $d_{2} \quad\left(\mathbf{2 a}-\boldsymbol{d}_{2}\right) \quad$ was synthesized following literature ${ }^{11}$ using (benzyloxycarbonylmethyl)triphenylphosphonium bromide and paraformaldehyde- $d_{2}$. To a solution of (benzyloxycarbonylmethyl)triphenylphosphonium bromide $(10 \mathrm{mmol}, 1$ equiv) in a 2:1 mixture of $\mathrm{Et}_{2} \mathrm{O} / \mathrm{H}_{2} \mathrm{O}(40 \mathrm{~mL} / 20 \mathrm{~mL}), \mathrm{K}_{2} \mathrm{CO}_{3}$ was added portionwise over 15 minutes. After stirring 24 hours at $35^{\circ} \mathrm{C}$, the layers were separated, and the water layer was extracted with $\mathrm{Et}_{2} \mathrm{O}(10 \mathrm{~mL})$ three times. The combined 
organic layer was dried with $\mathrm{MgSO}_{4}$ and filtered. To this solution, paraformaldehyde- $d_{2}$ (10.5 mmol, 1.05 equiv) was added and refluxed for 24 hours. After careful removal of the solvent, the crude material was purified by column chromatography using pentane and $\mathrm{Et}_{2} \mathrm{O}$. Yield: $48 \%$, colorless oil. ${ }^{1} \mathbf{H}$ NMR (500 MHz, $\left.\mathrm{CDCl}_{3}\right) \delta 7.44-7.30(\mathrm{~m}, 5 \mathrm{H}), 6.18(\mathrm{bs}, 1 \mathrm{H}), 5.22(\mathrm{~s}, 2 \mathrm{H})$.

\section{Intermolecular competition KIE}

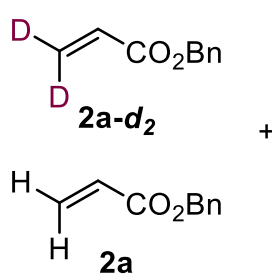

2a

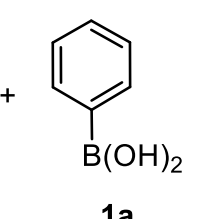

$1 \mathrm{a}$

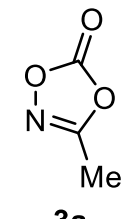

$3 a$
$\left[\mathrm{Cp}^{*} \mathrm{RhCl}_{2}\right]_{2}(5 \mathrm{~mol} \%)$ $\mathrm{NaHCO}_{3}(30 \mathrm{~mol} \%)$

$\mathrm{MeOH}(0.6 \mathrm{M})$,

$30 \min , 25^{\circ} \mathrm{C}$

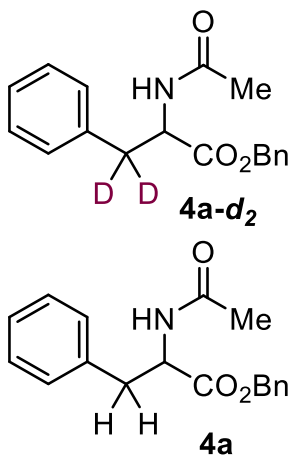

$K_{H} / K_{D}=0.72$, inversed secondary $K I E$

$\left[\mathrm{Cp}^{*} \mathrm{RhCl}_{2}\right]_{2}(0.010 \mathrm{mmol}, 5 \mathrm{~mol} \%)$, sodium bicarbonate $(0.06 \mathrm{mmol}, 30 \mathrm{~mol} \%)$, and phenylboronic acid $1 \mathbf{a}(0.50 \mathrm{mmol}, 2.5$ equiv) were measured in a 1-dram vial with a magnetic stir bar. In a separate vial, $2 \mathbf{a}$ (0.15 mmol, 1.5 equiv) and $\mathbf{2 a}-\boldsymbol{d}_{2}(0.15 \mathrm{mmol}, 1.5$ equiv) were dissolved in $0.20 \mathrm{~mL}$ of $\mathrm{MeOH}$ containing 1,3,5-trimethoxybenzene as standard and transferred to the first vial. It was rinsed with an additional 0.13 $\mathrm{mL}$ of $\mathrm{MeOH}$ and transferred again to the first vial. After the addition of dioxazolone 3a $(0.20 \mathrm{mmol}, 1$ equiv), the reaction mixture was stirred at room temperature for $30 \mathrm{~min} .20 \mathrm{uL}$ aliquot was taken and diluted with $0.5 \mathrm{~mL}$ of MeCN- $d_{3}$ then analyzed by ${ }^{1} \mathrm{H}$ NMR. The KIE value was calculated based on the area of the $4.92 \mathrm{ppm}$ (contribution from both molecules, $\mathbf{4 a}$ and $\mathbf{4 a -}-\boldsymbol{d}_{2}$ ) and at $3.12 \mathrm{ppm}$ (contribution solely from 4a). The experiment was repeated three times and an average KIE value of 0.72 indicates $\mathrm{sp}^{2} \rightarrow \mathrm{sp}^{3}$ hybridization change during the turnover limiting step, which suggests alkene migratory insertion as the turnover limiting step of the reaction. 


\section{Intermolecular parallel KIE}
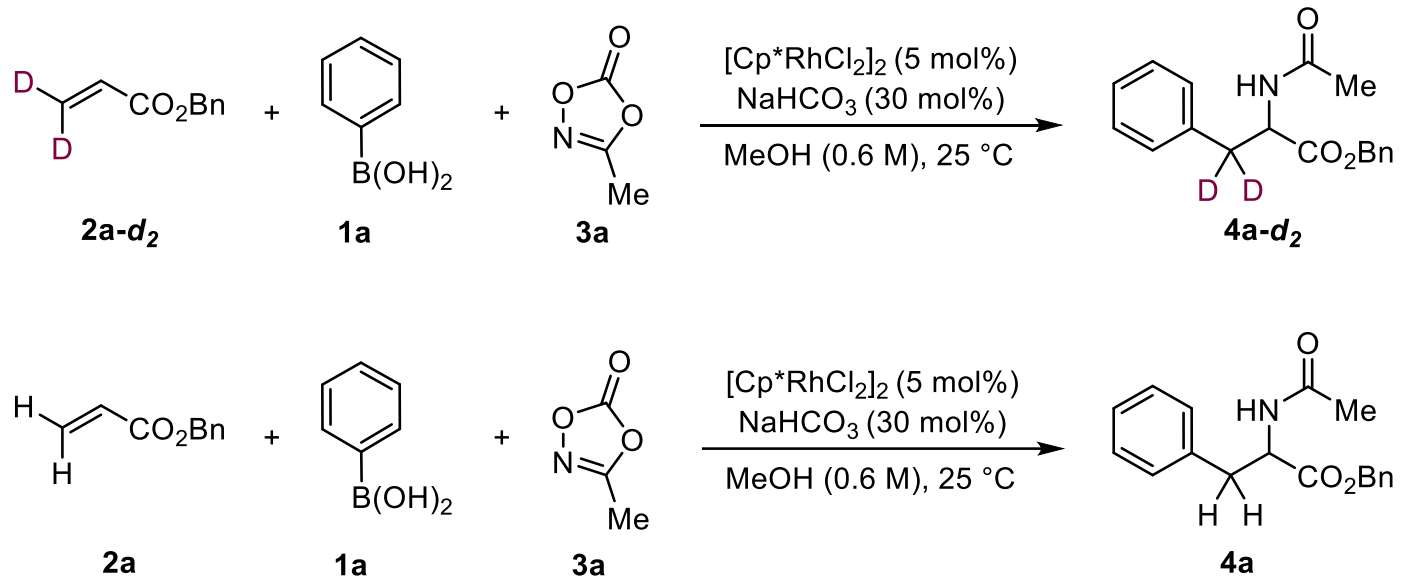

$K_{H} / K_{D}=0.55$, inversed secondary KIE

KIE was measured from two separated reactions, one with benzyl acrylate (2a) containing two C-H bonds and benzyl acrylate-3,3- $d_{2}\left(\mathbf{2 a}-\boldsymbol{d}_{2}\right)$ containing two C-D bonds. [Cp* $\left.\mathrm{RhCl}_{2}\right]_{2}(0.010 \mathrm{mmol}, 5 \mathrm{~mol} \%)$, sodium bicarbonate ( $0.06 \mathrm{mmol}, 30 \mathrm{~mol} \%)$, and phenylboronic acid $1 \mathrm{a}(0.50 \mathrm{mmol}, 2.5$ equiv) were measured in a 1-dram vial with a magnetic stir bar. In a separate vial, 2a (or $\mathbf{2 a - d _ { 2 } )}(0.60 \mathrm{mmol}, 3$ equiv) were dissolved in $0.20 \mathrm{~mL}$ of $\mathrm{MeOH}$ containing 1,3,5-trimethoxybenzene as standard and transferred to the first vial. It was rinsed with an additional $0.13 \mathrm{~mL}$ of $\mathrm{MeOH}$ and transferred again to the first vial. Dioxazolone 3a ( $0.20 \mathrm{mmol}, 1$ equiv) was added to the reaction mixture using a micropipette and the reaction mixture was stirred at room temperature. To monitor the reaction progress, $20 \mathrm{uL}$ aliquot was taken every 5 min and diluted with $0.5 \mathrm{~mL}$ of $\mathrm{MeCN}-d_{3}$. KIE value was calculated by comparing the initial reaction rate (slope) of each reaction (repeated 3 times each) by using ${ }^{1} \mathrm{H}$ NMR. Observed inversed secondary $\mathrm{KIE}\left(\mathrm{K}_{\mathrm{H}} / \mathrm{K}_{\mathrm{D}}\right)$ value of 0.55 suggests $\mathrm{sp}^{2} \rightarrow \mathrm{sp}^{3}$ hybridization change occurs during the turnover limiting step. 


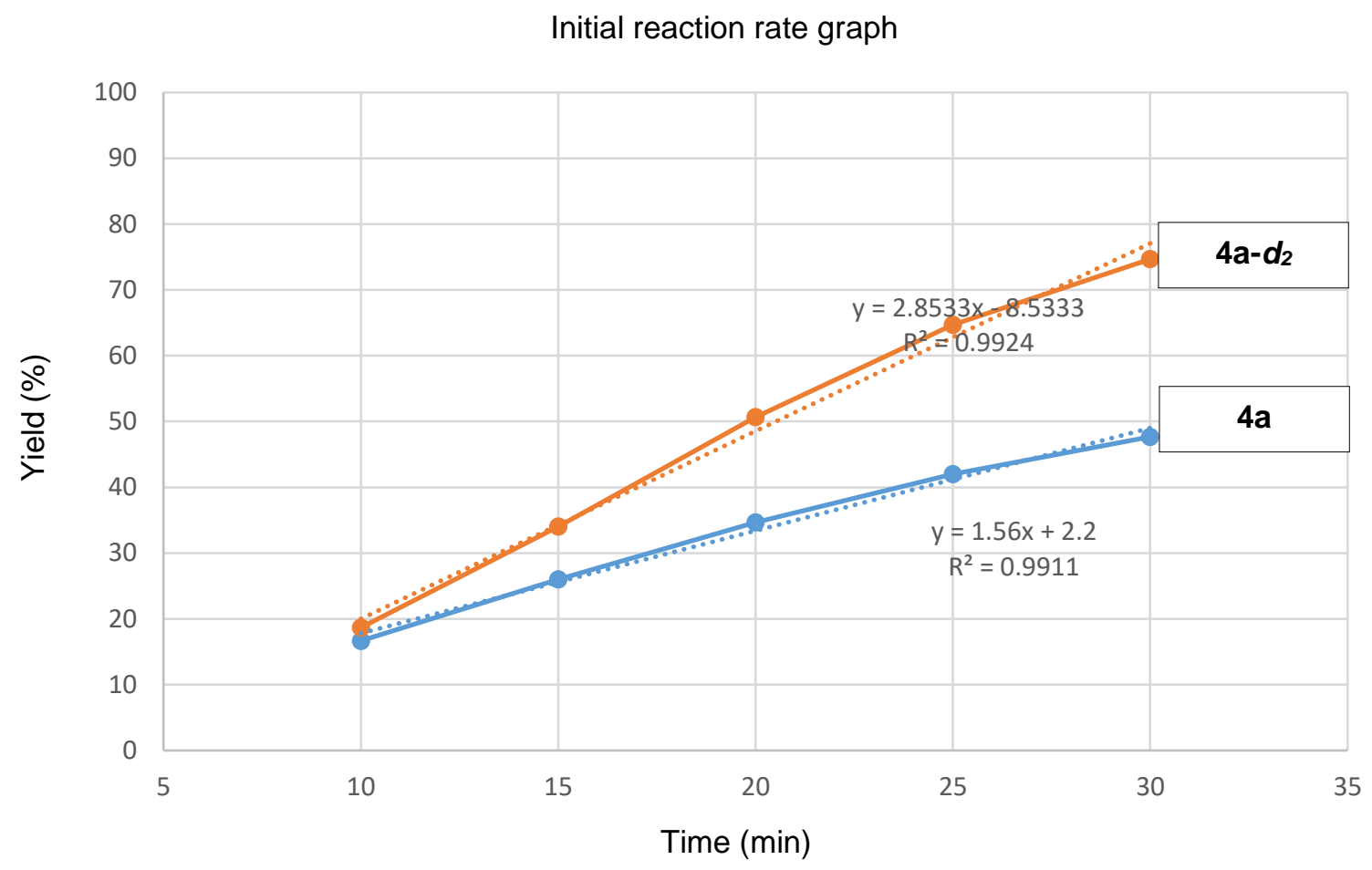

\section{Syn addition of alkenes}

Synthesis of benzyl acrylate-3- $d_{1}\left(2 \mathrm{a}-d_{1}\right)$

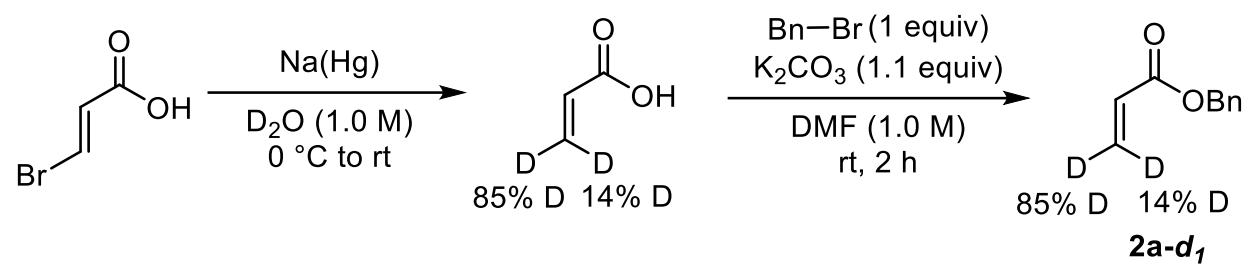

(E)-3-bromoacrylic acid was synthesized following literature. ${ }^{12}$ To a solution of (E)-3-bromoacrylic acid $(1.0 \mathrm{~g}, 6.62 \mathrm{mmol})$ in $\mathrm{D}_{2} \mathrm{O}(1.0 \mathrm{M}), 5.0 \mathrm{~g}$ of $20 \%$ sodium amalgam was added as one portion at $0{ }^{\circ} \mathrm{C}$. After stirring $5 \mathrm{~min}$ at $0{ }^{\circ} \mathrm{C}$ for $5 \mathrm{~min}$, the reaction mixture was warmed to room temperature and stirred additional $30 \mathrm{~min}$. The reaction mixture was decanted and washed with water. The reaction mixture was acidified to $\mathrm{pH} \approx 1$ with $6 \mathrm{M} \mathrm{HCl}$, then extracted with diethyl ether 3 times. The combined organic layer was washed with brine and dried over $\mathrm{MgSO}_{4}$ and solvent was removed by a rotary evaporator. (E)-acrylic-3- $d$ acid was used without further purification (around $20 \%$ of over reduction product was observed). To a mixture of $\mathrm{K}_{2} \mathrm{CO}_{3}$ (1.1 equiv) and benzyl bromide (1 equiv) in DMF (1.0 M), (E)-acrylic-3- $d$ acid was added dropwise at room temperature. After stirring for two hours at room temperature, the reaction mixture was diluted 
with diethyl ether and washed with water and brine. The organic layer was dried with $\mathrm{MgSO}_{4}$ and concentrated. The crude material was purified by column chromatography using pentane and diethyl ether as eluent. 2a-d $\boldsymbol{d}_{\boldsymbol{1}}$. Yield: $20 \%$ (over two steps). Colorless oil. ${ }^{1} \mathbf{H}$ NMR (400 MHz, $\left.\mathrm{CDCl}_{3}\right) \delta 7.44-7.30$ (m, $5 \mathrm{H}), 6.44(\mathrm{~d}, J=17.3 \mathrm{~Hz}, 0.86 \mathrm{H}), 6.17(\mathrm{dt}, J=17.2,1.5 \mathrm{~Hz}, 1 \mathrm{H}), 5.84(\mathrm{~d}, J=10.5 \mathrm{~Hz}, 0.15 \mathrm{H})$.

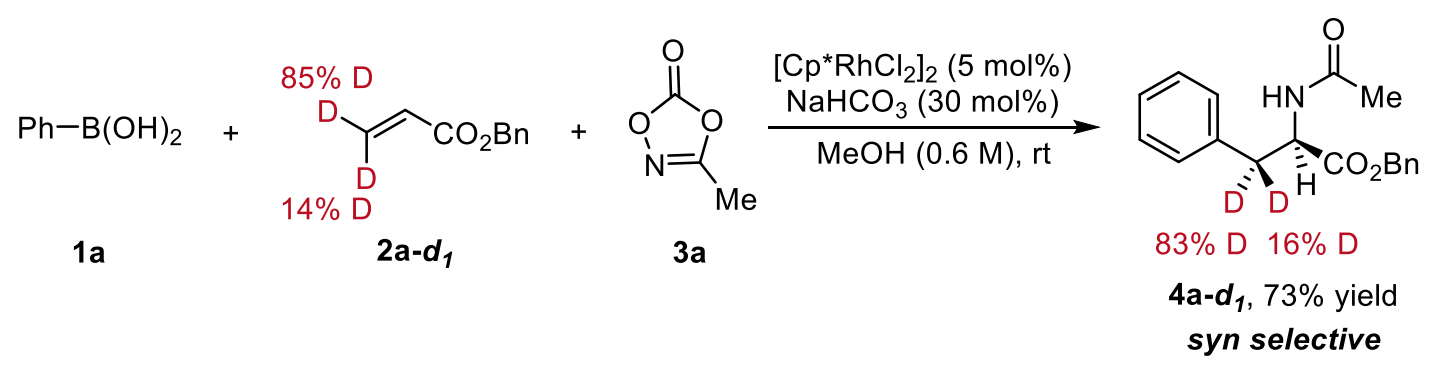

To a solution of $\left[\mathrm{Cp}^{*} \mathrm{RhCl}_{2}\right]_{2}(0.05 \mathrm{mmol}, 5 \mathrm{~mol} \%)$, sodium bicarbonate $(0.30 \mathrm{mmol}, 30 \mathrm{~mol} \%)$, and phenylboronic acid $\mathbf{1 a}\left(2.5 \mathrm{mmol}, 2.5\right.$ equiv) in $\mathrm{MeOH}(1.7 \mathrm{~mL}, 0.6 \mathrm{M}), \mathbf{2 a}-\boldsymbol{d}_{\boldsymbol{l}}(3.0 \mathrm{mmol}, 3.0$ equiv) and 3a $(1.0 \mathrm{mmol}, 1$ equiv) were added at room temperature and stirred overnight. The volatiles were removed by rotary evaporator and ethyl acetate was added and filtered through short pad of celite. After removing solvent using rotary evaporator, the crude material was purified by column chromatography using ethyl acetate and hexane as eluent to obtain $4 \mathbf{4 a -} \boldsymbol{d}_{\boldsymbol{l}}$. Yield: $73 \%$, white solid. ${ }^{1} \mathbf{H}$ NMR (500 MHz, $\left.\mathrm{CD}_{3} \mathrm{CN}\right) \delta 7.40$ $-7.22(\mathrm{~m}, 8 \mathrm{H}), 7.18-7.14(\mathrm{~m}, 2 \mathrm{H}), 6.67(\mathrm{~s}, 1 \mathrm{H}), 5.12-5.06(\mathrm{~m}, 2 \mathrm{H}), 4.63(\mathrm{t}, J=7.8 \mathrm{~Hz}, 1 \mathrm{H}), 3.07$ (d, $J$ $=6.1 \mathrm{~Hz}, 0.17 \mathrm{H}), 2.94(\mathrm{~d}, J=8.0 \mathrm{~Hz}, 0.84 \mathrm{H}), 1.84(\mathrm{~s}, 3 \mathrm{H})$.

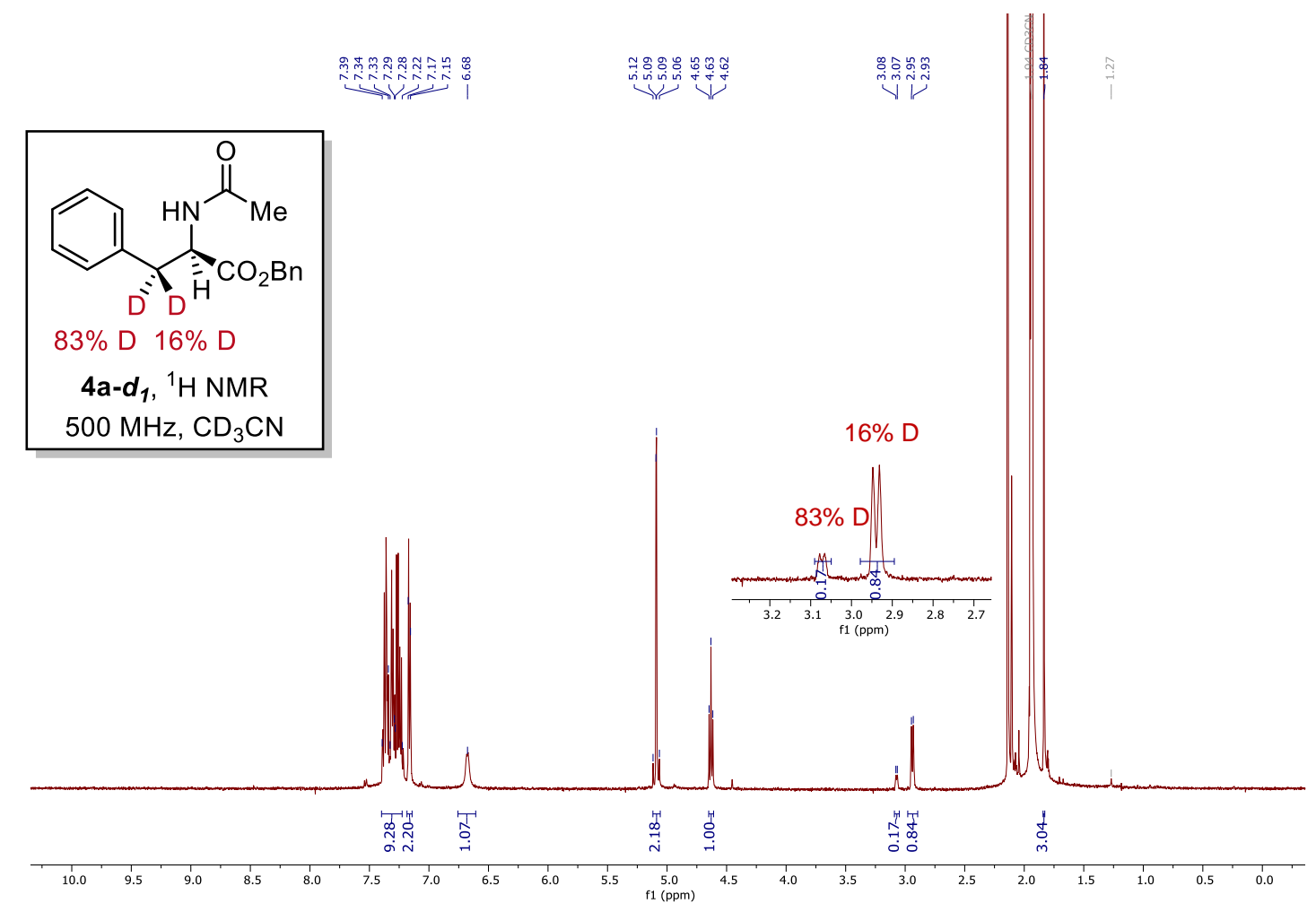




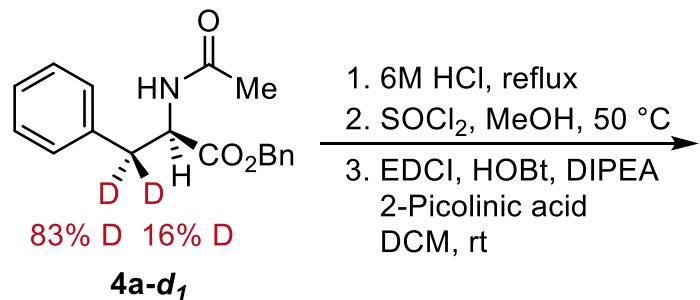

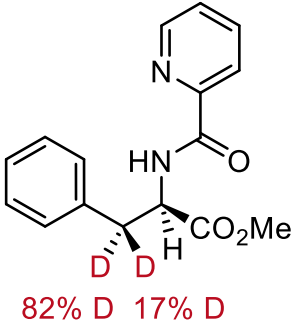

$4 a^{\prime}-d_{1}$

$69 \%$ yield

(over 3 steps)
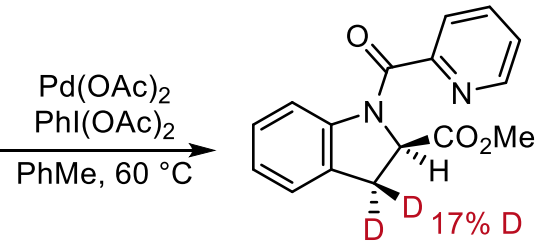

$82 \%$ D

$11-d_{1}, 81 \%$ yield

$6 \mathrm{M} \mathrm{HCl}(0.1 \mathrm{M})$ was added to $4 \mathbf{a}-\boldsymbol{d}_{\boldsymbol{I}}(1.38 \mathrm{mmol})$ and refluxed for 4 hours. After removing volatiles, diethyl ether was added, and the white solid was collected by filteration. The resulting solid was washed with diethyl ether and dried under high vacuum. The deprotected product was dissolved in $\mathrm{MeOH}(0.25 \mathrm{M})$ and $\mathrm{SOCl}_{2}$ (1.5 equiv) was added dropwise at $0{ }^{\circ} \mathrm{C}$. The reaction mixture was warmed to room temperature and heated at $50^{\circ} \mathrm{C}$ for 3 hours. The volatiles were removed by a rotary evaporator and diethyl ether was added. After stirring for $10 \mathrm{~min}$ at $0{ }^{\circ} \mathrm{C}$, the white solid was filtered and washed with diethyl ether to give crude deuterated phenylalanine methyl ester hydrochloride. The crude material (1 equiv) and 2-picolinic acid (1.1 equiv) were dissolved in DCM (0.2 M) and HOBt hydrate (1.1 equiv) and DIPEA (3.0 equiv) were added at room temperature. The reaction mixture was cooled to $0{ }^{\circ} \mathrm{C}$ and EDCI (1.1 equiv) was added. After stirring for $5 \mathrm{~min}$, the reaction mixture was warmed to room temperature and stirred overnight. Water was added and extracted with DCM three times. The combined organic layer was washed with water and brine and dried with $\mathrm{Na}_{2} \mathrm{SO}_{4}$. After removing the solvent, the crude material was purified by column chromatography using ethyl acetate and hexane as eluent to obtain $\mathbf{4 a} \mathbf{a}^{\prime}-\boldsymbol{d}_{\boldsymbol{l}}$. Yield: 69\% (over three steps). White solid. ${ }^{1} \mathbf{H}$ NMR $\left(400 \mathrm{MHz}, \mathrm{CDCl}_{3}\right) \delta 8.55$ (ddd, $\left.J=4.8,1.8,0.9 \mathrm{~Hz}, 1 \mathrm{H}\right), 8.47(\mathrm{~d}, J=8.4 \mathrm{~Hz}, 1 \mathrm{H})$, $8.16(\mathrm{dt}, J=7.8,1.1 \mathrm{~Hz}, 1 \mathrm{H}), 7.83(\mathrm{td}, J=7.7,1.7 \mathrm{~Hz}, 1 \mathrm{H}), 7.42(\mathrm{ddd}, J=7.6,4.8,1.3 \mathrm{~Hz}, 1 \mathrm{H}), 7.33-$ $7.14(\mathrm{~m}, 5 \mathrm{H}), 5.06(\mathrm{dd}, J=8.4,6.3 \mathrm{~Hz}, 1 \mathrm{H}), 3.73(\mathrm{~s}, 3 \mathrm{H}), 3.24(\mathrm{~d}, J=5.8 \mathrm{~Hz}, 0.18 \mathrm{H}), 3.21(\mathrm{~d}, J=6.5 \mathrm{~Hz}$, $0.83 \mathrm{H})$. 


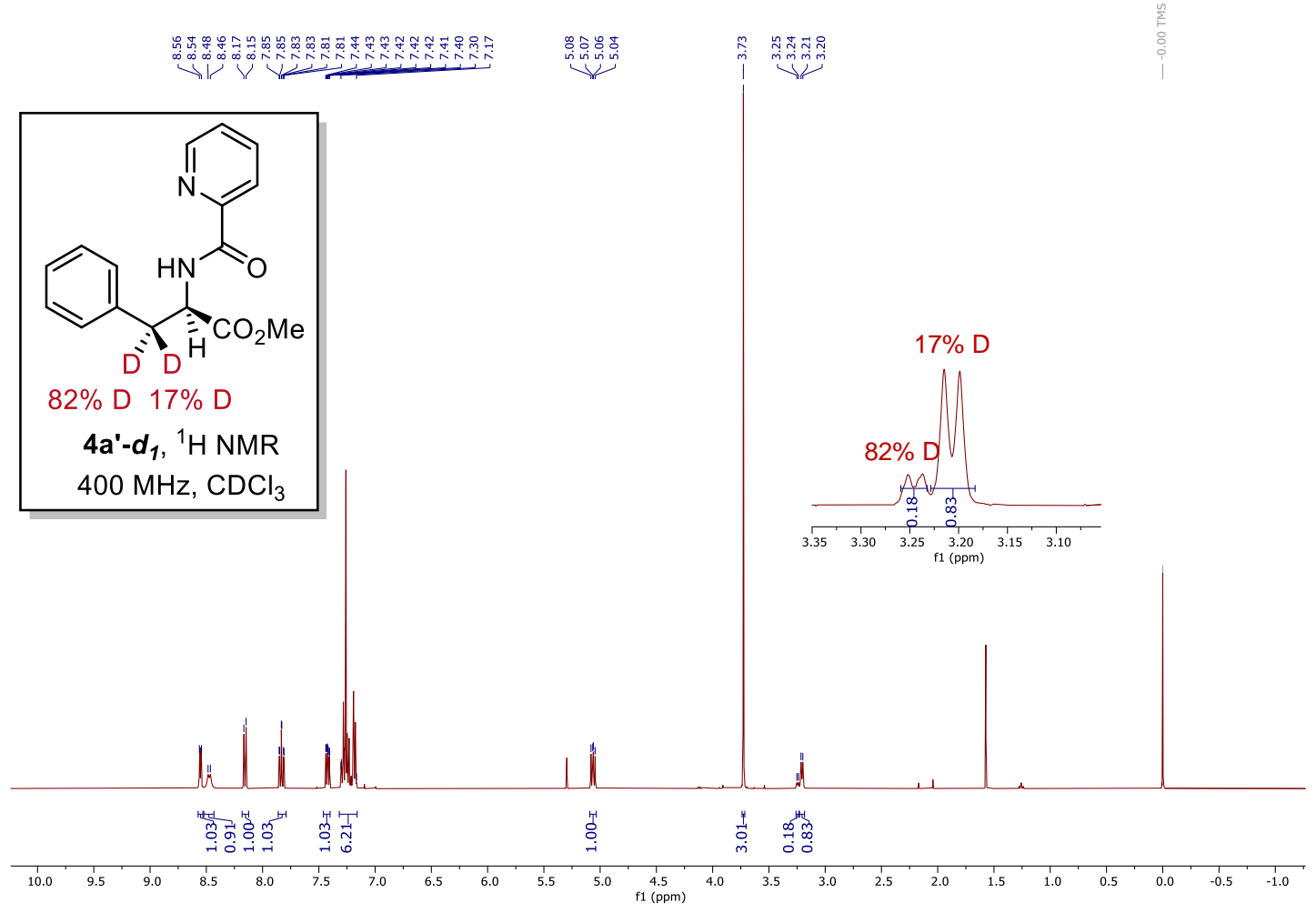

9a- $\boldsymbol{d}_{\boldsymbol{I}}$ was prepared following the literature. ${ }^{13} \mathrm{In}$ a $20 \mathrm{~mL}$ vial, $\mathrm{Pd}(\mathrm{OAc})_{2}(2 \mathrm{~mol} \%, 0.018 \mathrm{mmol})$ and $\mathrm{PhI}(\mathrm{OAc})_{2}$ (2 equiv. $\left.1.78 \mathrm{mmol}\right)$ were measured in the glovebox. In a separate flask, $4 \mathbf{a}^{\prime}-\boldsymbol{d}_{\boldsymbol{l}}$ (1 equiv, 0.89 $\mathrm{mmol})$ was dissolved in toluene $(0.125 \mathrm{M})$ and transferred to the first vial. After purging with Ar for 10 min, the vial was sealed and heated at $60{ }^{\circ} \mathrm{C}$ for 24 hours. The reaction mixture was concentrated and purified by column chromatography using ethyl acetate and hexane as eluent. 11a- $\boldsymbol{d}_{\boldsymbol{l}}$. Yield: $81 \%$, white solid. ${ }^{1} \mathbf{H}$ NMR $\left(500 \mathrm{MHz}\right.$, toluene- $\left.d_{8}\right) \delta 8.85(\mathrm{~d}, J=8.2 \mathrm{~Hz}, 1 \mathrm{H}), 8.14(\mathrm{~d}, J=4.8 \mathrm{~Hz}, 1 \mathrm{H}), 8.09(\mathrm{~d}, J=7.9$ $\mathrm{Hz}, 1 \mathrm{H}), 7.15-7.16(\mathrm{~m}, 2 \mathrm{H}), 6.89-6.81(\mathrm{~m}, 2 \mathrm{H}), 7.04-6.96(\mathrm{~m}, 2 \mathrm{H}), 6.64$ (ddd, $J=7.6,4.7,1.2 \mathrm{~Hz}$, 1H), $5.77-5.71(\mathrm{~m}, 1 \mathrm{H}), 3.18(\mathrm{~s}, 3 \mathrm{H}), 3.04(\mathrm{~d}, J=11.8 \mathrm{~Hz}, 0.18 \mathrm{H}), 2.93(\mathrm{~s}, 0.83 \mathrm{H})$. 


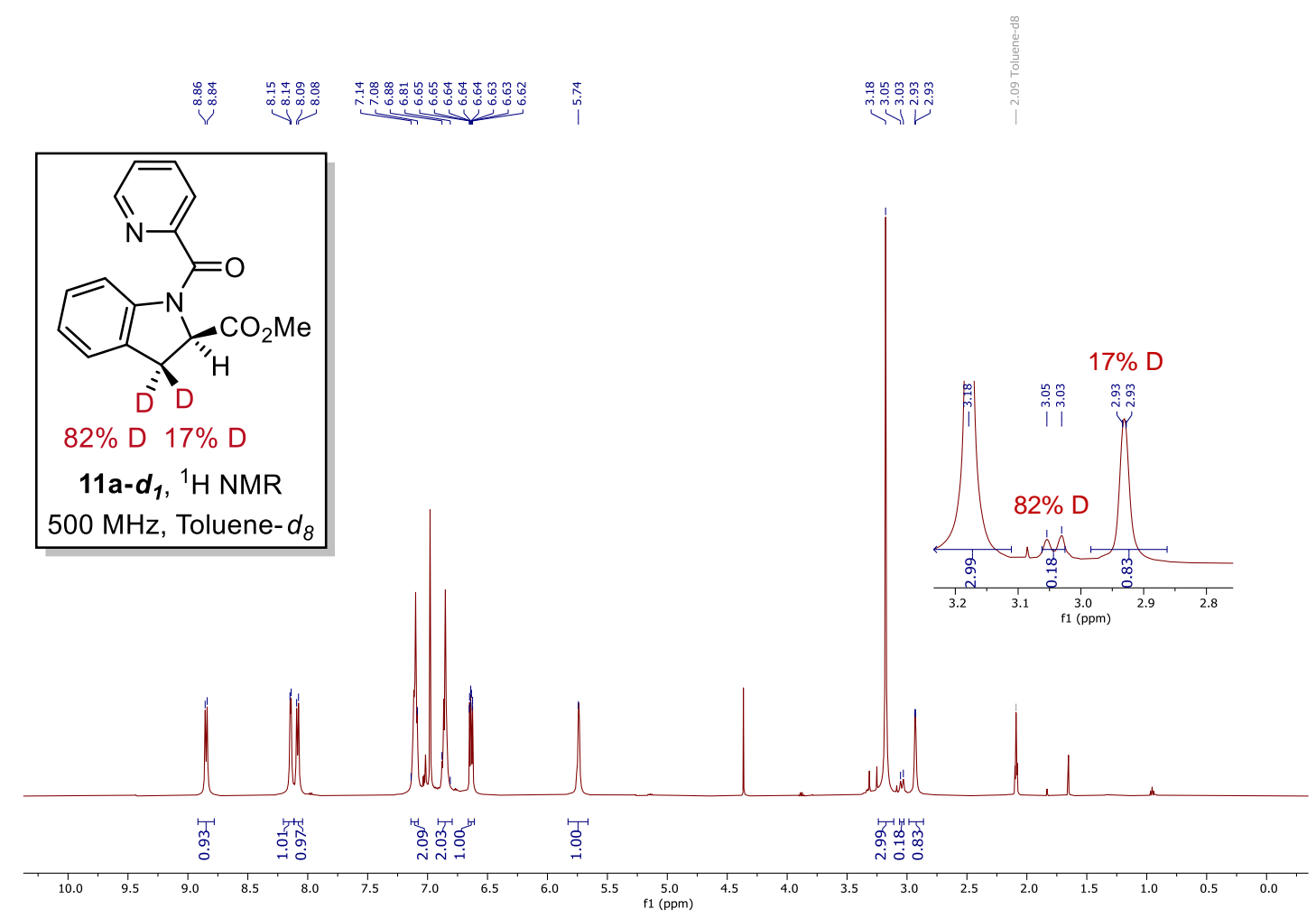

11a was synthesized from phenylalanine using same procedure described above. 11a. White solid. ${ }^{1} \mathbf{H}$ NMR $\left(500 \mathrm{MHz}\right.$, toluene- $\left.d_{8}\right) \delta 8.86(\mathrm{~d}, J=8.2 \mathrm{~Hz}, 1 \mathrm{H}), 8.14(\mathrm{~d}, J=4.5 \mathrm{~Hz}, 1 \mathrm{H}), 8.09(\mathrm{~d}, J=7.9 \mathrm{~Hz}, 1 \mathrm{H})$, $7.09(\mathrm{td}, J=9.6,7.8,6.0 \mathrm{~Hz}, 2 \mathrm{H}), 6.89-6.81(\mathrm{~m}, 2 \mathrm{H}), 6.62(\mathrm{dd}, J=7.7,4.8 \mathrm{~Hz}, 1 \mathrm{H}), 5.75(\mathrm{dd}, J=10.9$, $3.2 \mathrm{~Hz}, 1 \mathrm{H}), 3.17$ (s, 3H), 3.05 (dd, $J=16.4,10.9 \mathrm{~Hz}, 1 \mathrm{H}), 2.95$ (dd, $J=16.3,3.2 \mathrm{~Hz}, 1 \mathrm{H})$. The relative stereochemistry was assigned based on the literature. ${ }^{14}$ Benzylic proton with $10.9 \mathrm{~Hz}$ coupling constant was assigned as cis proton and proton with $3.2 \mathrm{~Hz}$ was assigned as trans proton which confirms the syncarboamination of the reaction.
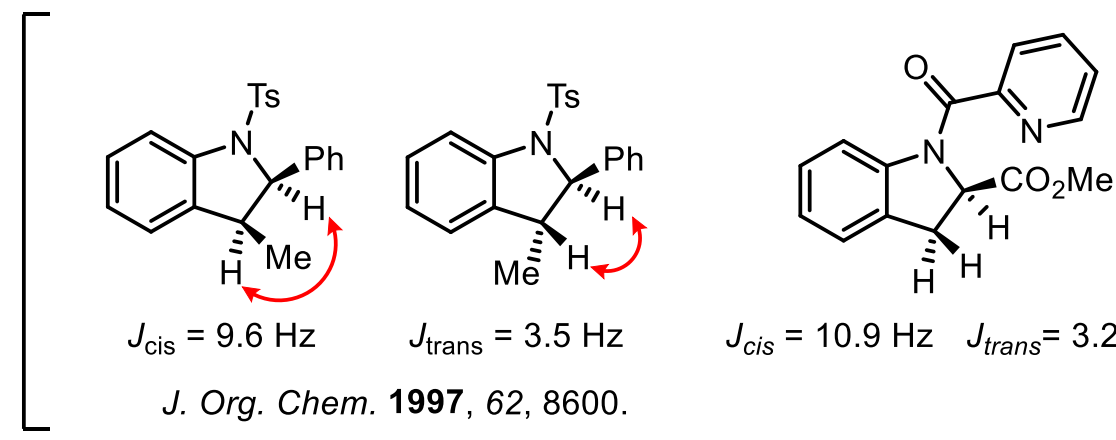

$$
\begin{gathered}
J_{\text {cis }}=9.6 \mathrm{~Hz} \quad J_{\text {trans }}=3.5 \mathrm{~Hz} \\
\text { J. Org. Chem. 1997, 62, } 8600 .
\end{gathered}
$$$$
J_{\text {cis }}=10.9 \mathrm{~Hz} \quad J_{\text {trans }}=3.2 \mathrm{~Hz}
$$ 


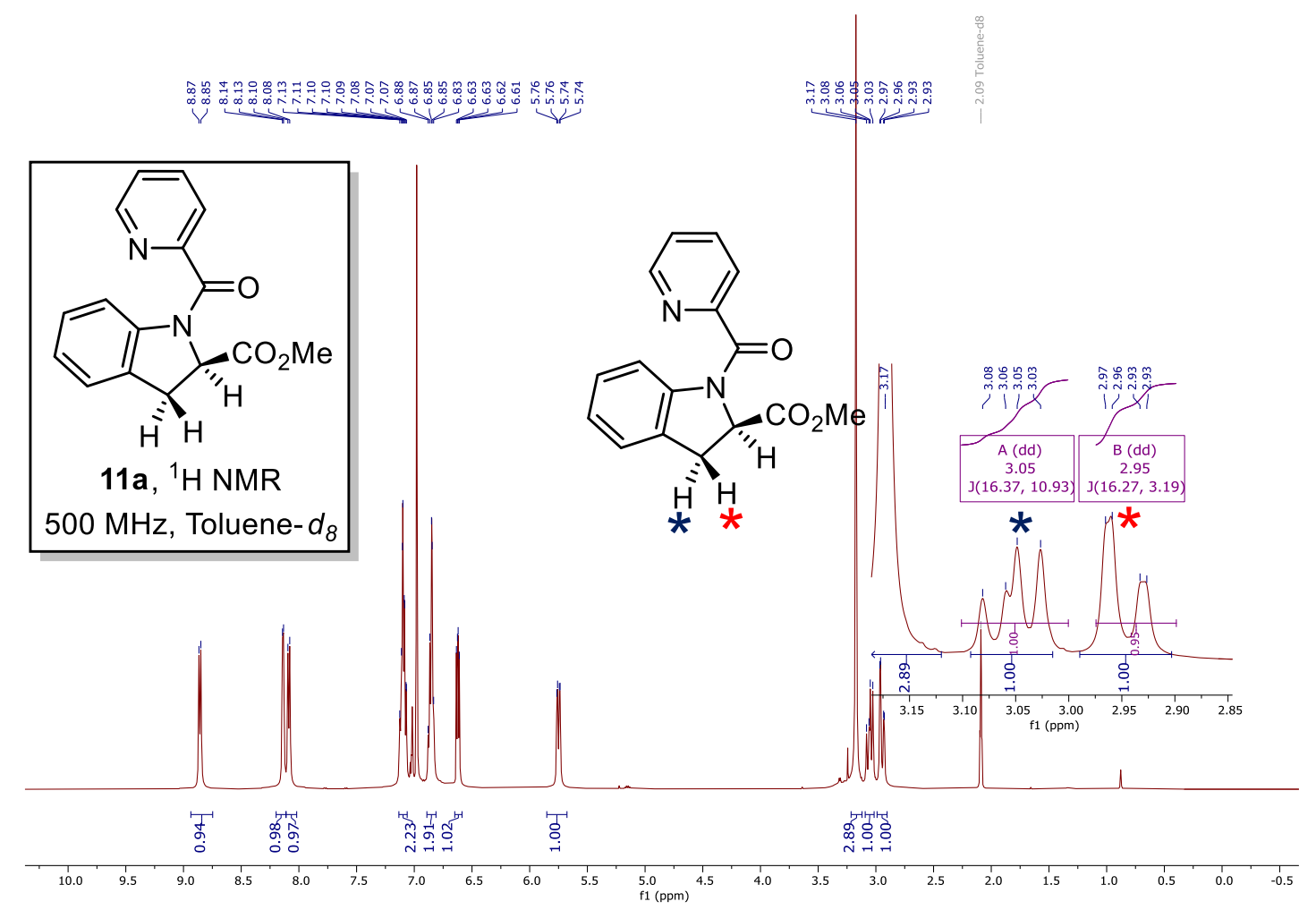




\section{Limitation}

<Unsuccessful alkene coupling partners>

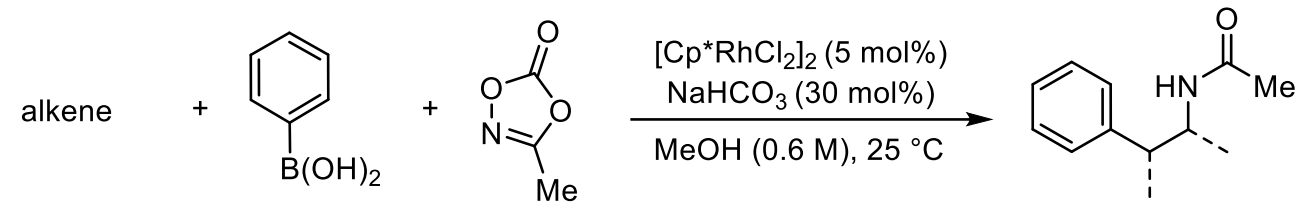

$1 \mathrm{a} \quad 3 a$

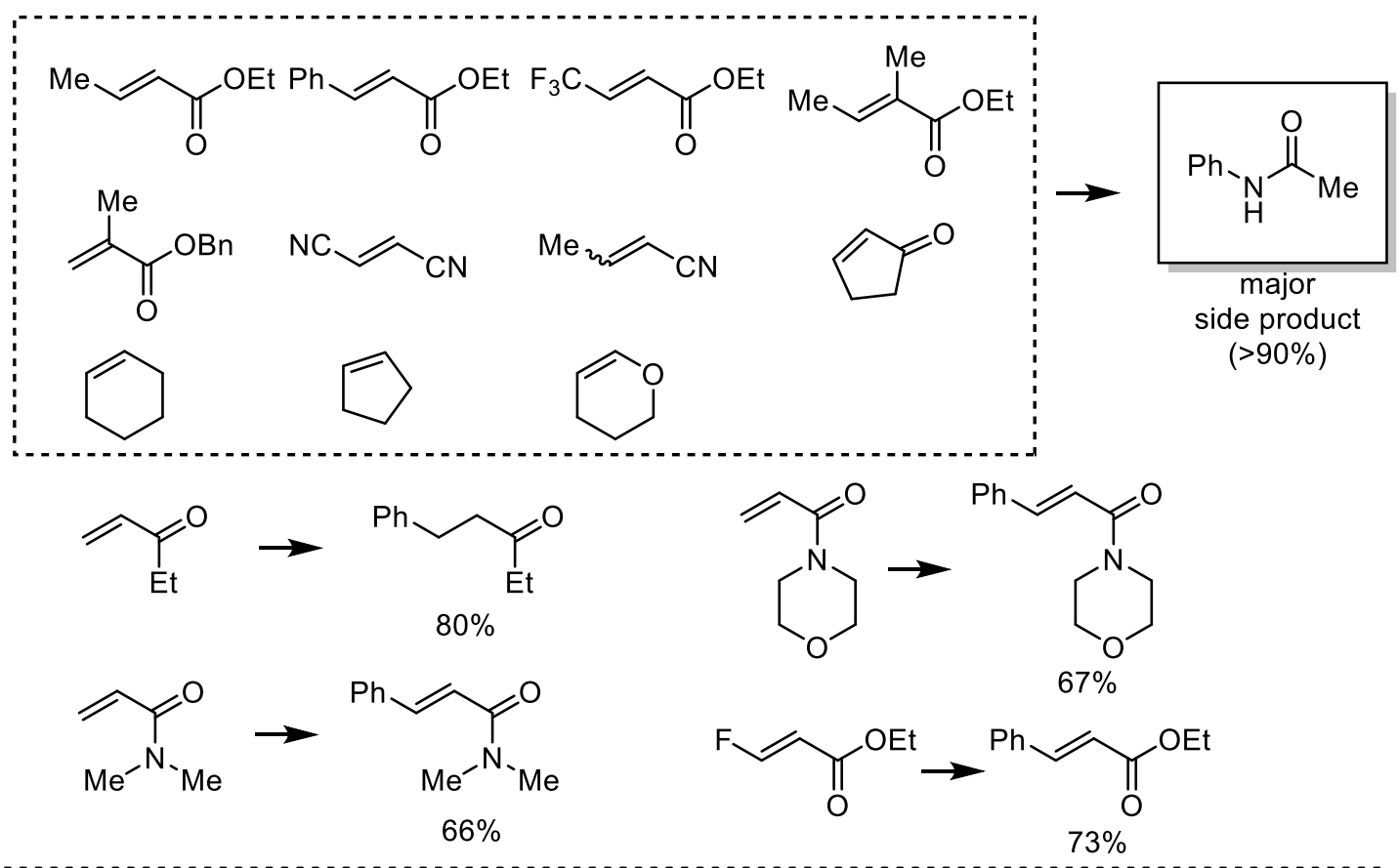

<Unsuccessful boronic acids and dioxazolones><smiles>OB(O)C1CCCCC1</smiles><smiles>CCC/C=C/B(O)O</smiles><smiles>Cc1cccc(C)c1B(O)O</smiles><smiles>OB(O)c1c(F)c(F)c(F)c(F)c1F</smiles><smiles>OB(O)c1ccco1</smiles><smiles>OB(O)c1ccoc1</smiles><smiles>OB(O)c1ccccn1</smiles><smiles>OB(O)c1ccc2cccnc2c1</smiles><smiles>O=c1onc(-c2ccccc2)o1</smiles><smiles>CC(C)(C)c1noc(=O)o1</smiles><smiles>O=c1onc(Br)o1</smiles> 


\section{Reference}

(1) Chanthamath, S.; Takaki, S.; Shibatomi, K.; Iwasa, S. Highly Stereoselective Cyclopropanation of $\alpha, \beta$-Unsaturated Carbonyl Compounds with Methyl (Diazoacetoxy)Acetate Catalyzed by a Chiral Ruthenium(II) Complex. Angew. Chemie Int. Ed. 2013, 52, 5818-5821.

(2) Yang, W.; Luo, R.; Yang, D. Iridium-Catalyzed Asymmetric Ring-Opening of Oxabenzonorbornadienes with N-Substituted Piperazine Nucleophiles. Molecules 2015, 20, 21103.

(3) Lautens, M.; Fagnou, K.; Zunic, V. An Expedient Enantioselective Route to Diaminotetralins: Application in the Preparation of Analgesic Compounds. Org. Lett. 2002, 4, 3465-3468.

(4) Phan, D. H. T.; Kou, K. G. M.; Dong, V. M. Enantioselective Desymmetrization of Cyclopropenes by Hydroacylation. J. Am. Chem. Soc. 2010, 132, 16354-16355.

(5) Cheng, X.; Zhu, L.; Lin, M.; Chen, J.; Huang, X. Rapid Access to Cyclopentadiene Derivatives through Gold-Catalyzed Cycloisomerization of Ynamides with Cyclopropenes by Preferential Activation of Alkenes over Alkynes. Chem. Commun. 2017, 53, 3745-3748.

(6) Lei, H.; Rovis, T. Ir-Catalyzed Intermolecular Branch-Selective Allylic C-H Amidation of Unactivated Terminal Olefins. J. Am. Chem. Soc. 2019, 141, 2268-2273.

(7) Hong, S. Y.; Park, Y.; Hwang, Y.; Kim, Y. B.; Baik, M.-H.; Chang, S. Selective Formation of $\gamma$ Lactams via C-H Amidation Enabled by Tailored Iridium Catalysts. Science (80-. ). 2018, 359, 1016 LP - 1021.

(8) Farr, C. M. B.; Kazerouni, A. M.; Park, B.; Poff, C. D.; Won, J.; Sharp, K. R.; Baik, M.-H.; Blakey, S. B. Designing a Planar Chiral Rhodium Indenyl Catalyst for Regio- and Enantioselective Allylic C-H Amidation. J. Am. Chem. Soc. 2020, 142, 13996-14004.

(9) Lutjen, A. B.; Quirk, M. A.; Barbera, A. M.; Kolonko, E. M. Synthesis of (E)-Cinnamyl Ester Derivatives via a Greener Steglich Esterification. Bioorg. Med. Chem. 2018, 26, 5291-5298.

(10) Chen, H.; Xu, X.; Liu, L.; Tang, G.; Zhao, Y. Phosphorus Oxychloride as an Efficient Coupling Reagent for the Synthesis of Esters, Amides and Peptides under Mild Conditions. RSC Adv. 2013, $3,16247-16250$.

(11) Wenzel, S. C.; Williamson, R. M.; Grünanger, C.; Xu, J.; Gerth, K.; Martinez, R. A.; Moss, S. J.; Carroll, B. J.; Grond, S.; Unkefer, C. J.; et al. On the Biosynthetic Origin of MethoxymalonylAcyl Carrier Protein, the Substrate for Incorporation of "Glycolate" Units into Ansamitocin and Soraphen A. J. Am. Chem. Soc. 2006, 128, 14325-14336. 
(12) Ouellet, R. ,3'a-Dihydroxycyclopent-1'. 1976, No. 10, 2-11.

(13) He, G.; Lu, C.; Zhao, Y.; Nack, W. A.; Chen, G. Improved Protocol for Indoline Synthesis via Palladium-Catalyzed Intramolecular C(Sp2)-H Amination. Org. Lett. 2012, 14, 2944-2947.

(14) McComas, C. C.; Gilbert, E. J.; Van Vranken, D. L. Stereochemistry of 3-Alkylindole

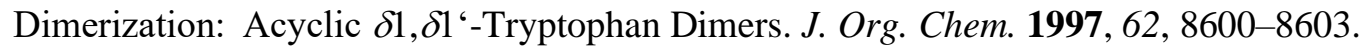




\section{NMR Spectra}
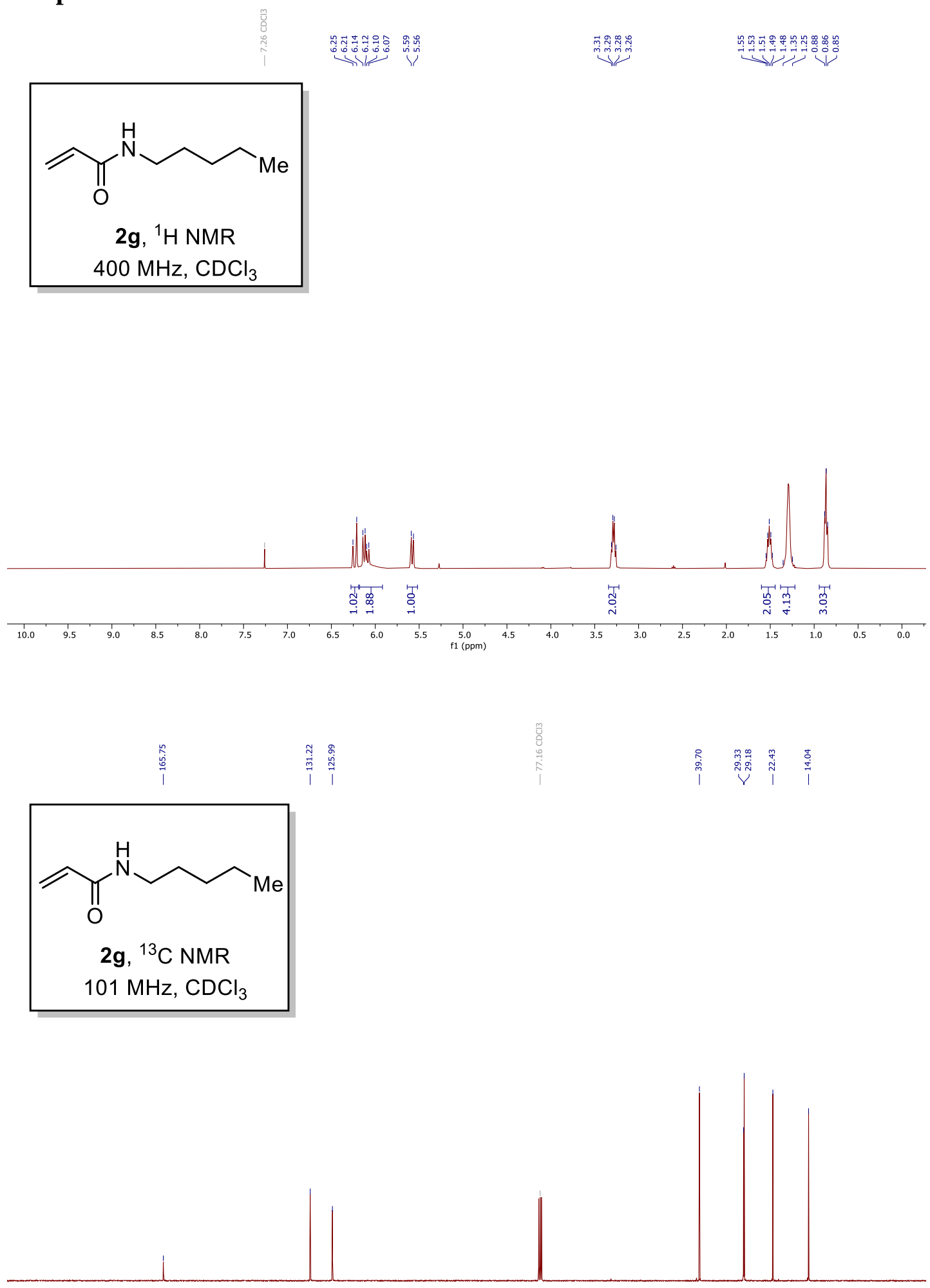

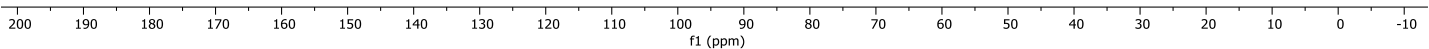



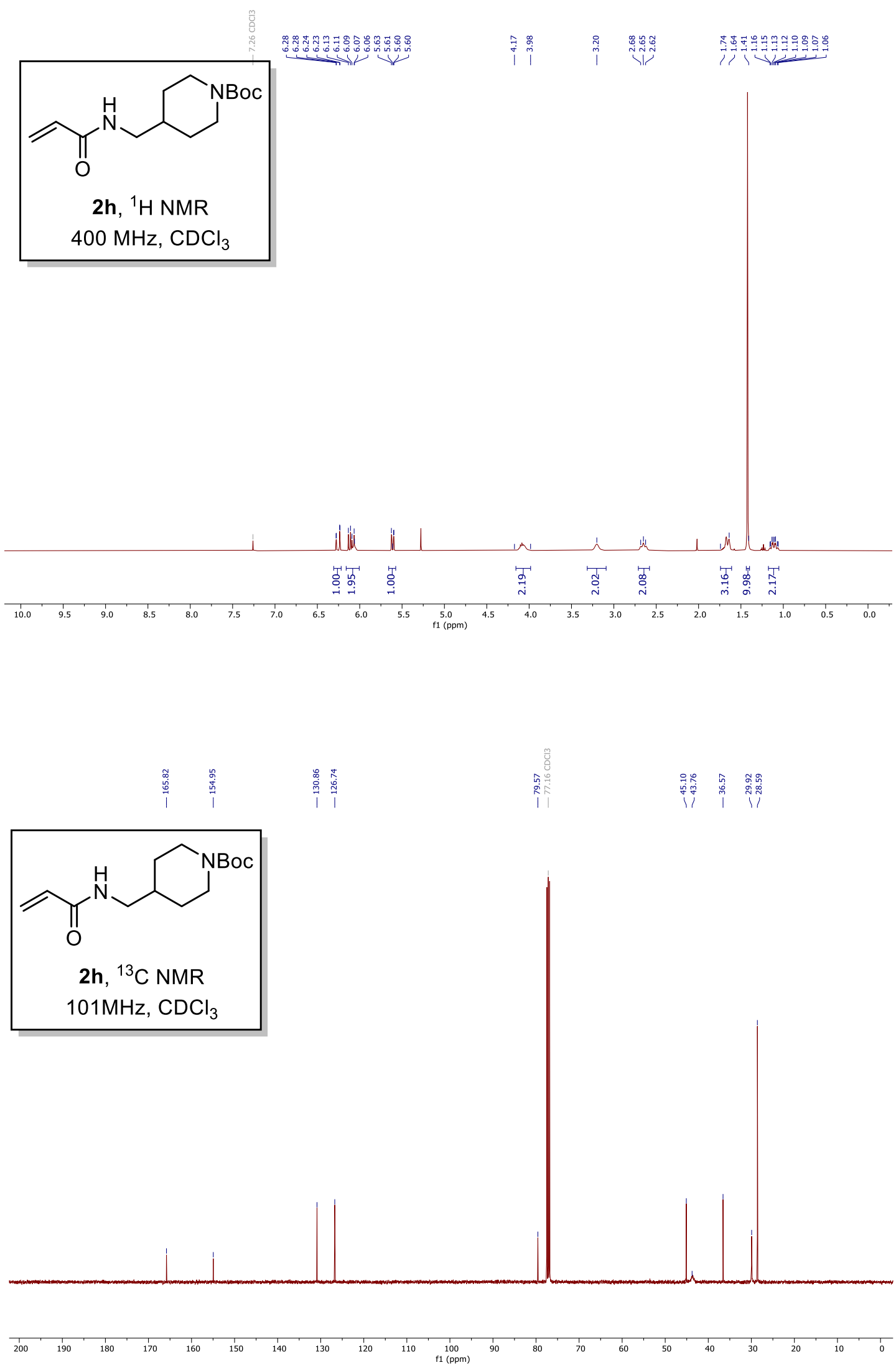

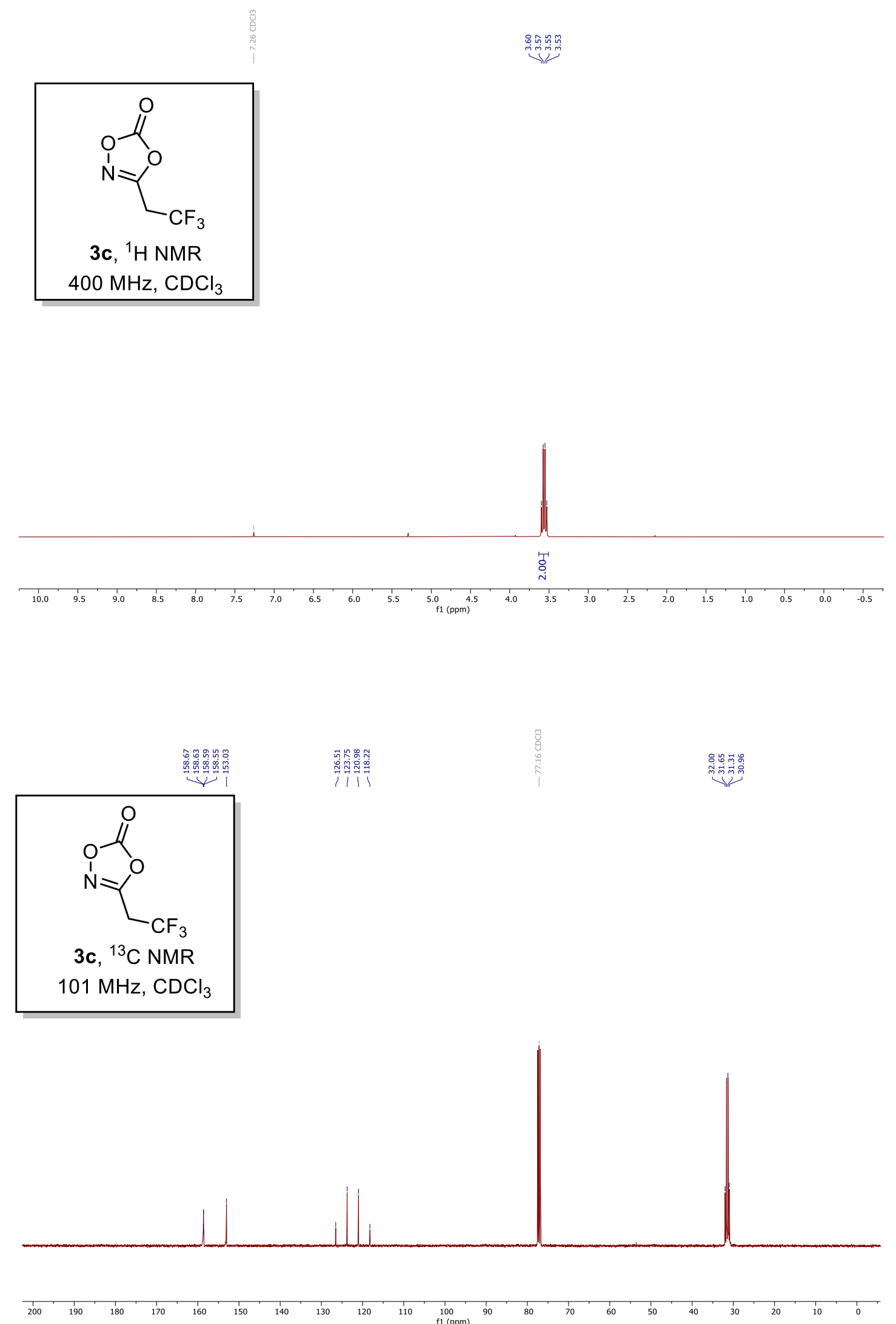


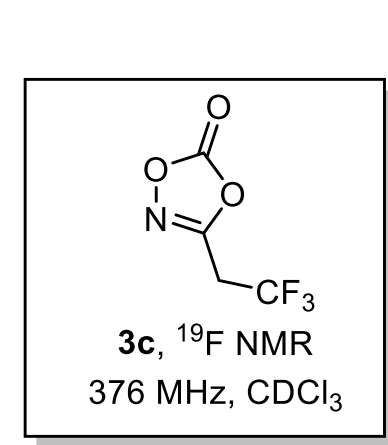

3c, ${ }^{19} \mathrm{~F}$ NMR

$376 \mathrm{MHz}, \mathrm{CDCl}_{3}$

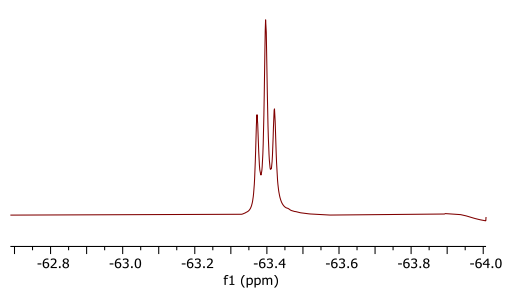



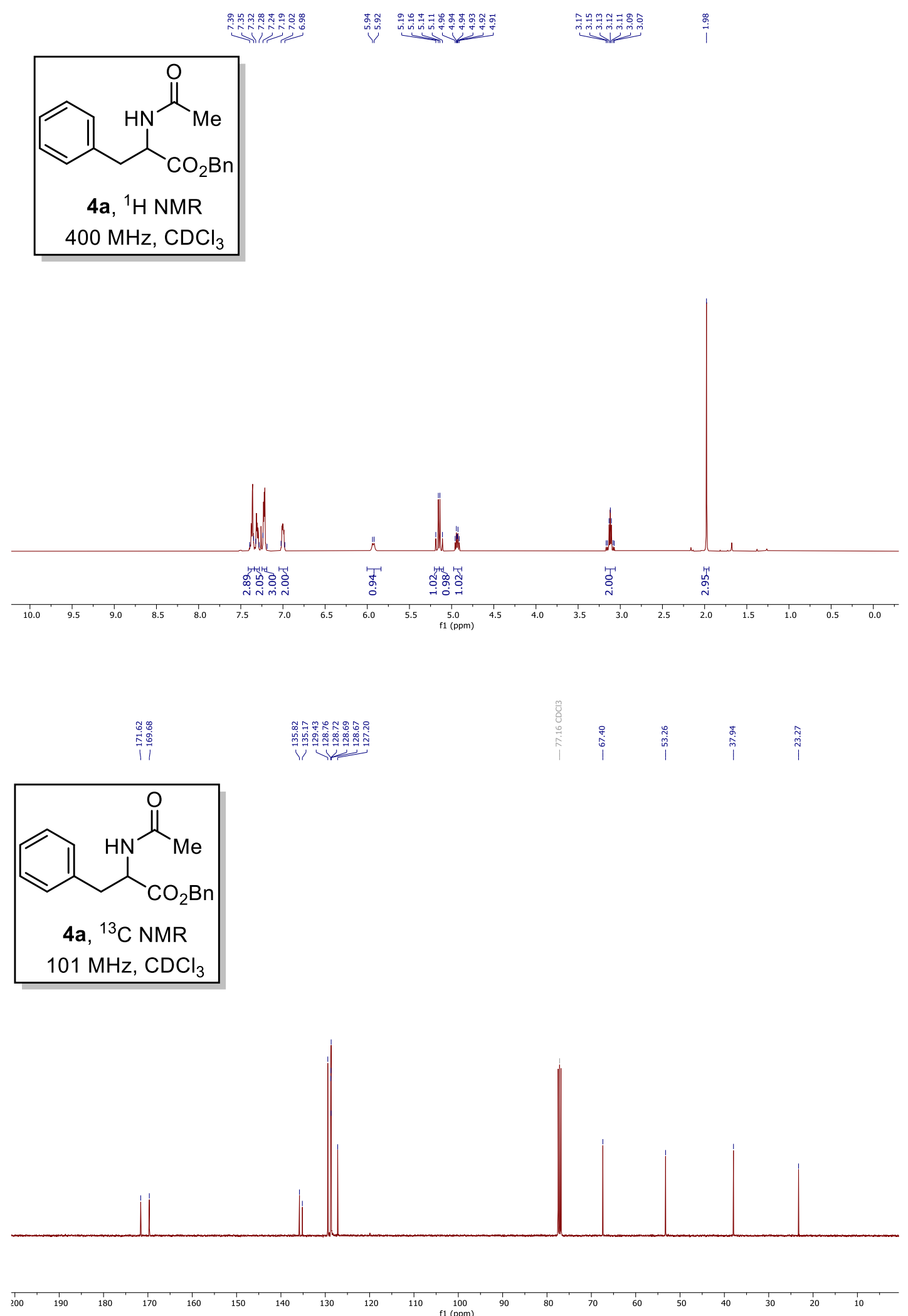

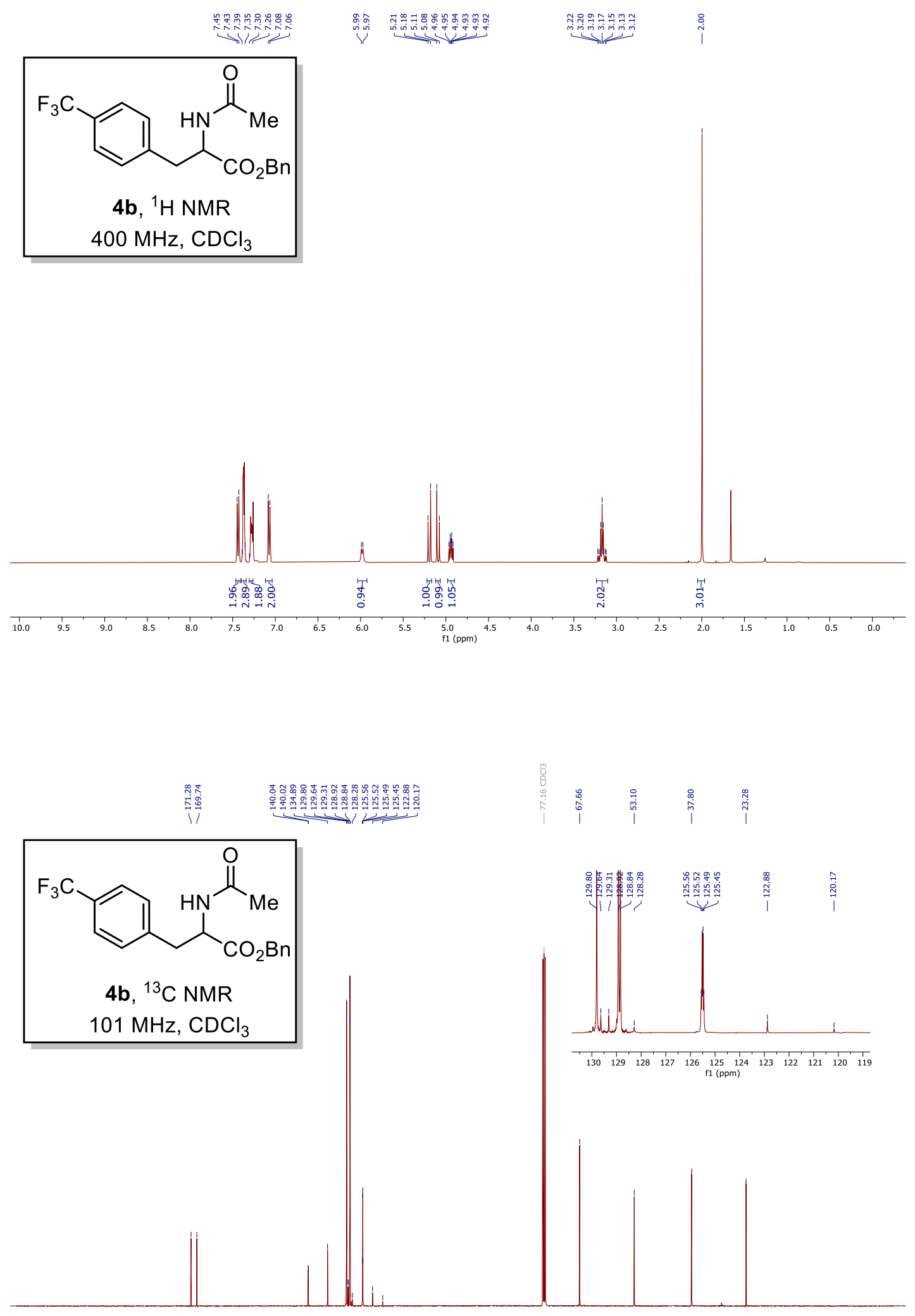


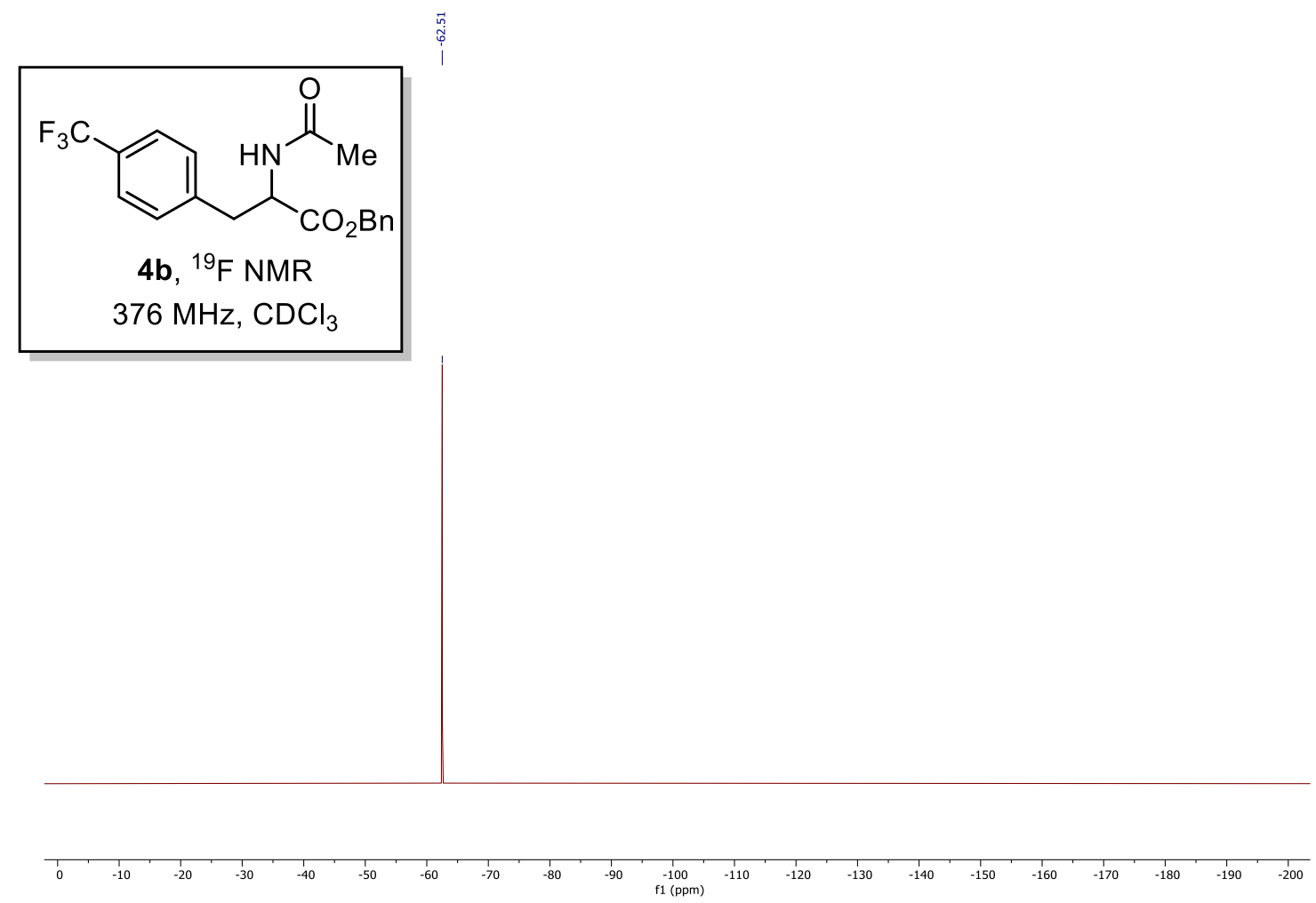



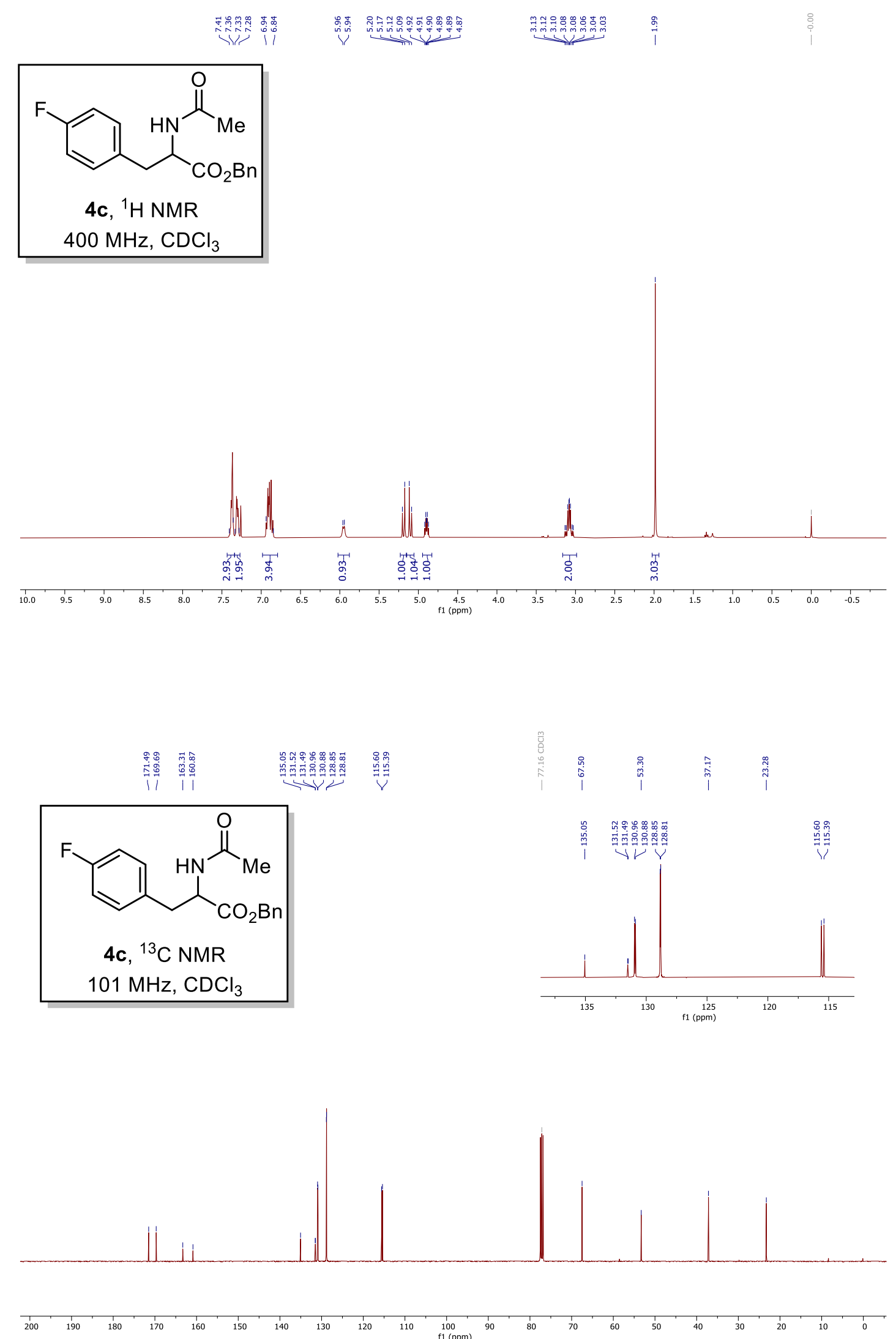

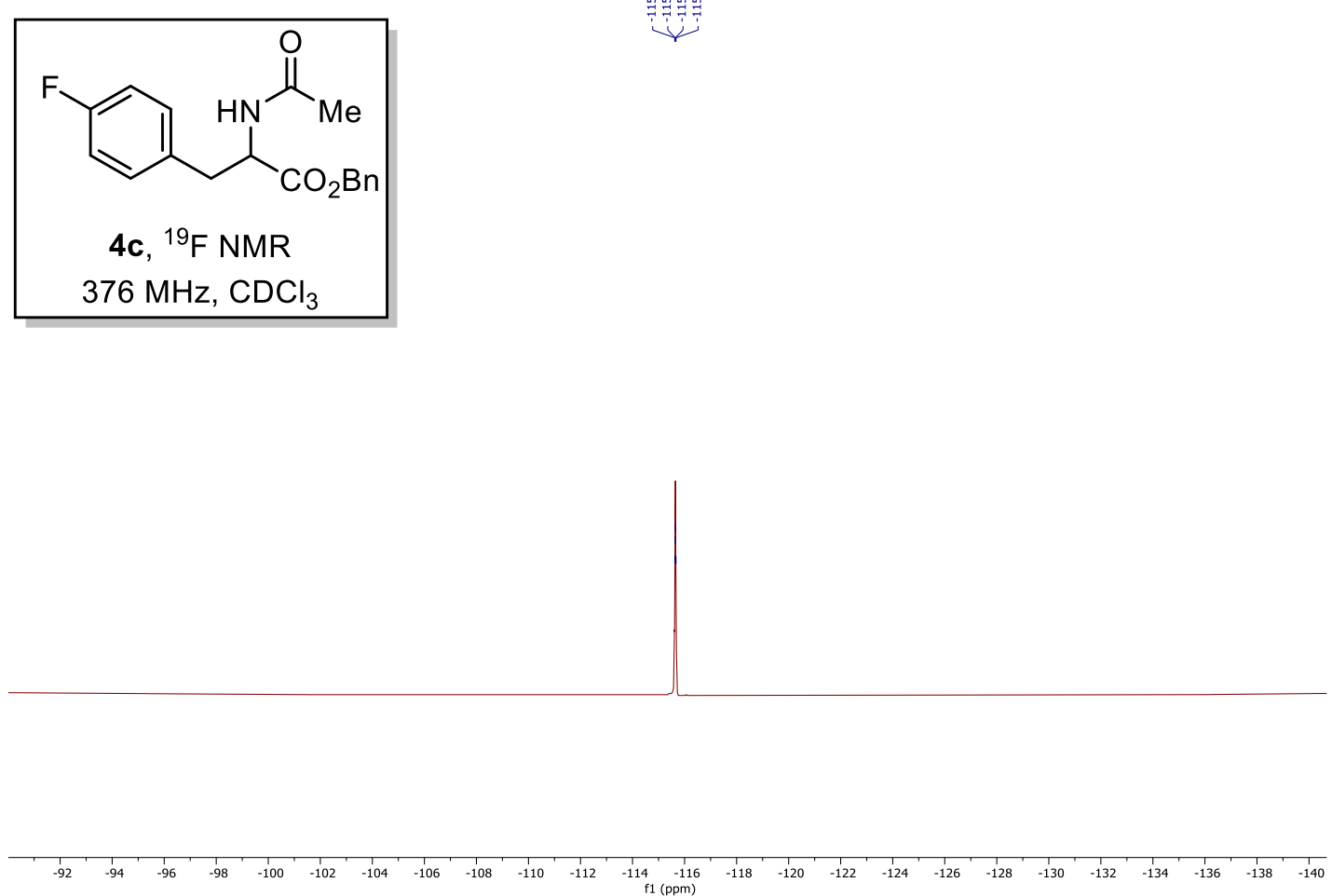

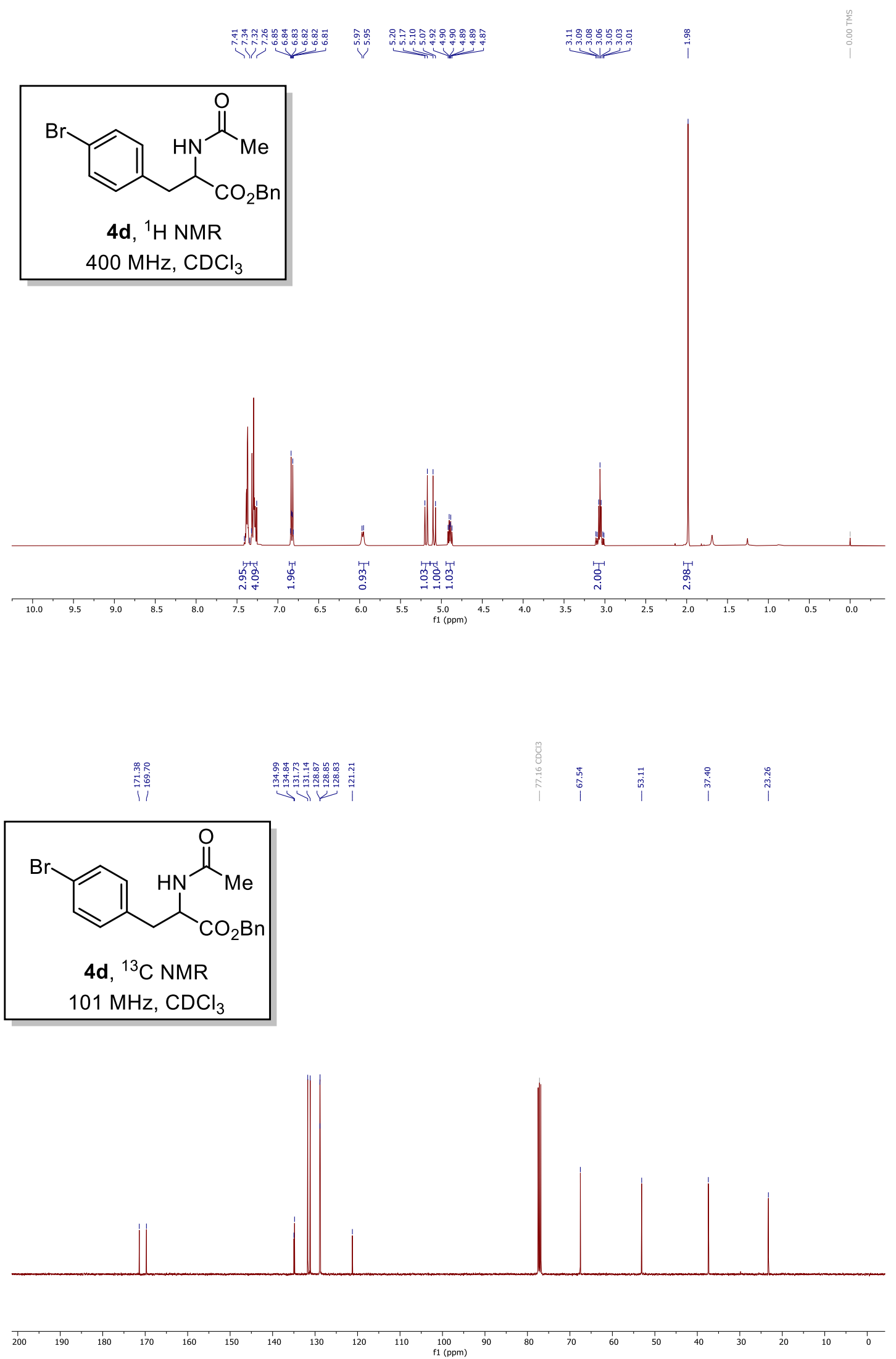

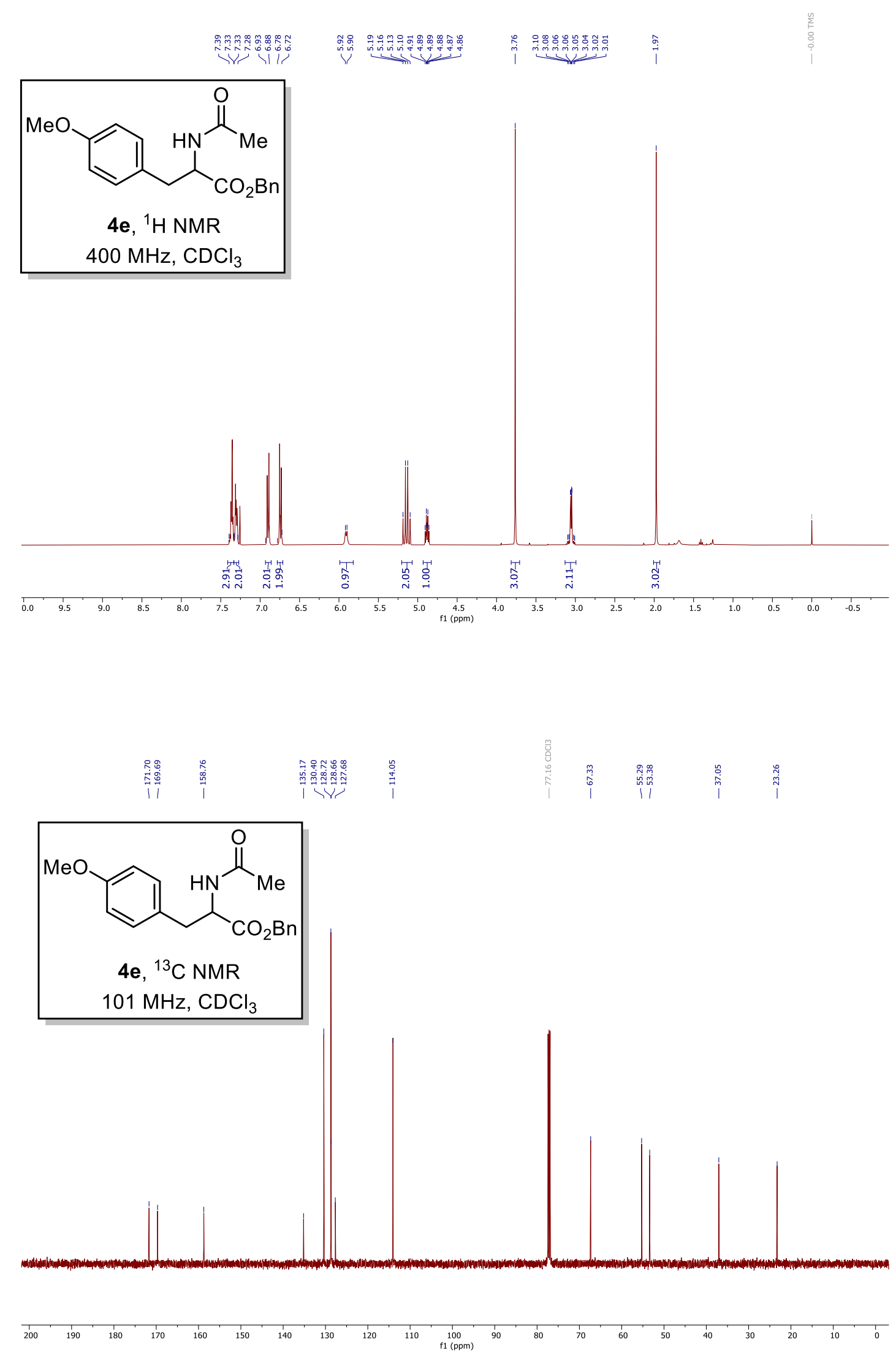


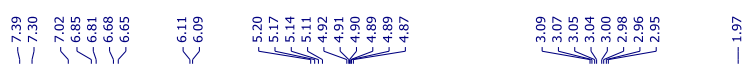

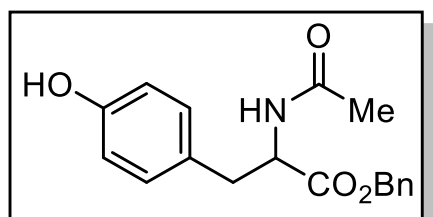

4f, ${ }^{1} \mathrm{H}$ NMR $400 \mathrm{MHz}, \mathrm{CDCl}_{3}$
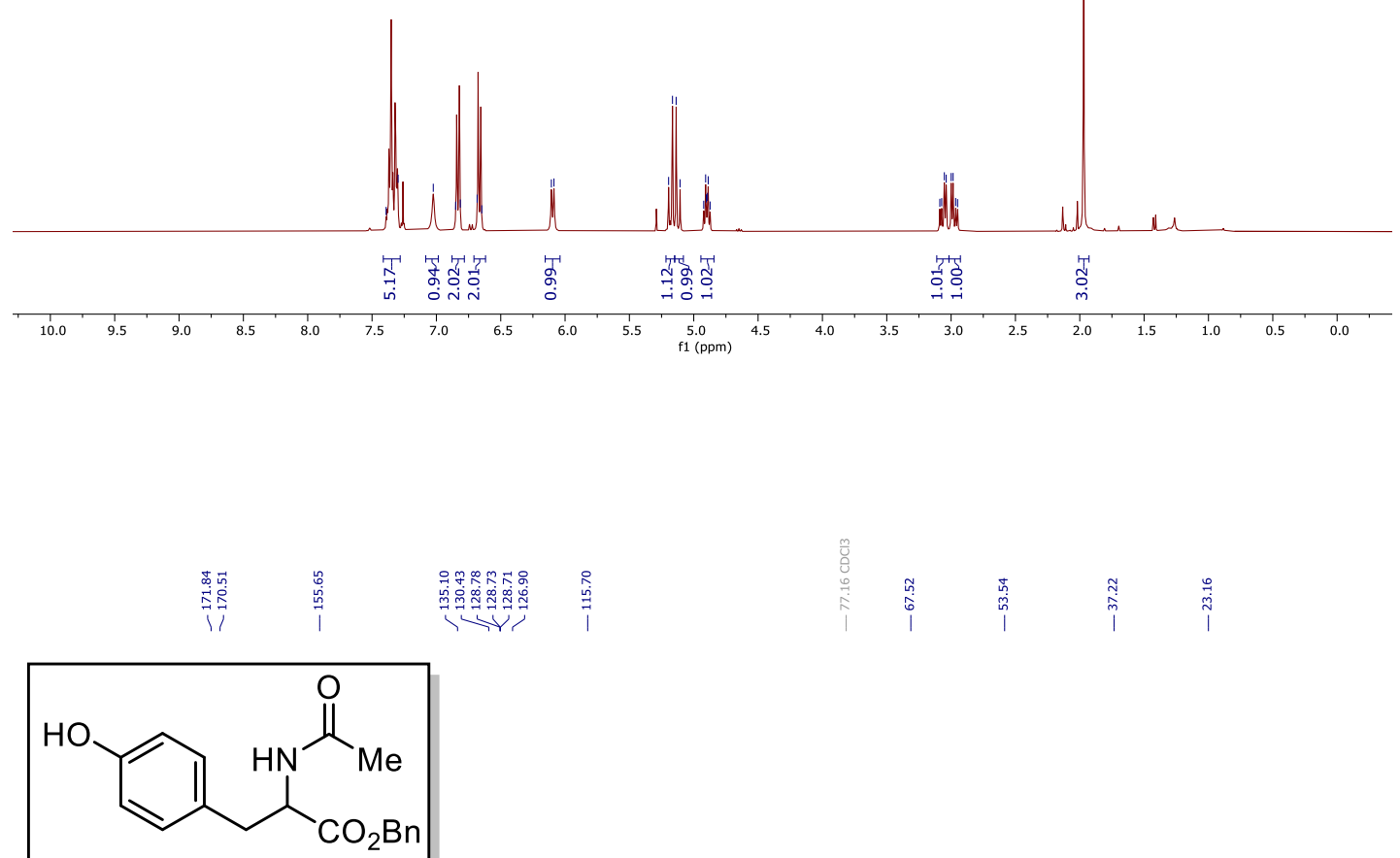

4f, ${ }^{13} \mathrm{C}$ NMR

$101 \mathrm{MHz}, \mathrm{CDCl}_{3}$

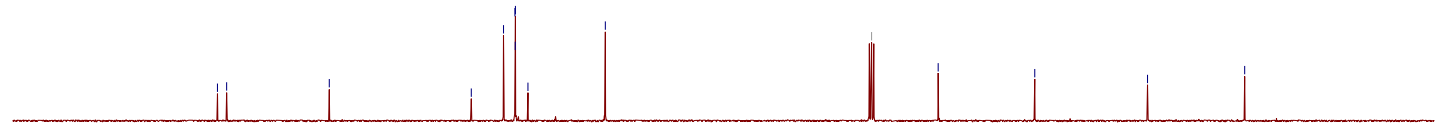

200

100
$f 1(p p m)$ 

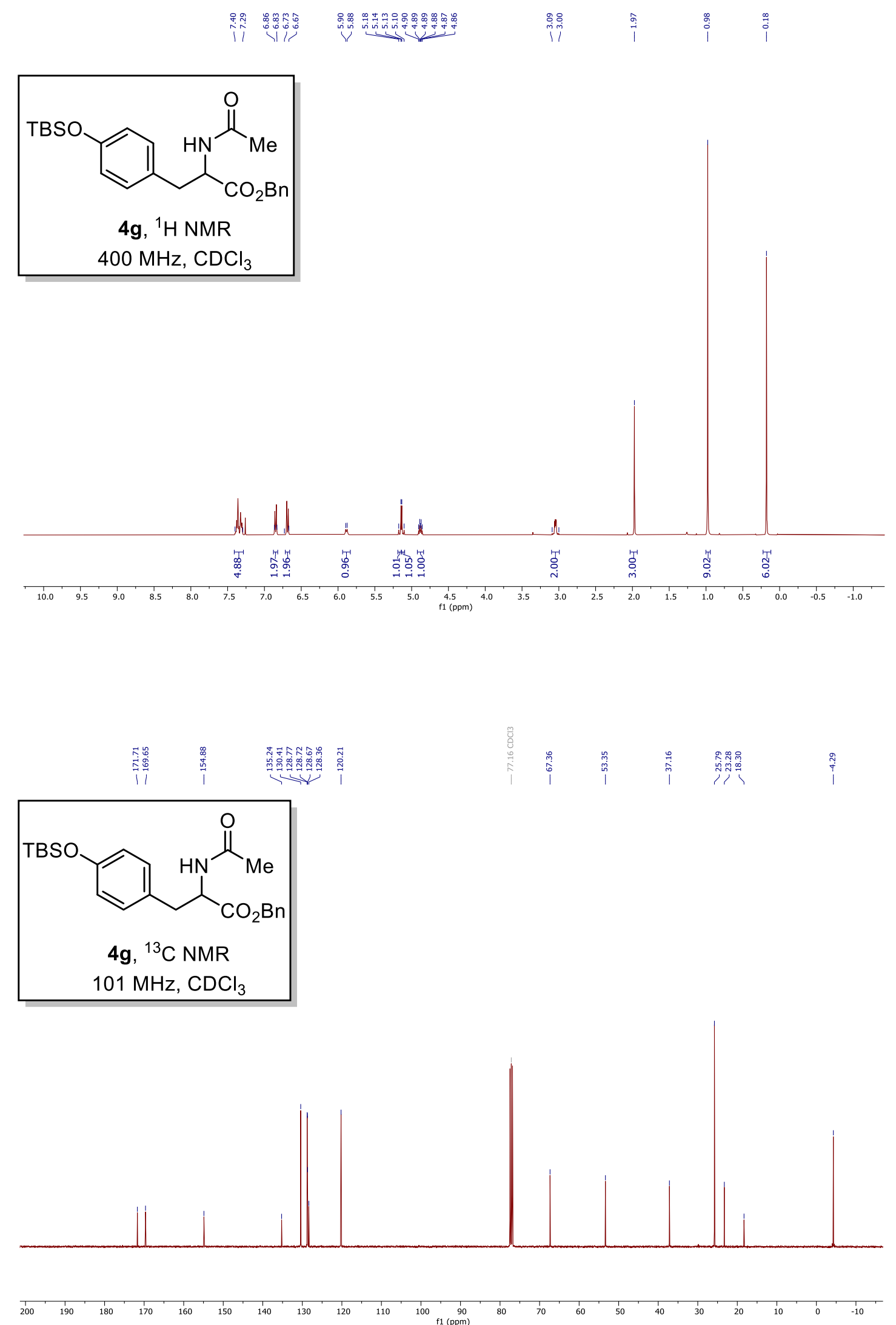
<smiles>C=Cc1ccc(CC(NC(C)=O)C(=O)OCc2ccccc2)cc1</smiles>

4h, ${ }^{1} \mathrm{H}$ NMR $400 \mathrm{MHz}, \mathrm{CDCl}_{3}$
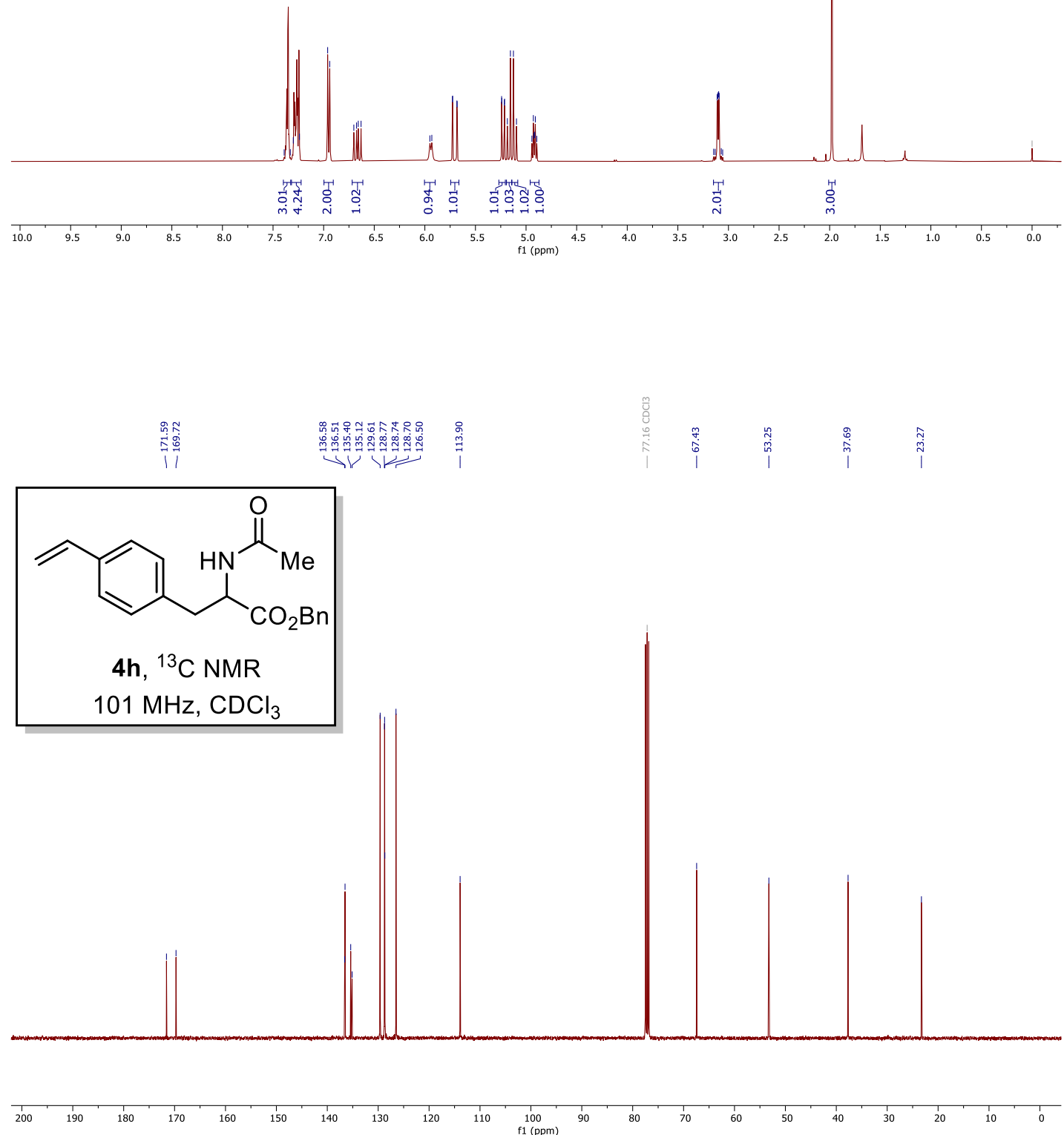

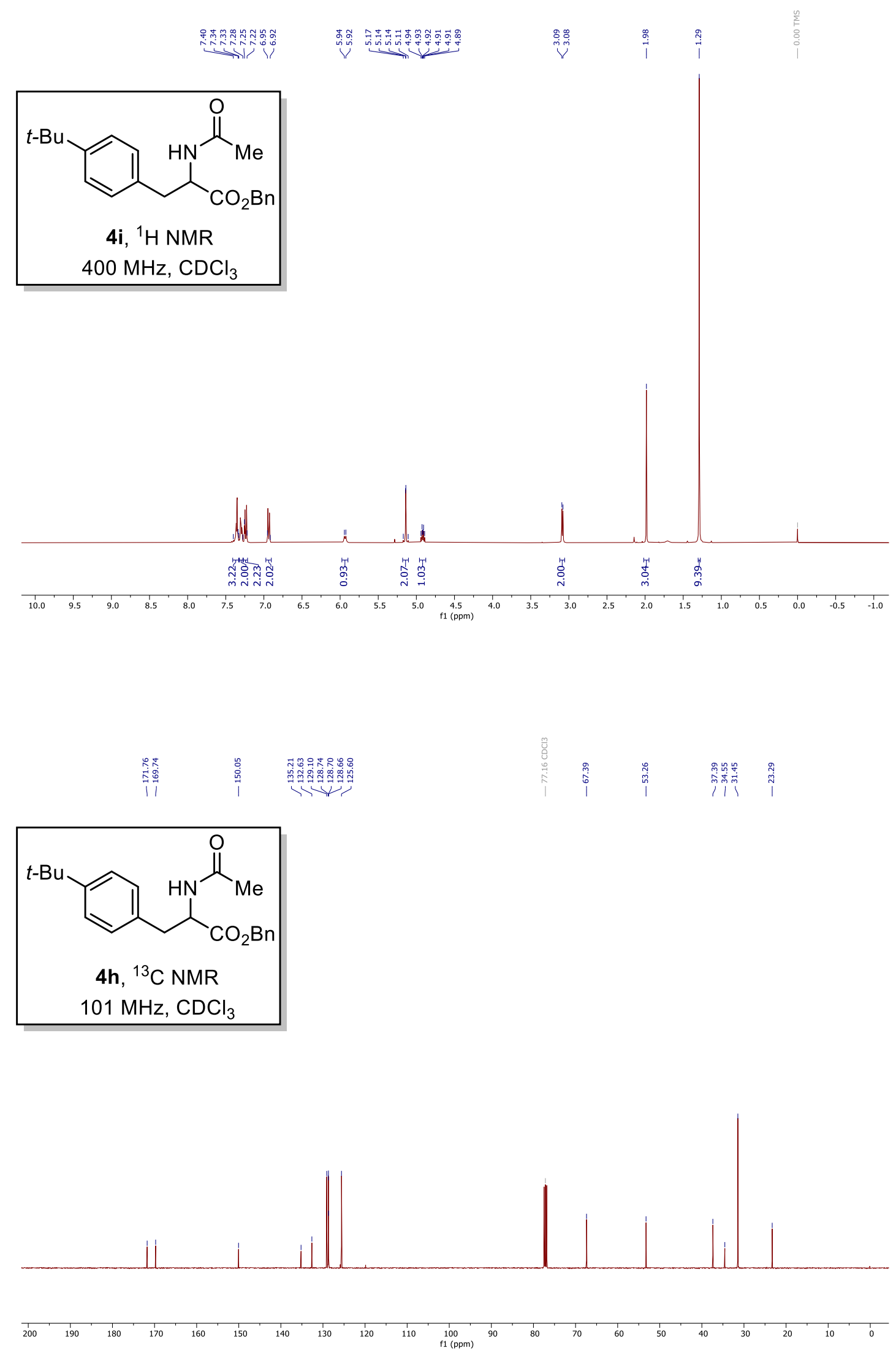

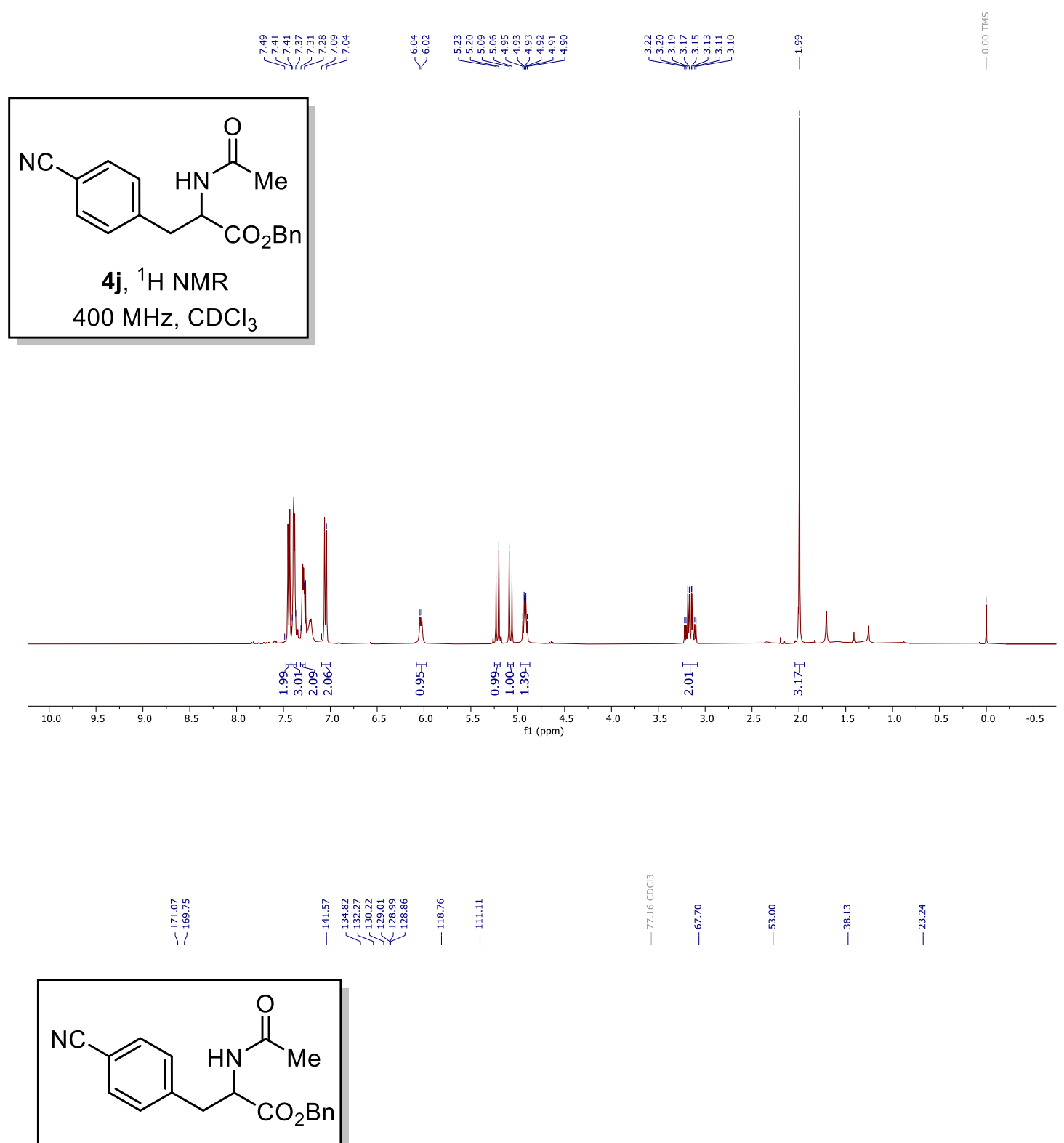

4j, ${ }^{13} \mathrm{C}$ NMR

$101 \mathrm{MHz}, \mathrm{CDCl}_{3}$
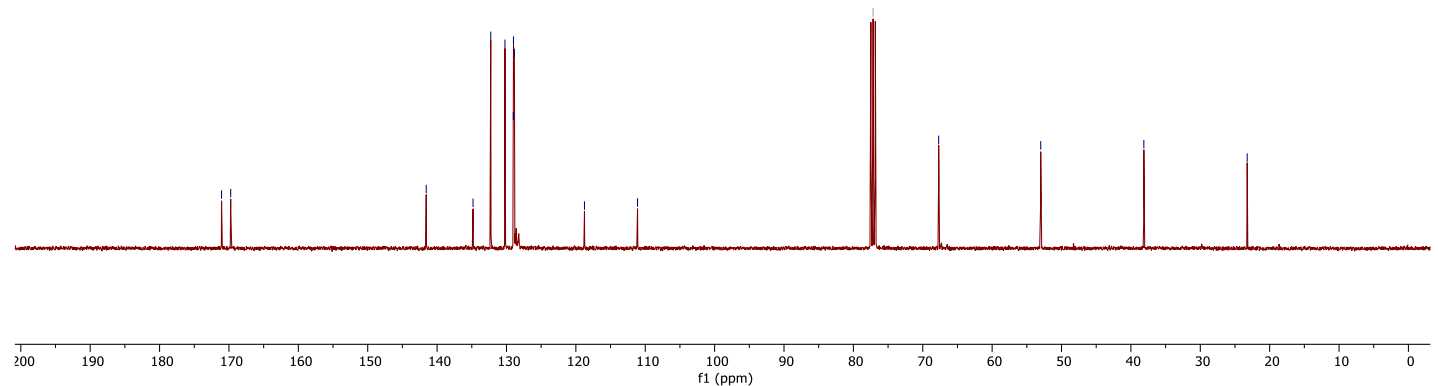

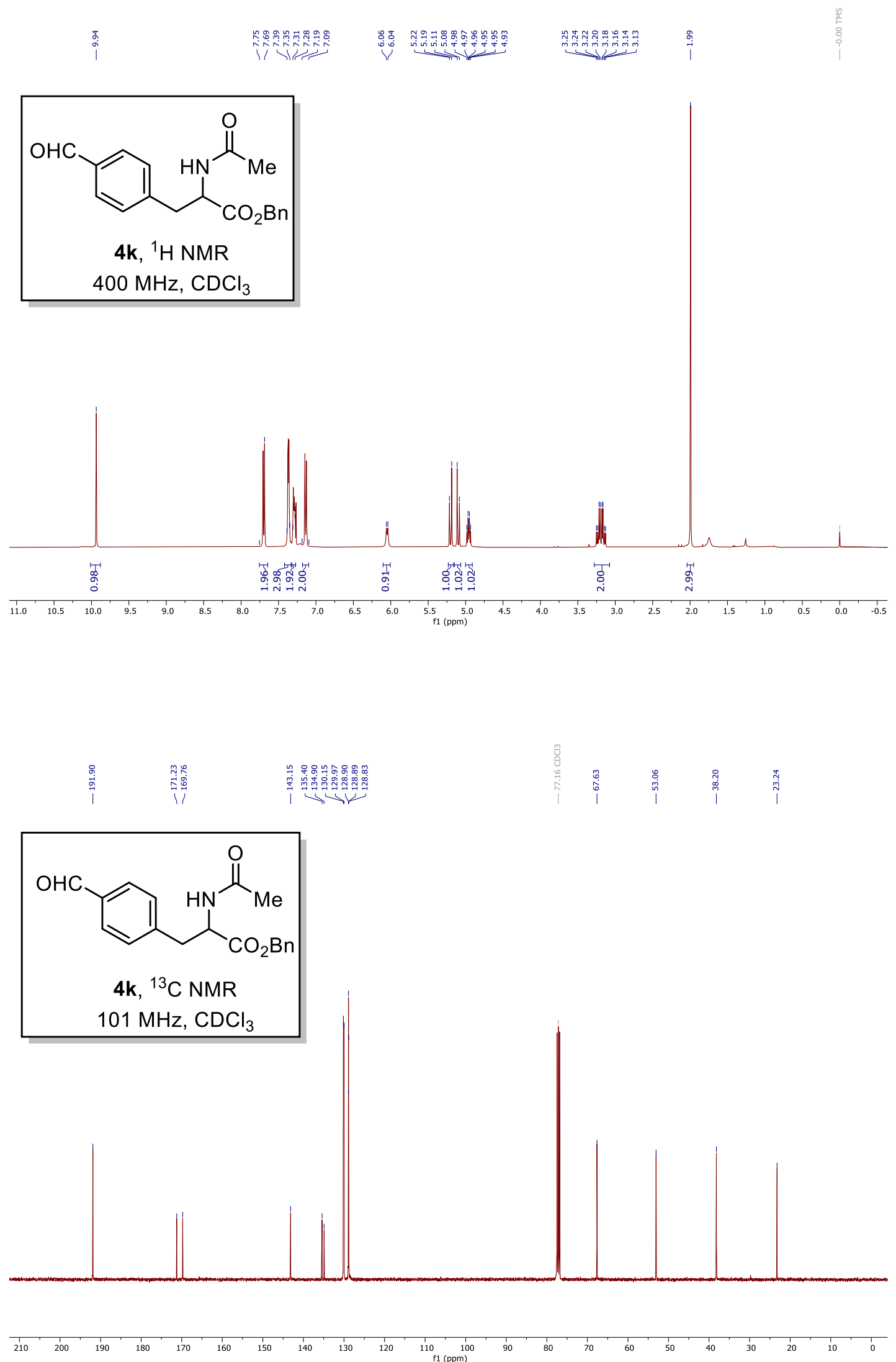

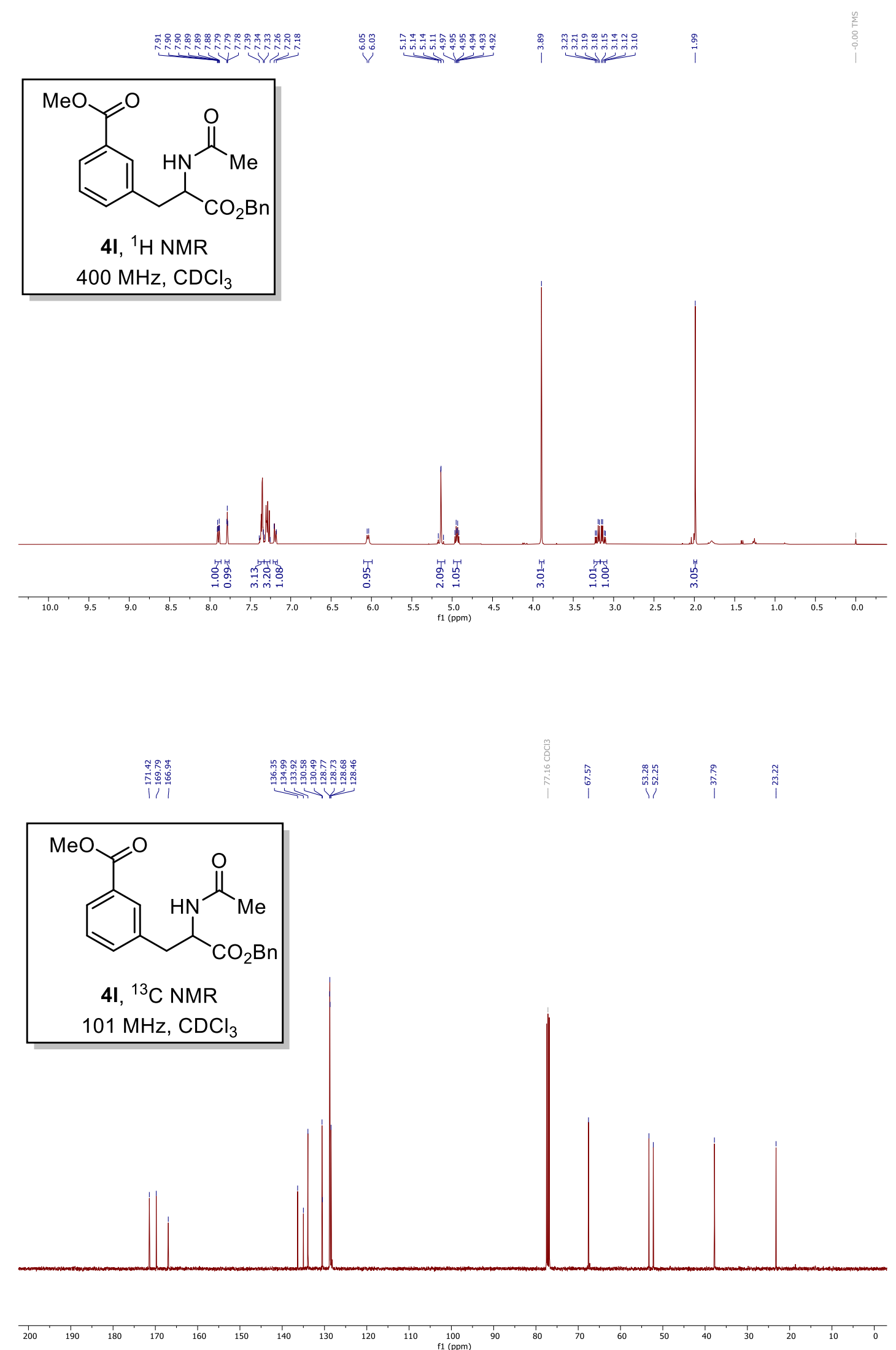

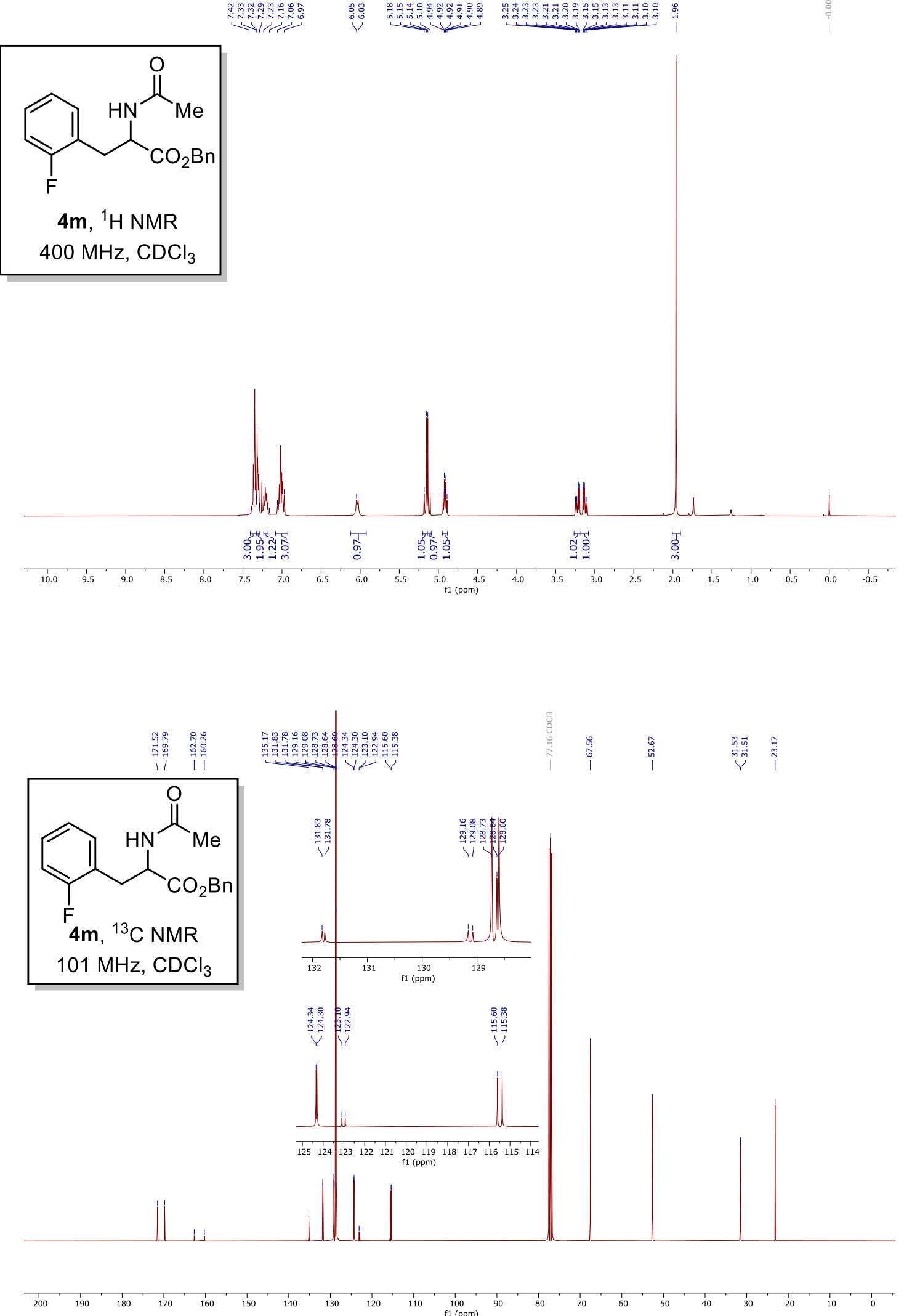


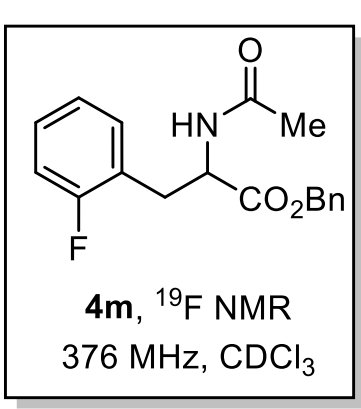

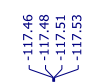

$4 \mathrm{~m},{ }^{19} \mathrm{~F}$ NMR

376 $\mathrm{MHz}, \mathrm{CDCl}_{3}$

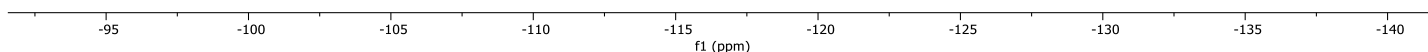



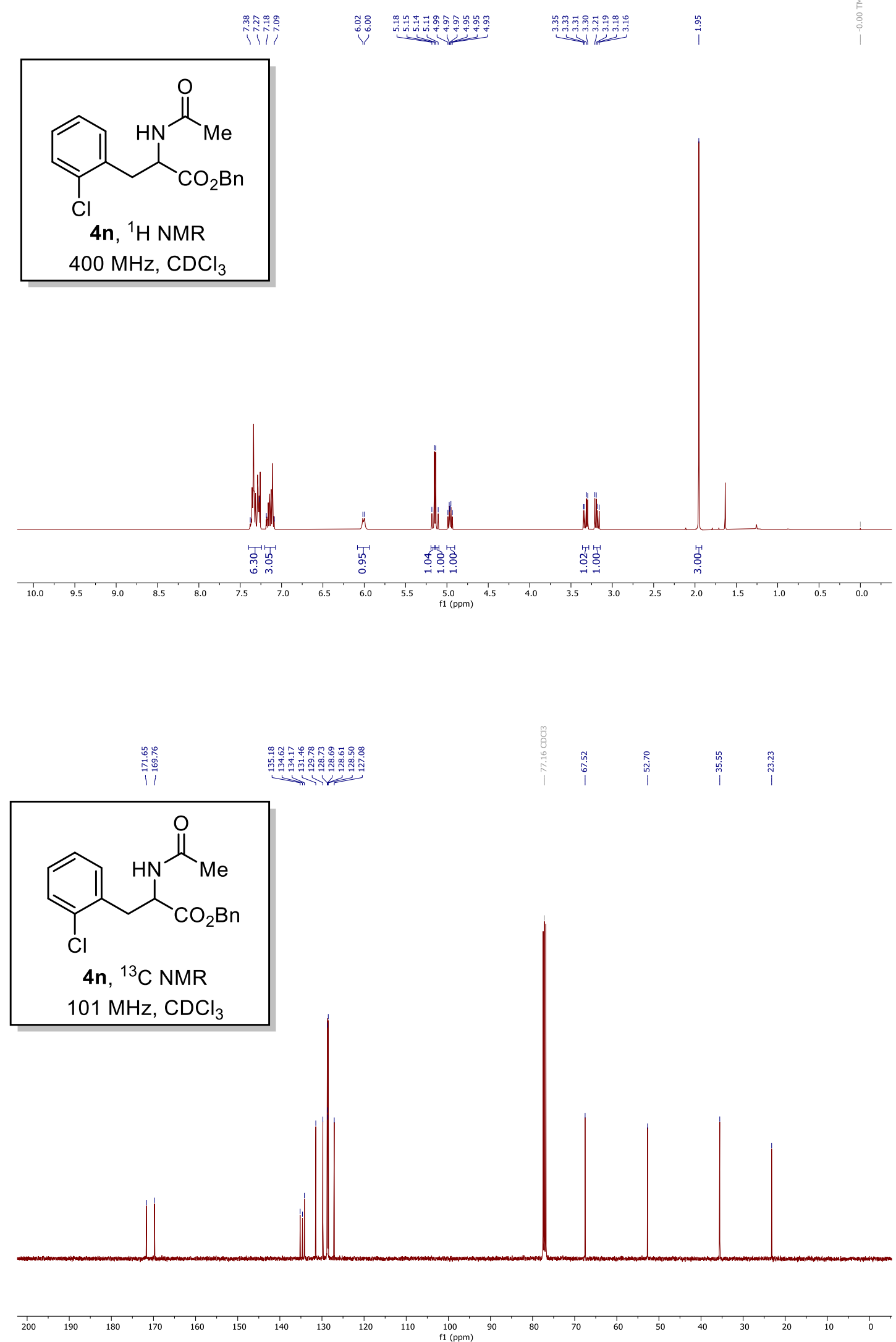

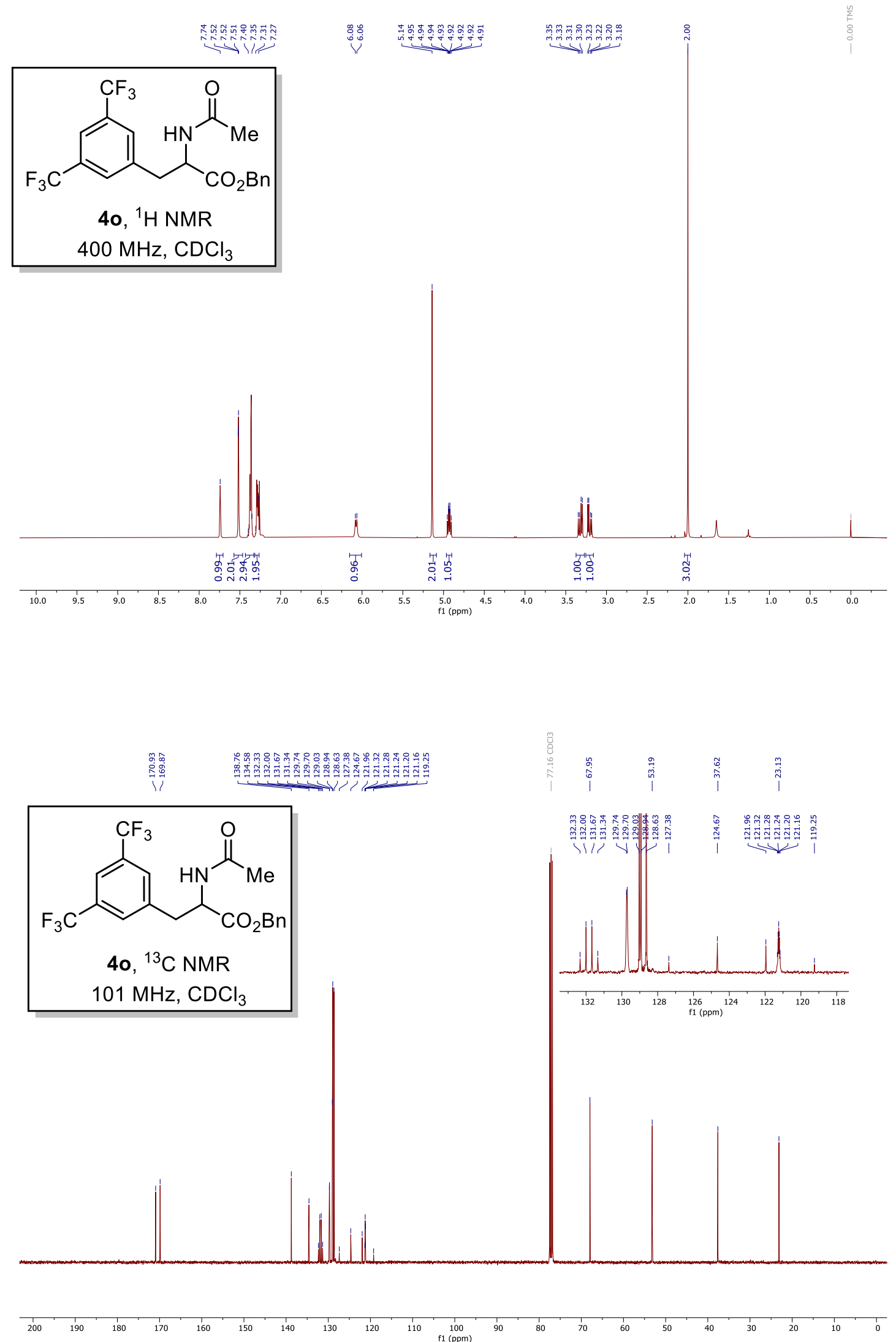


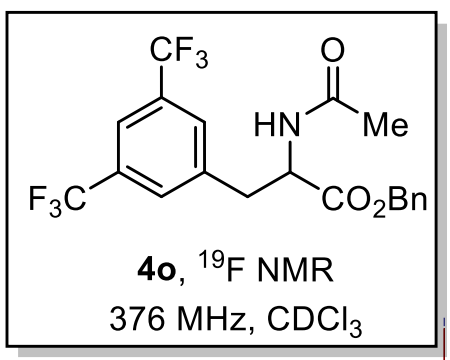

T
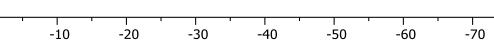

1
-100
$f 1(\mathrm{ppm})$ 

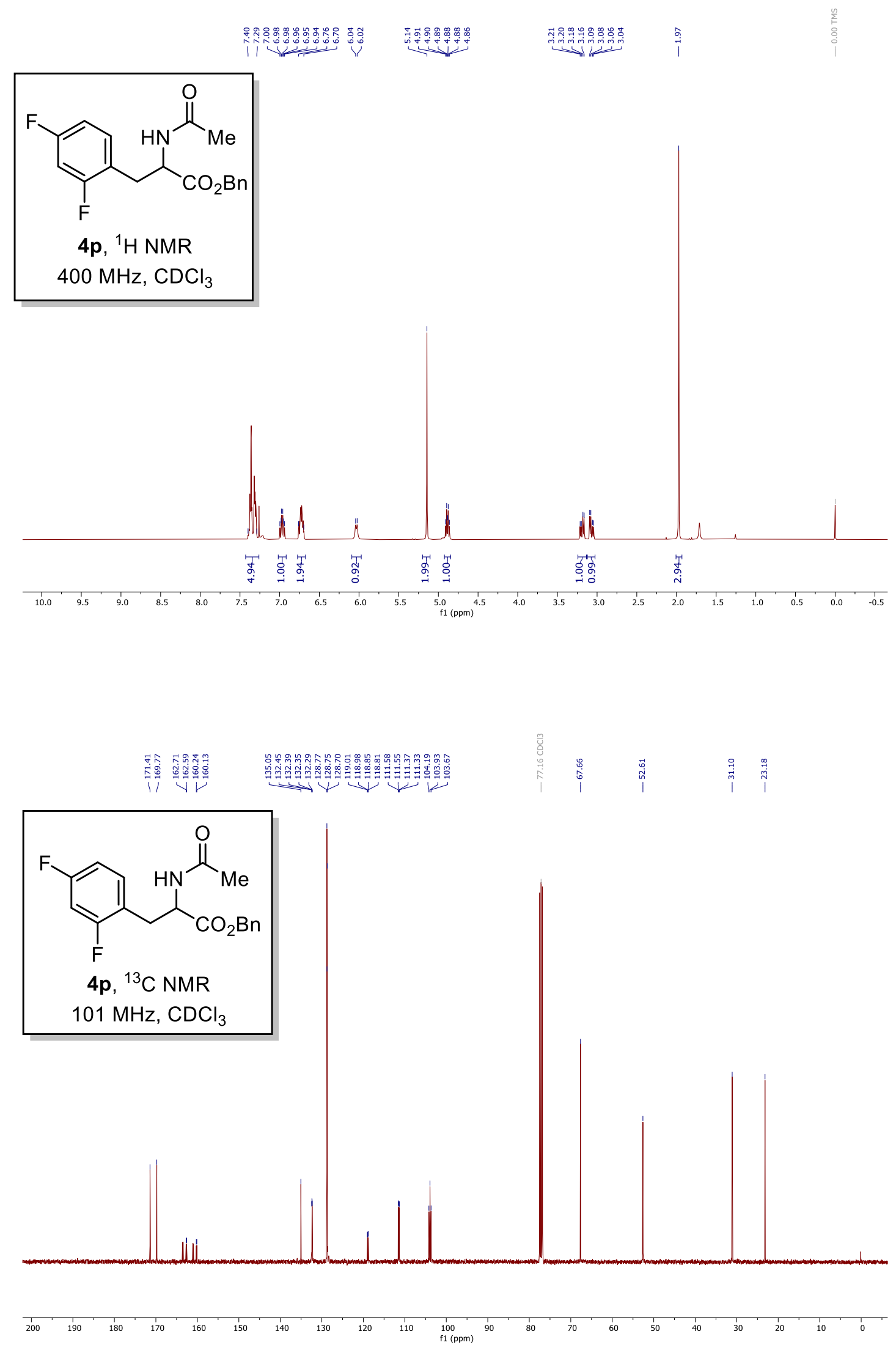


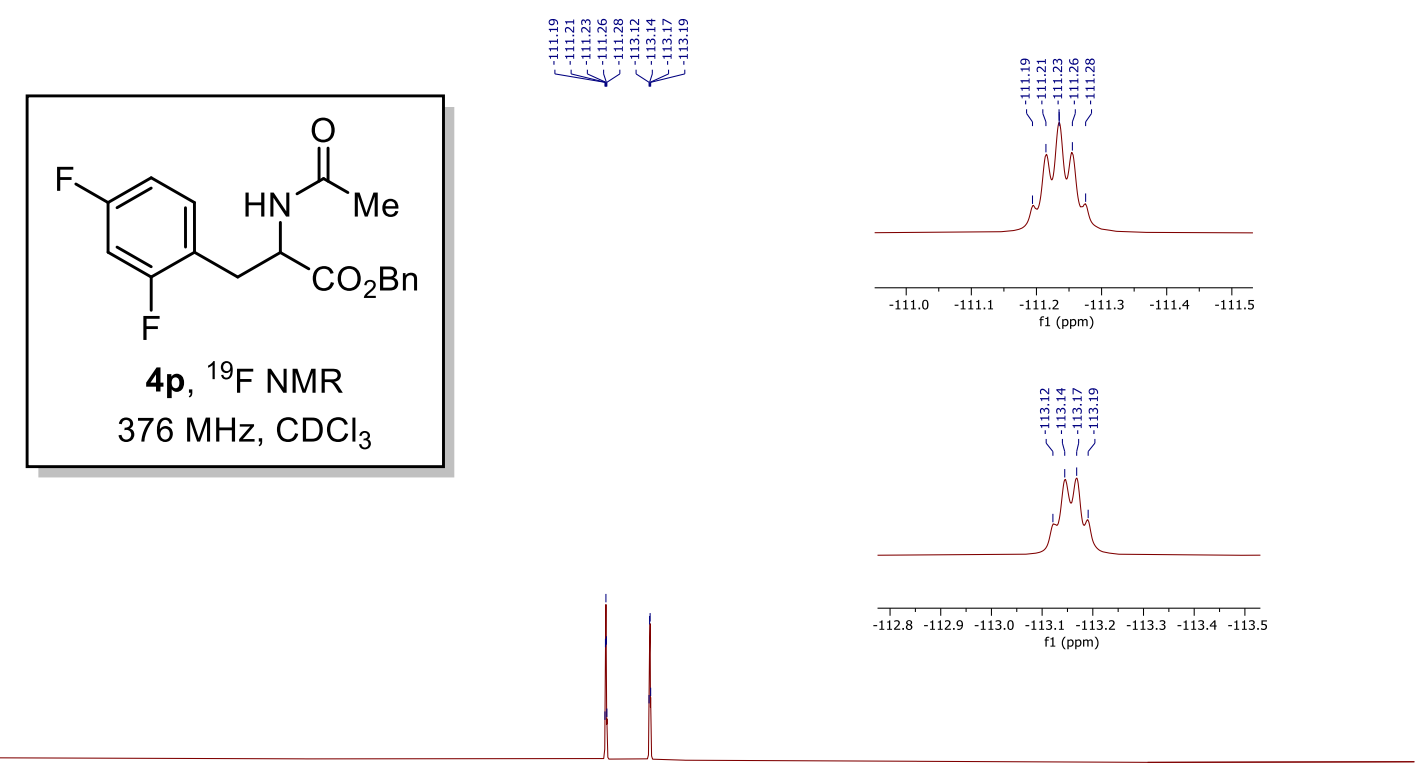

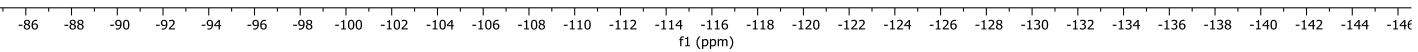



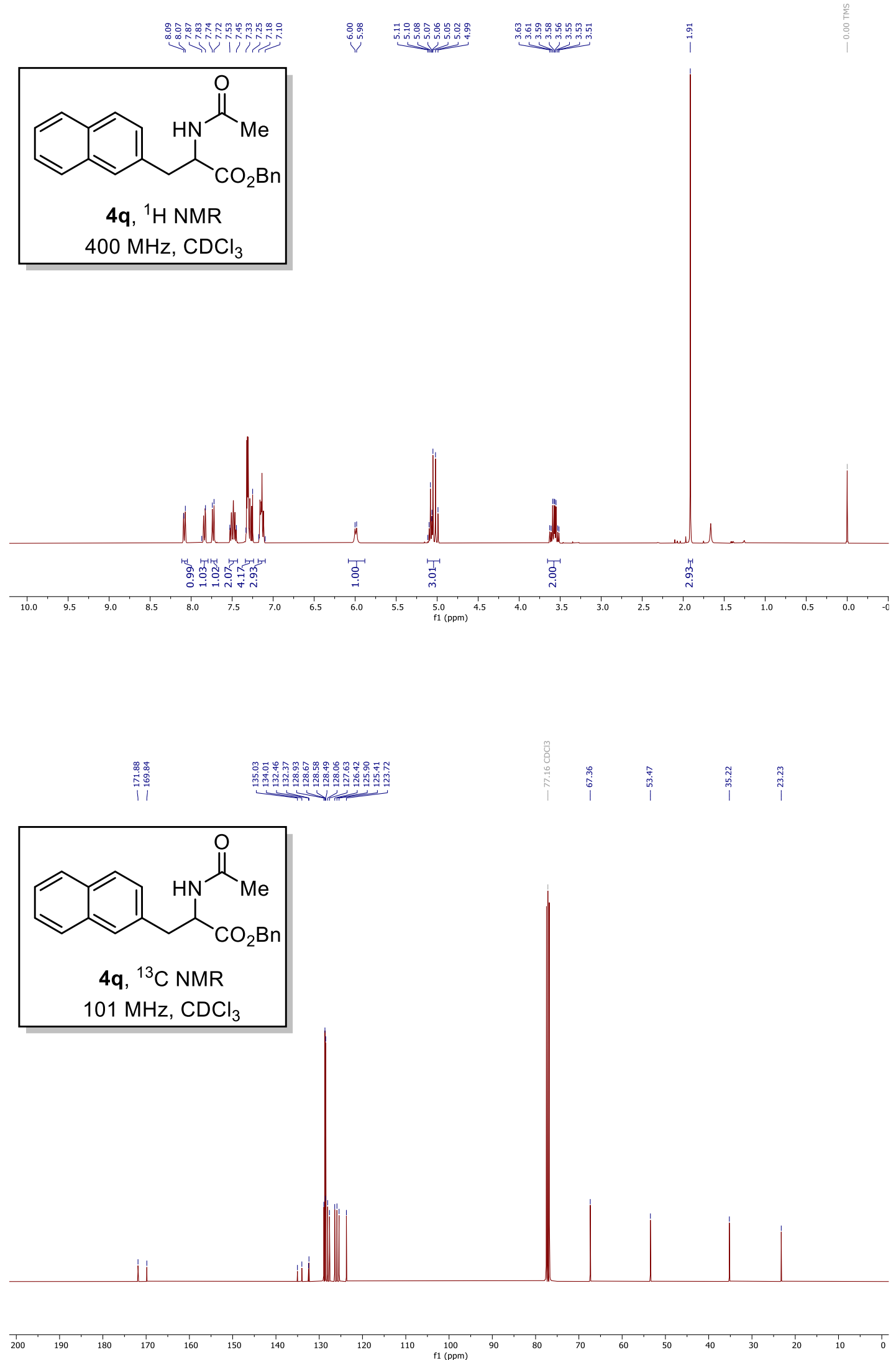


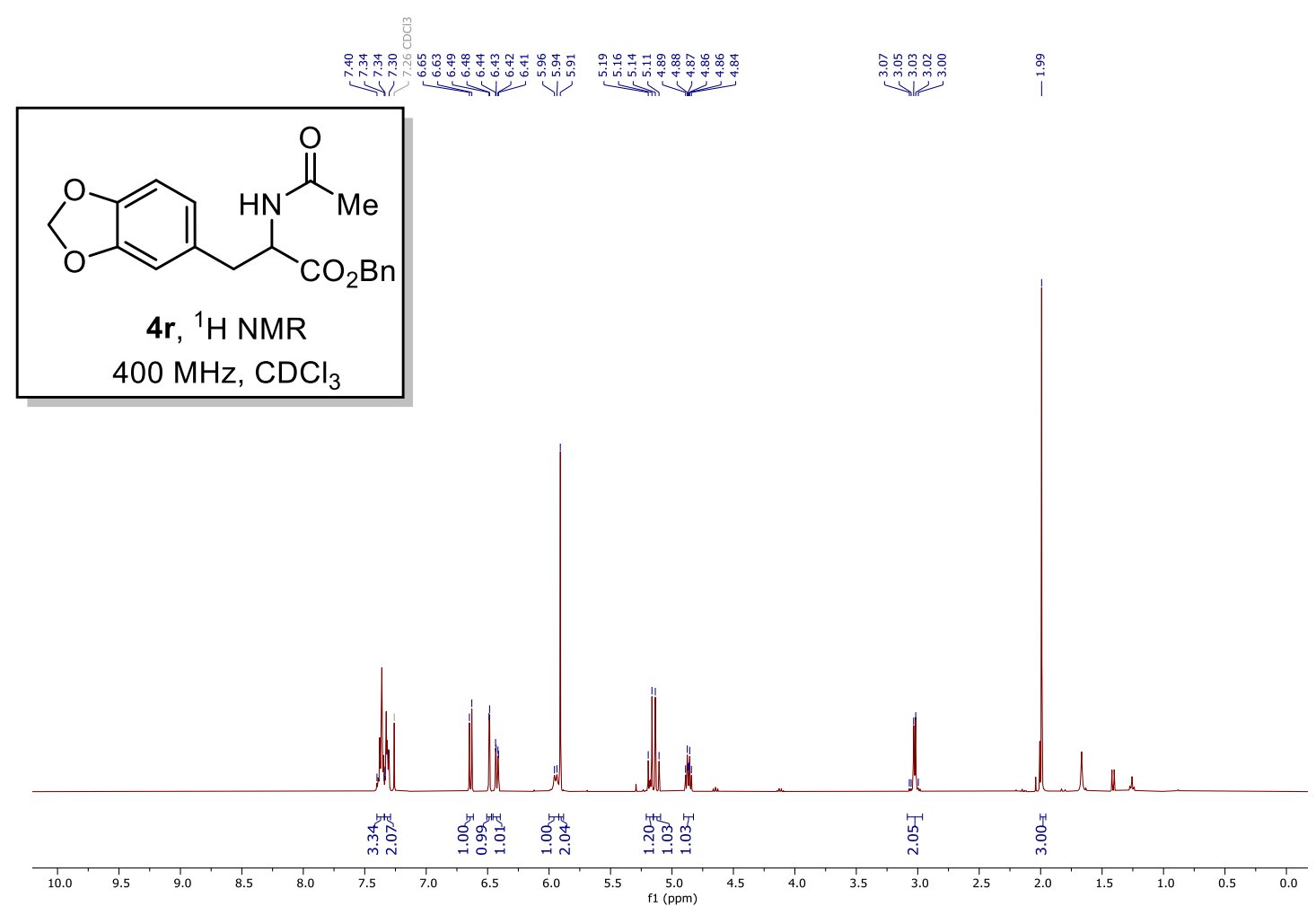

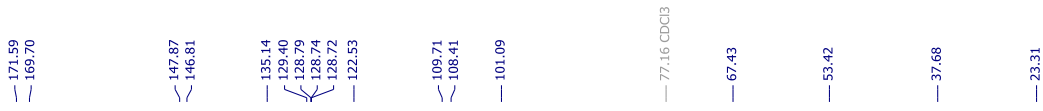

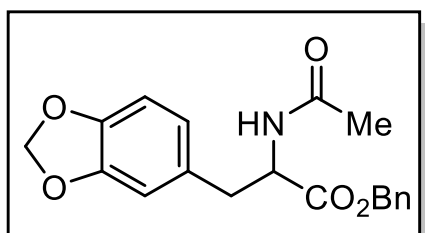

4r, ${ }^{13} \mathrm{C}$ NMR

$101 \mathrm{MHz}, \mathrm{CDCl}_{3}$

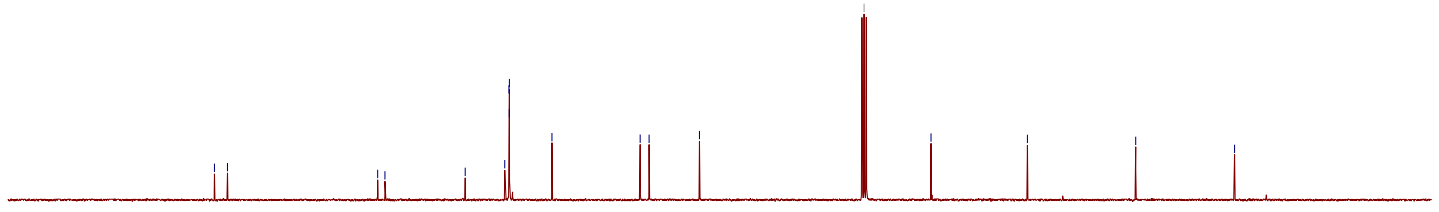

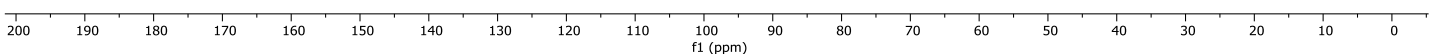



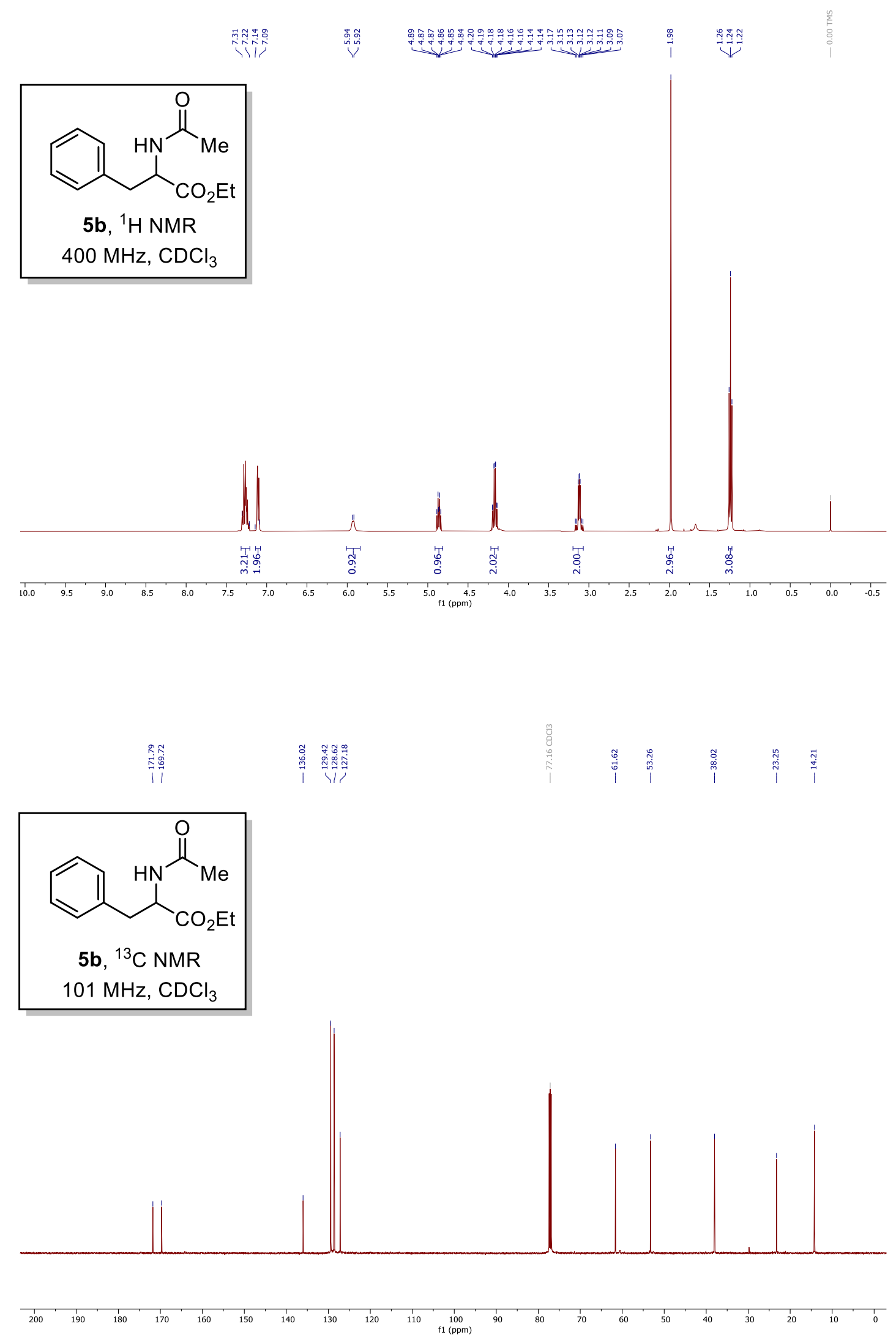

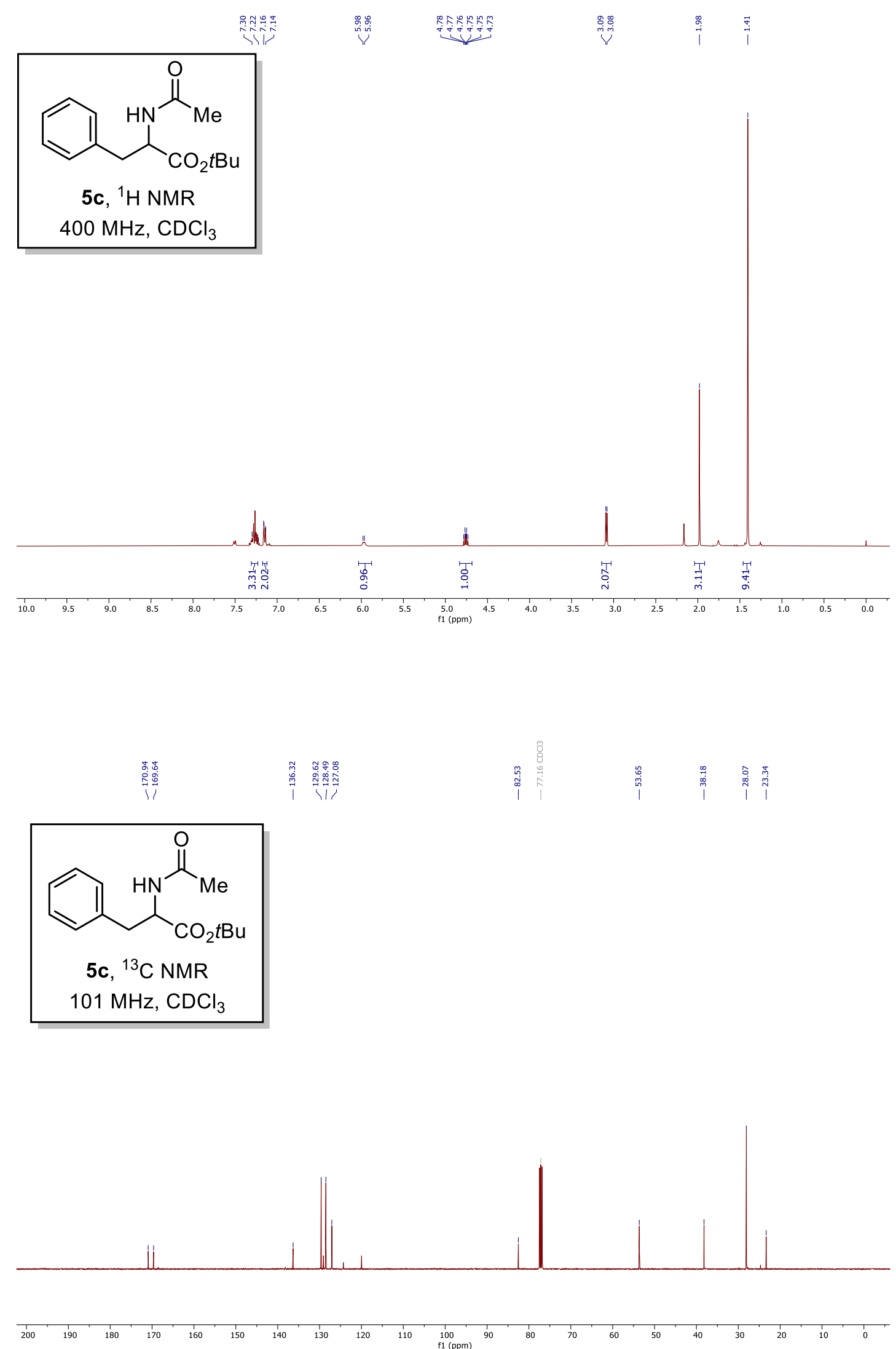

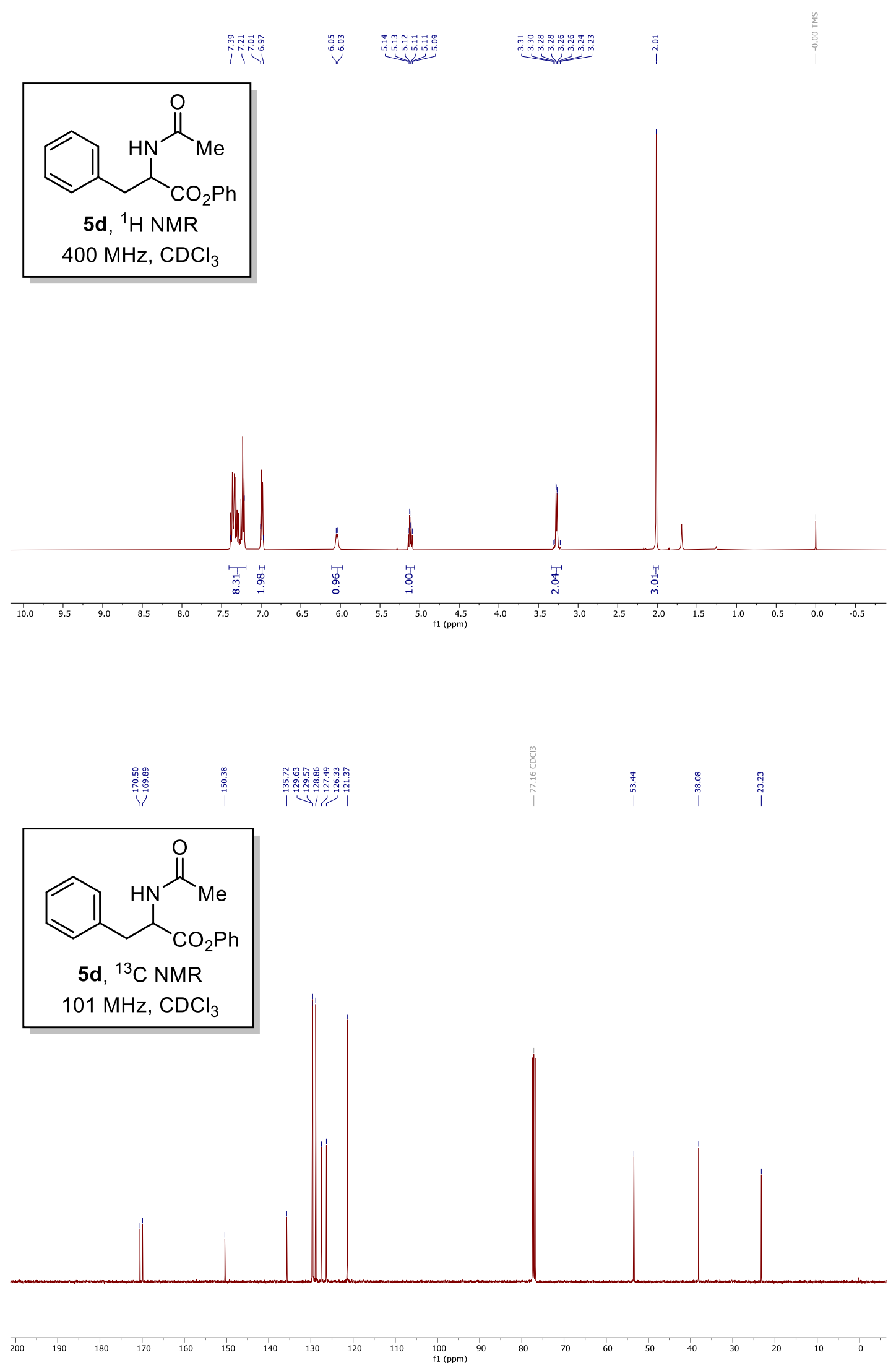

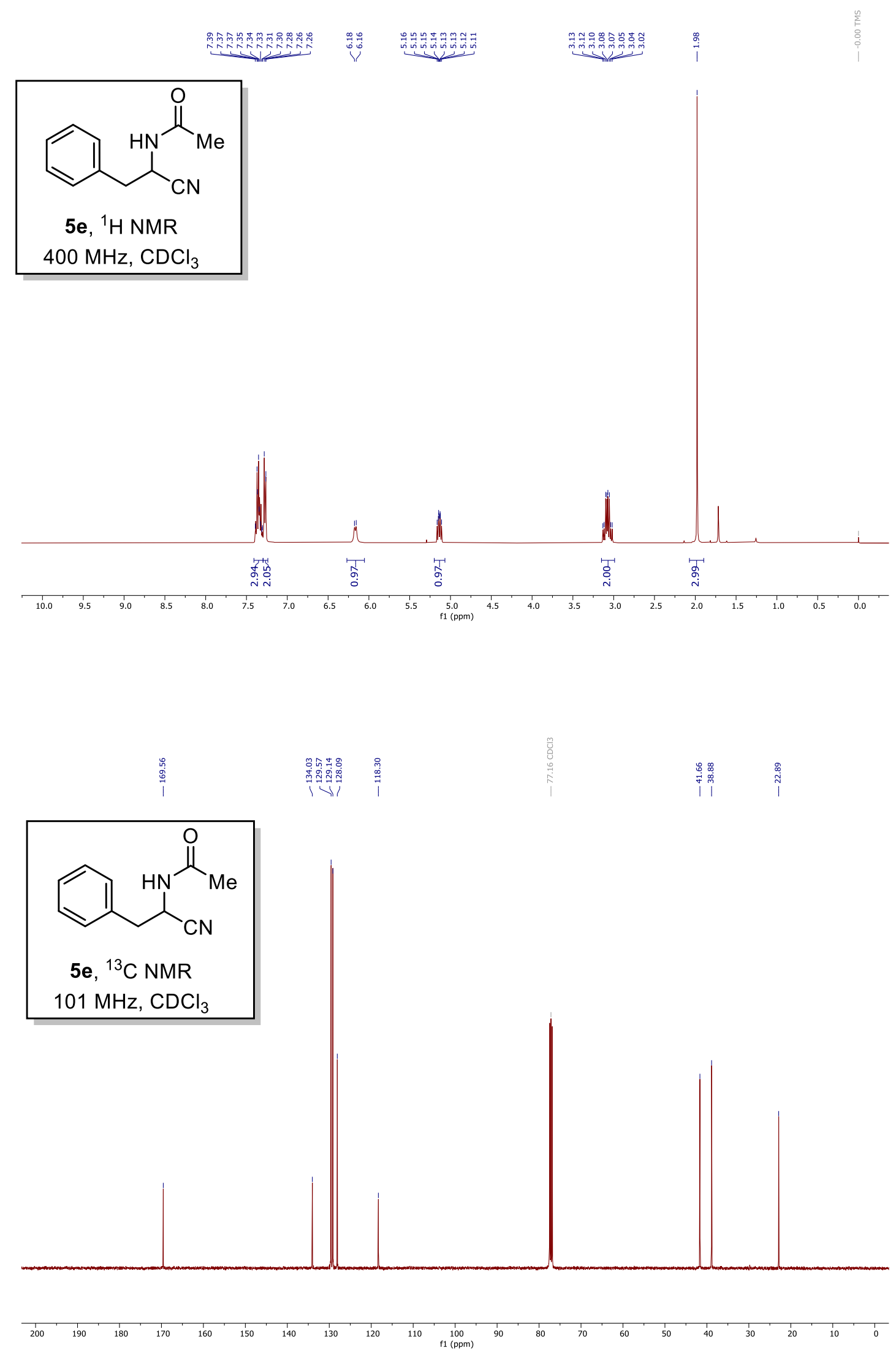

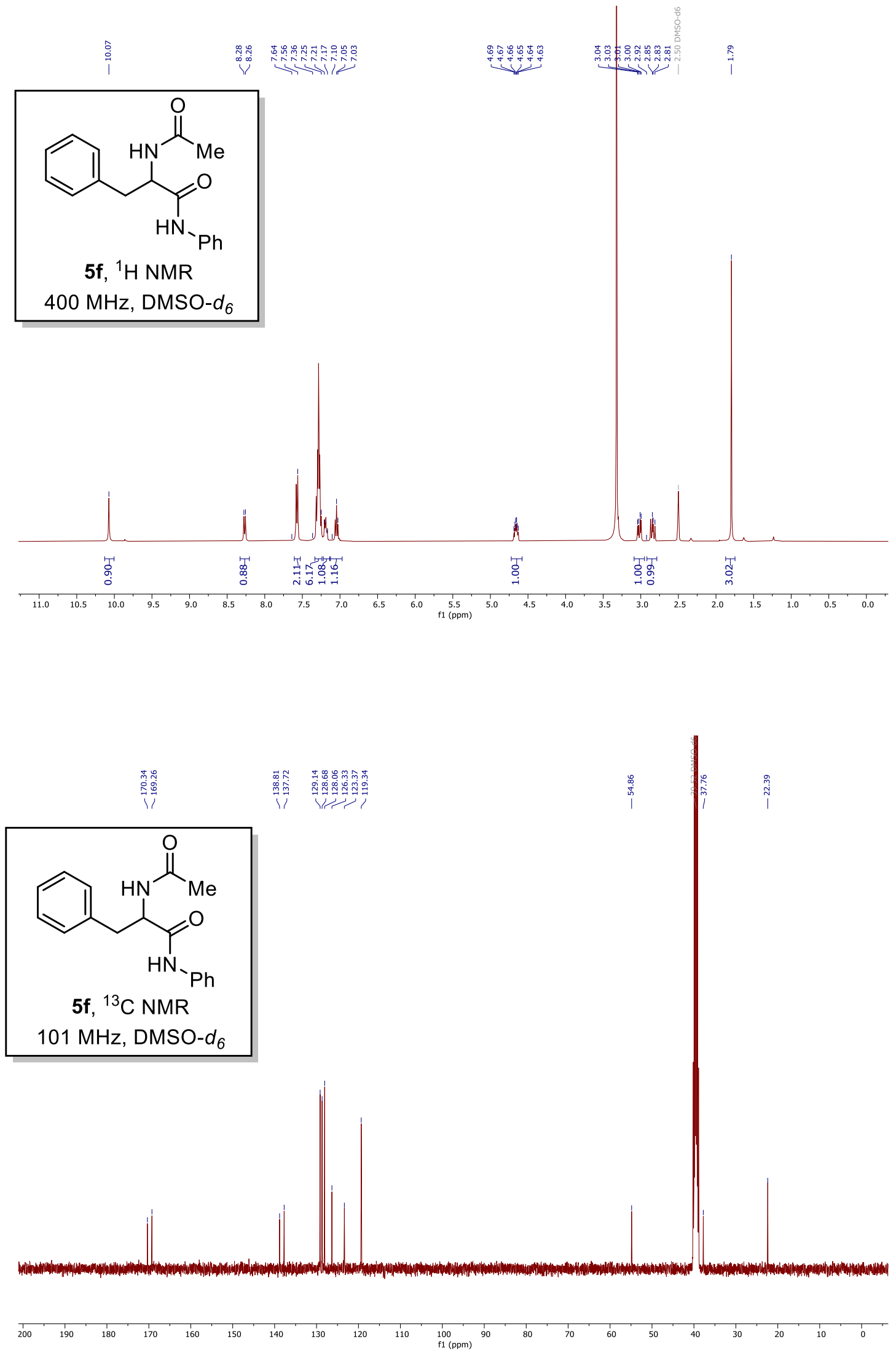

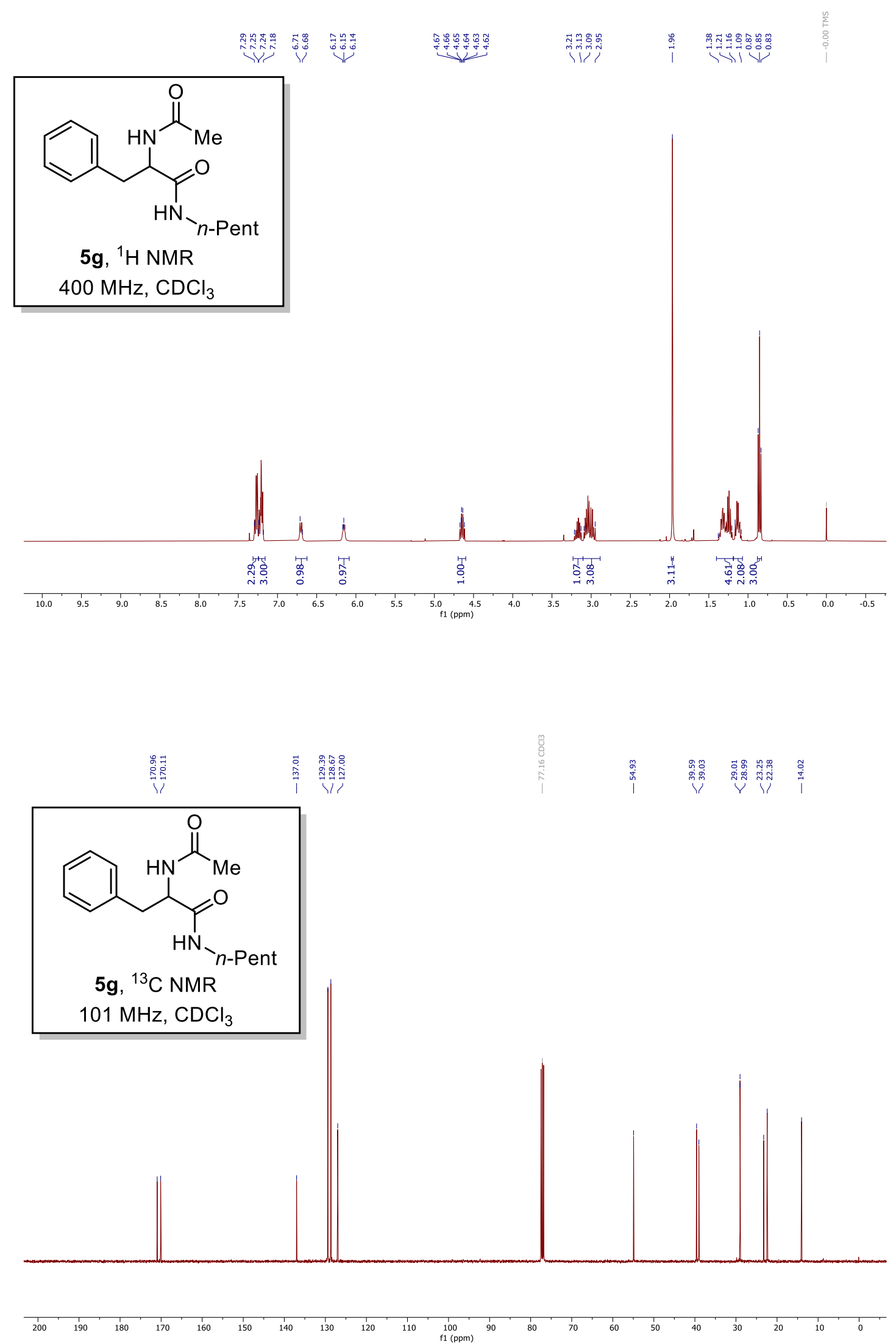

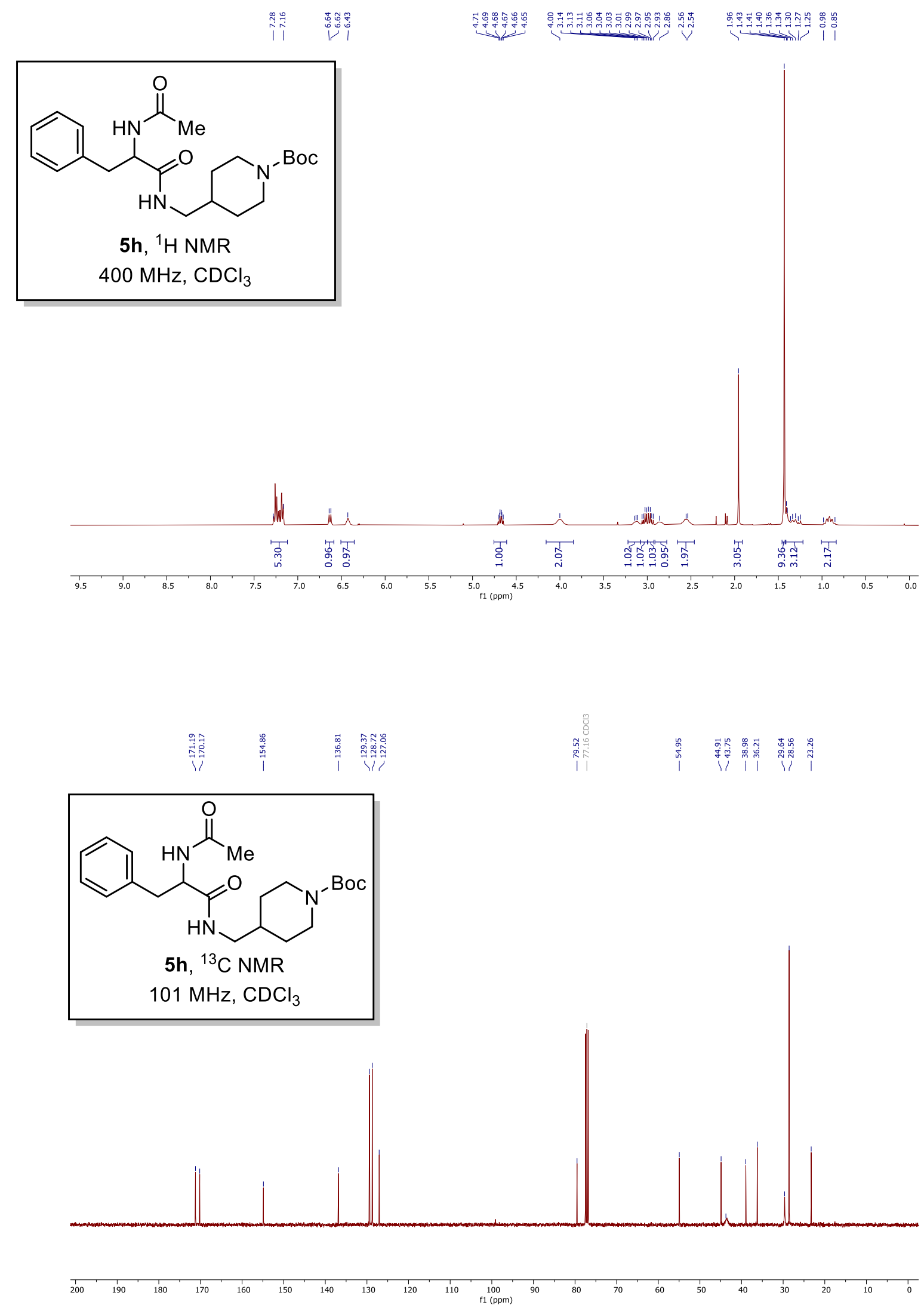

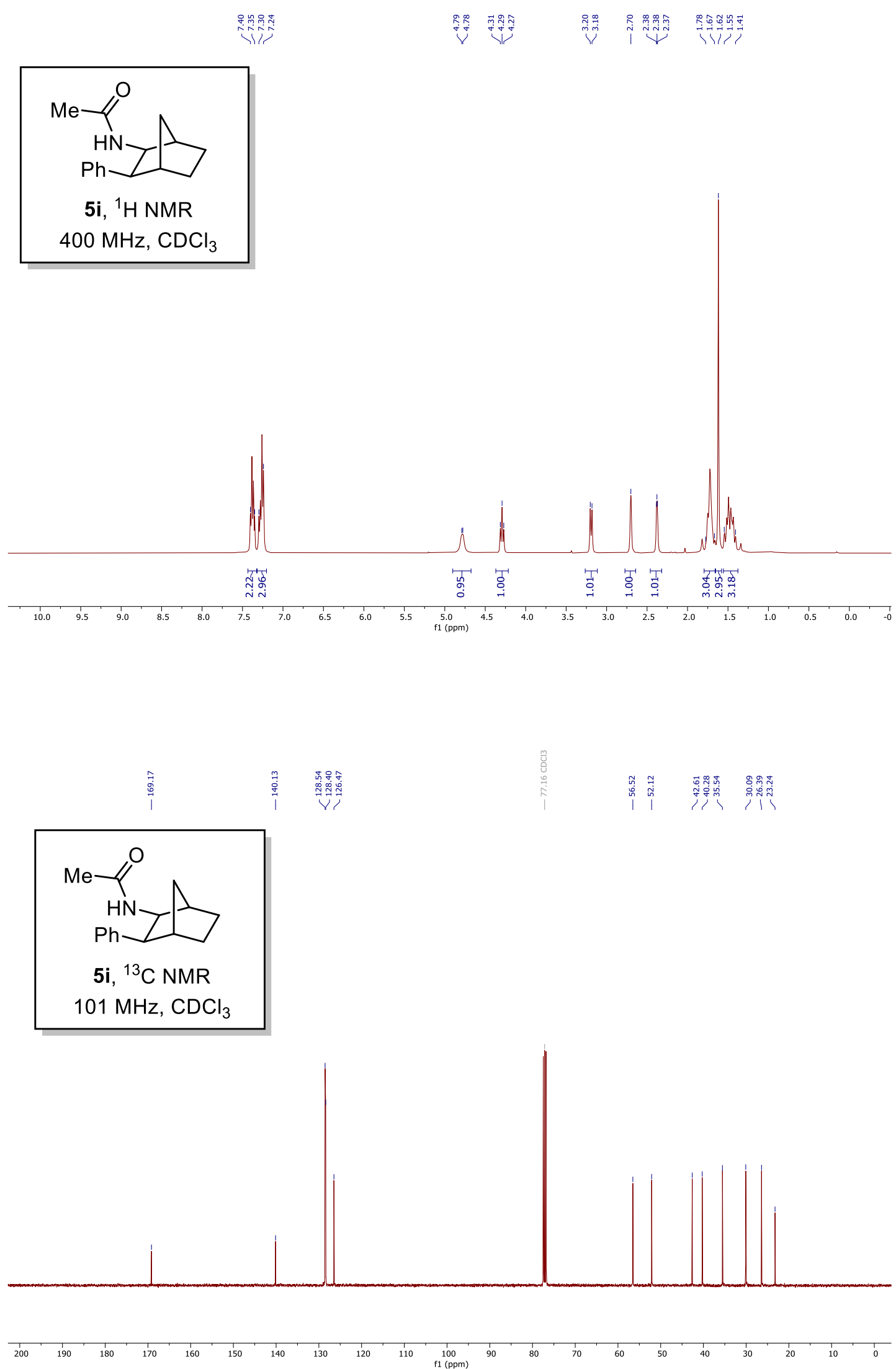

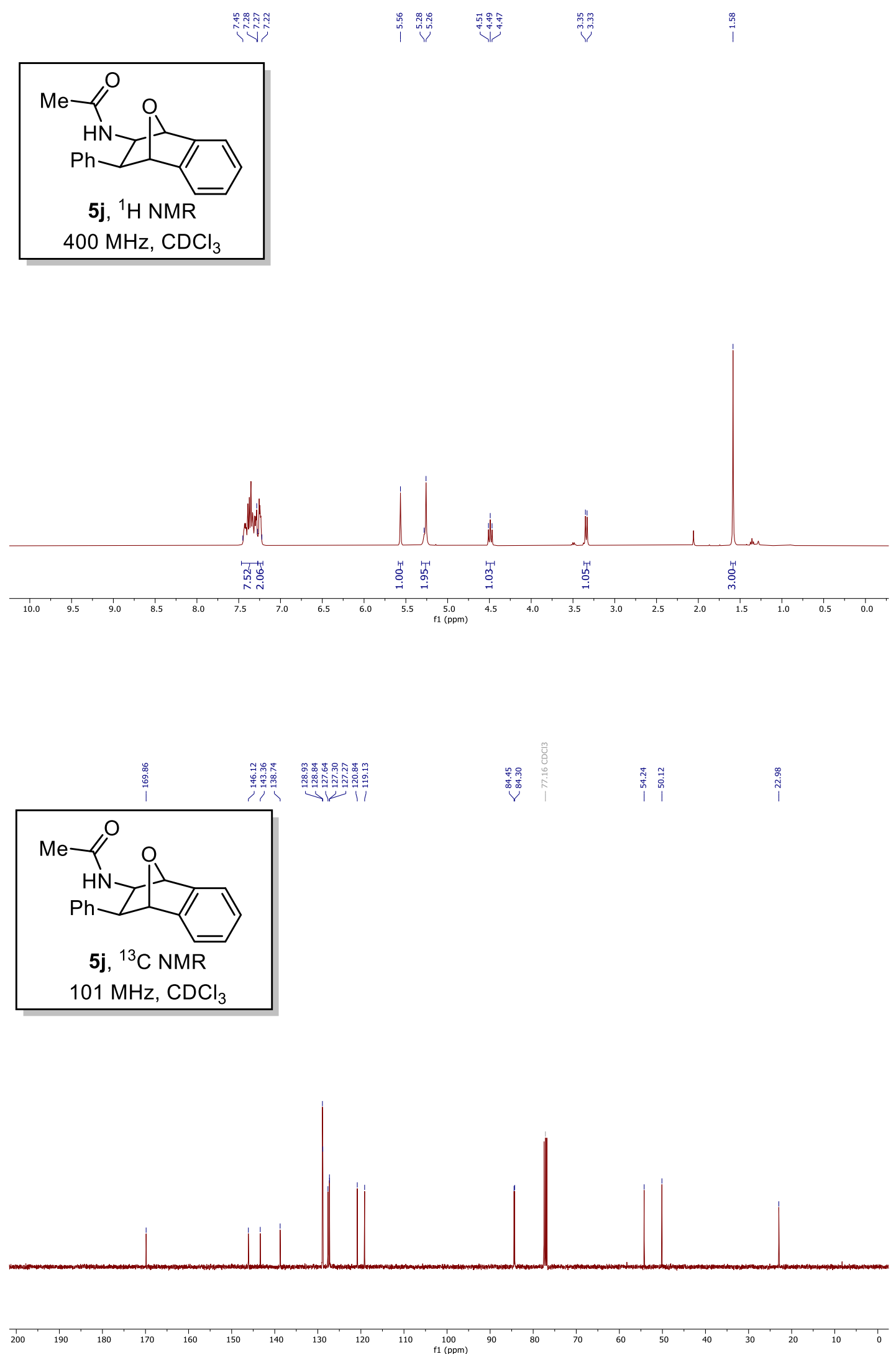

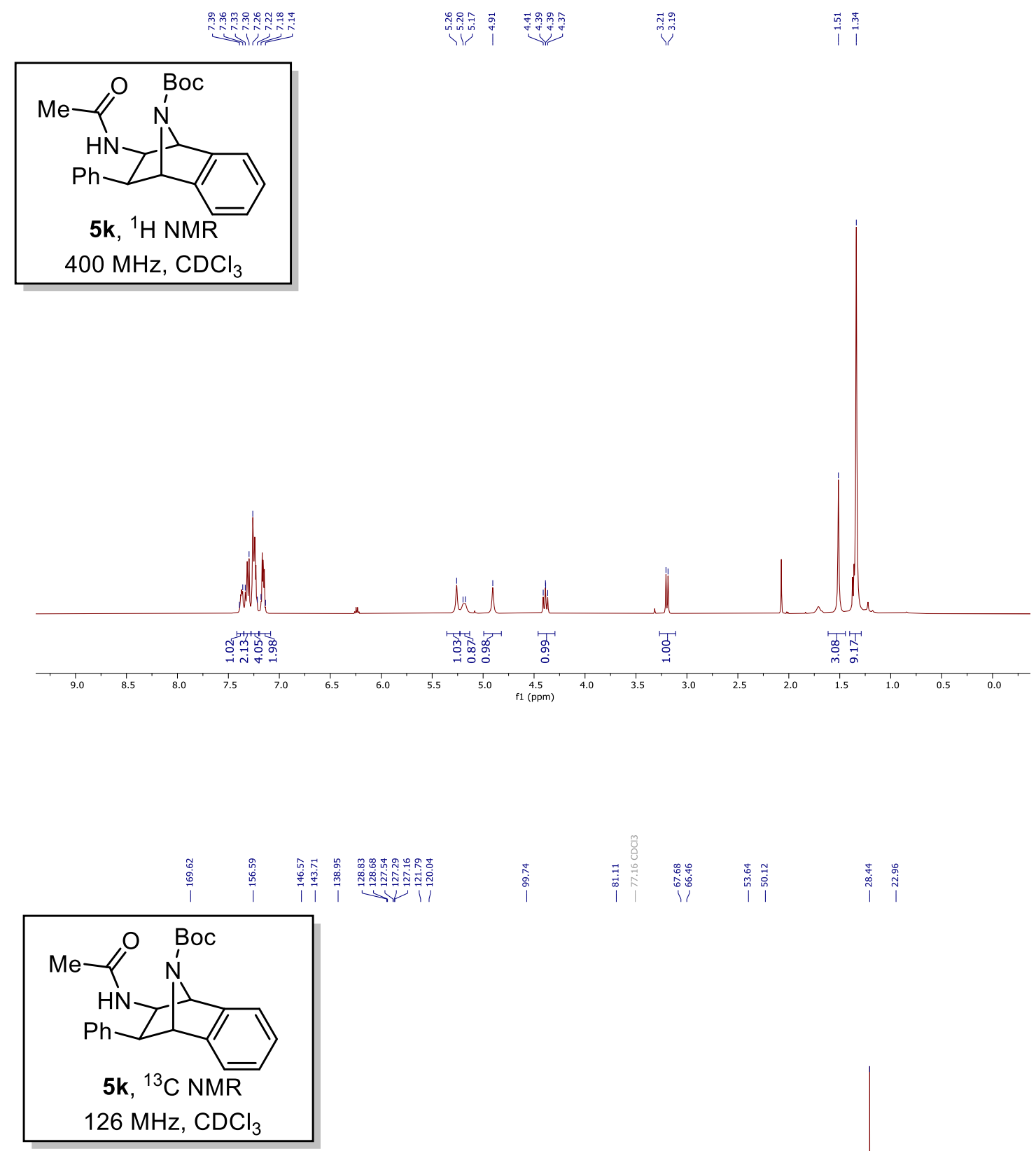

$\stackrel{\substack{* \\ \infty}}{\substack{\text { I } \\ \text { I }}}$

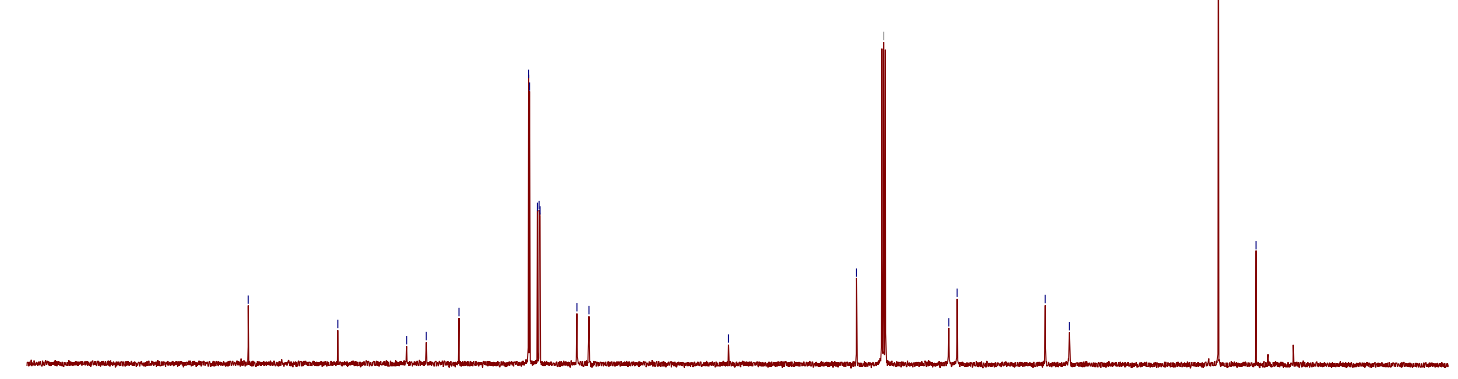

200
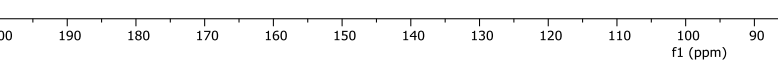

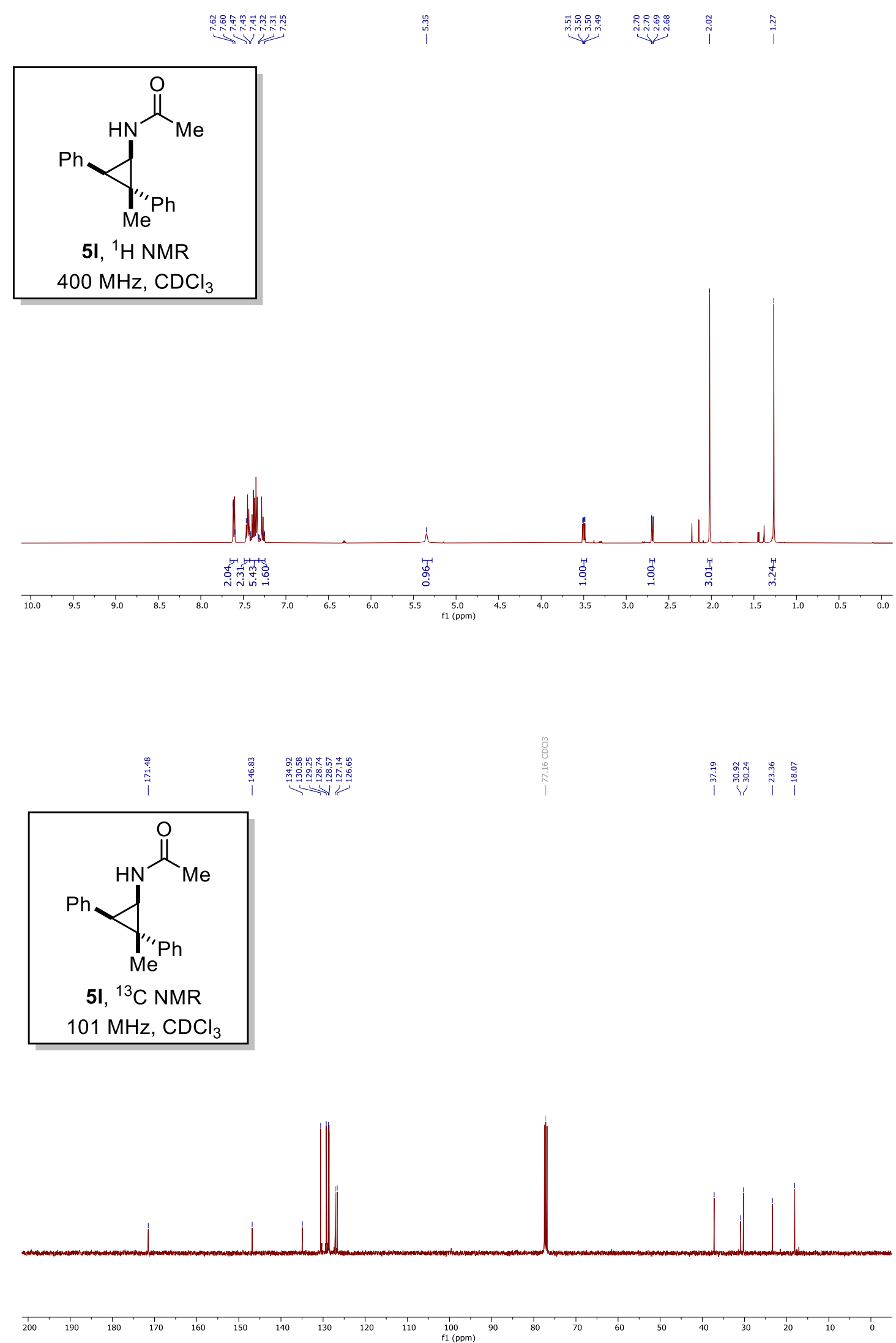


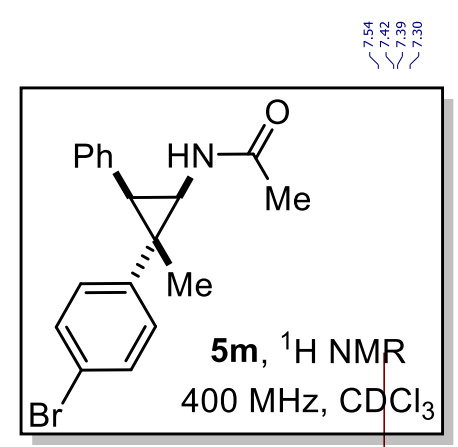

凩
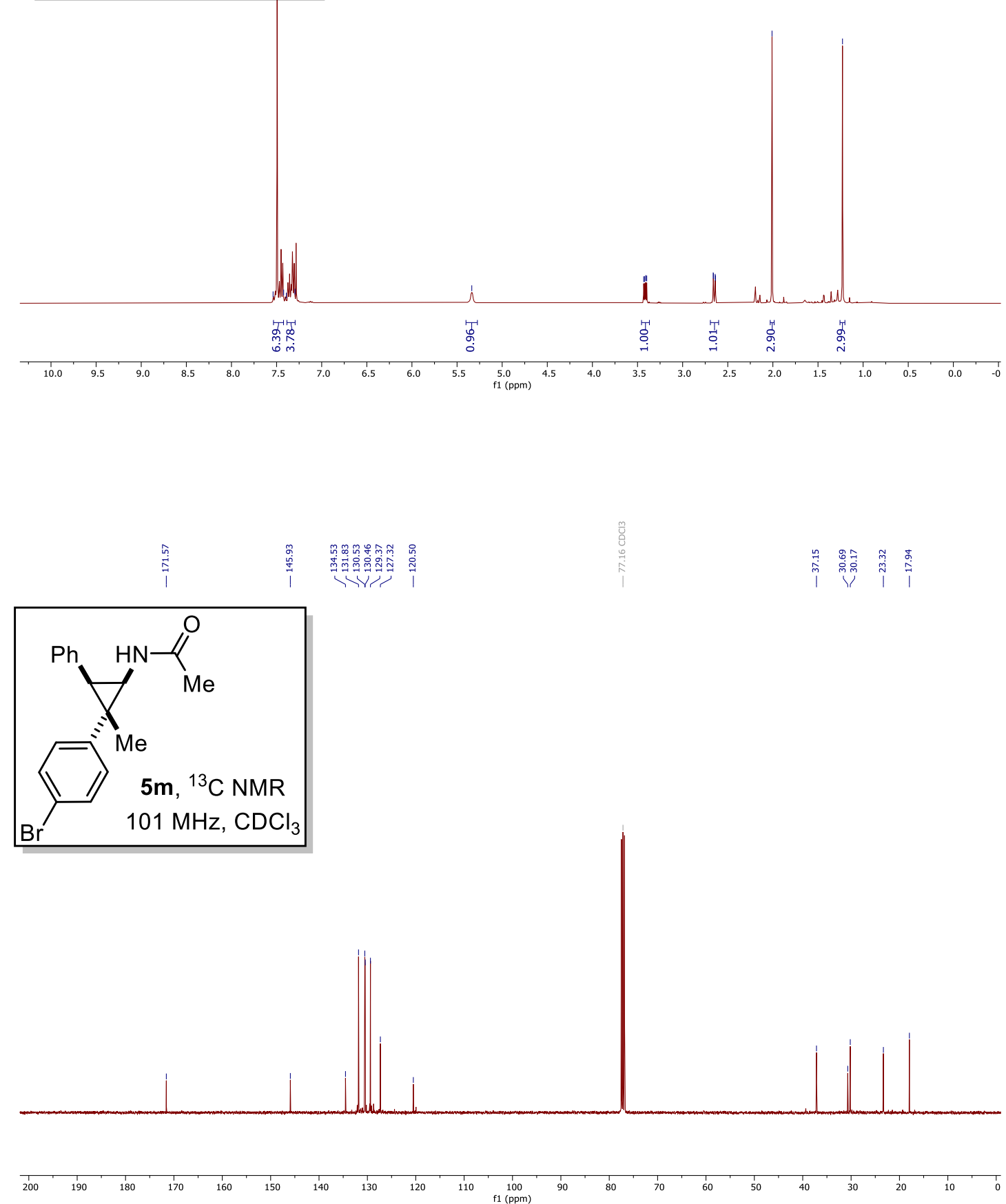

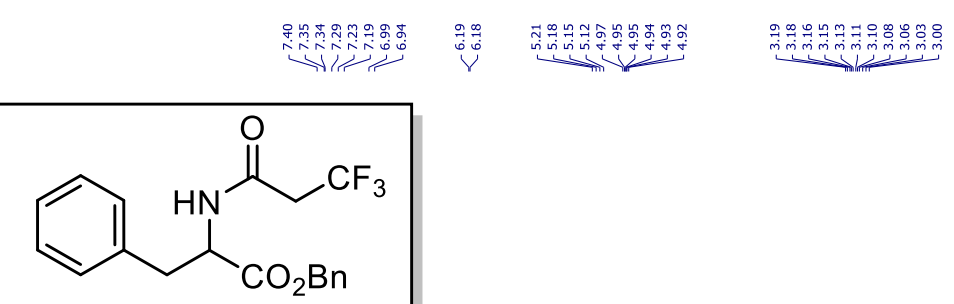

6b, ${ }^{1} \mathrm{H}$ NMR $400 \mathrm{MHz}, \mathrm{CDCl}_{3}$
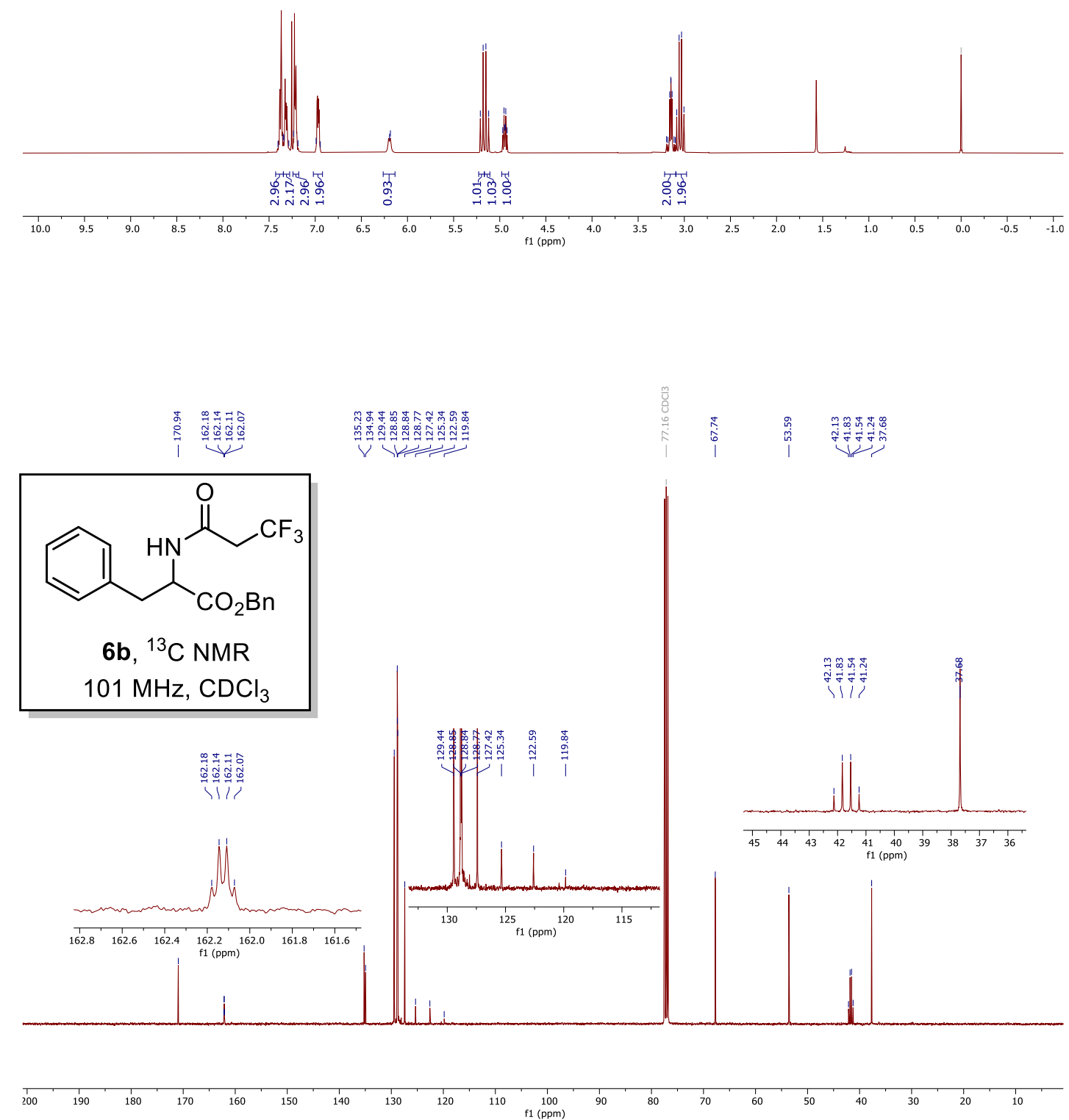


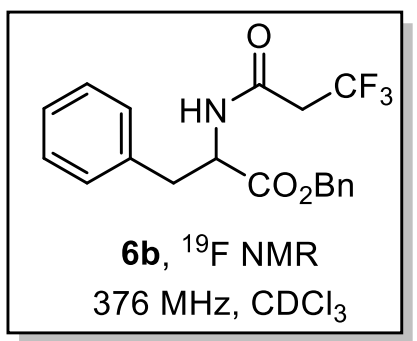

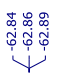

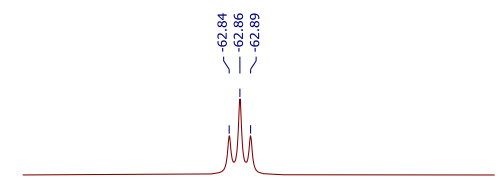

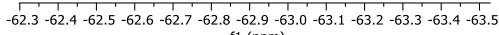

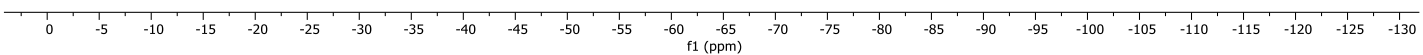



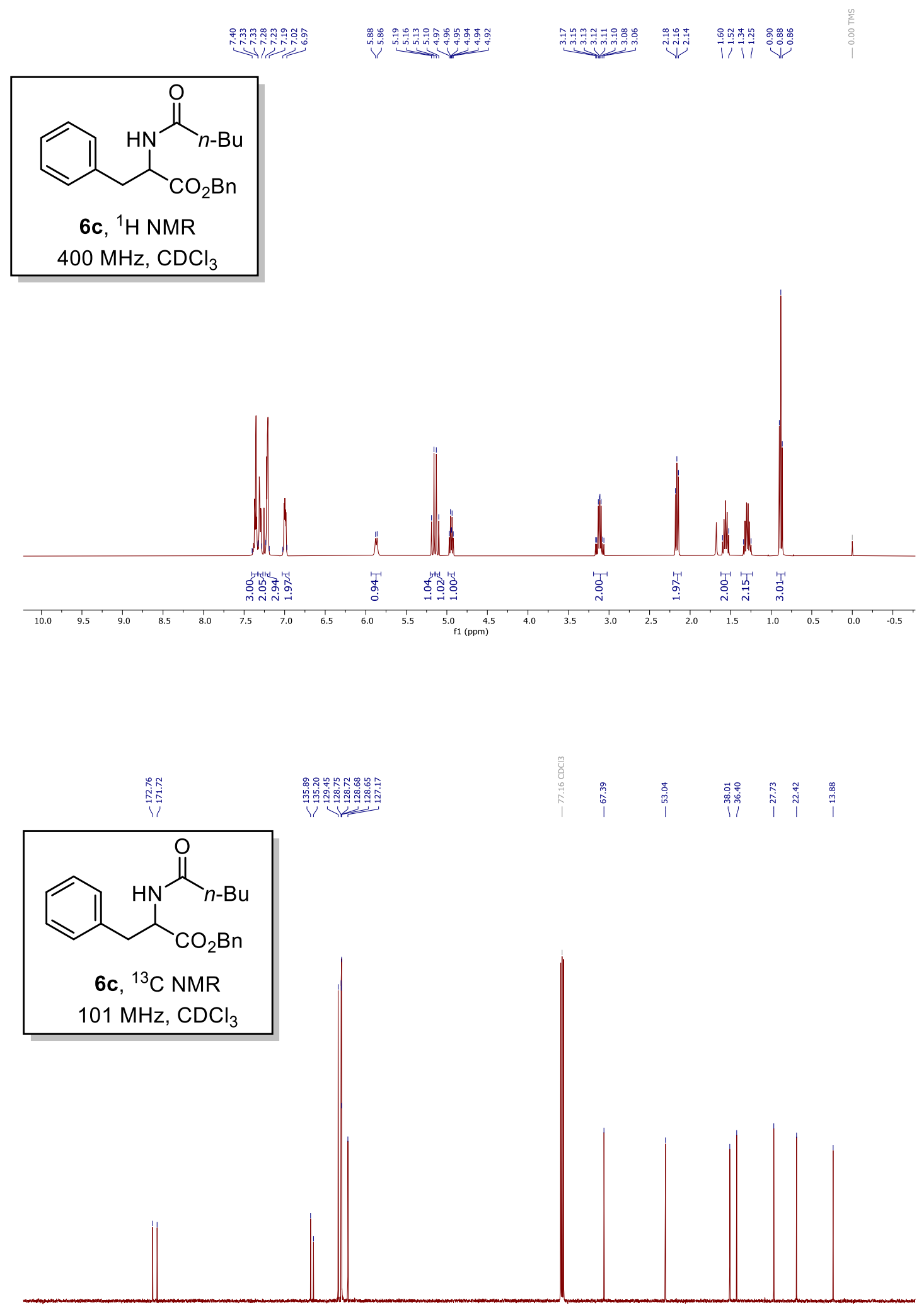

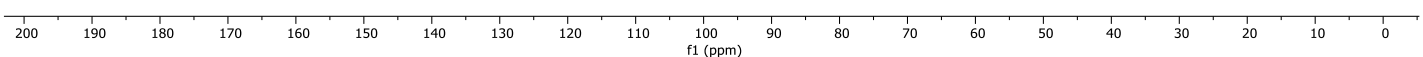



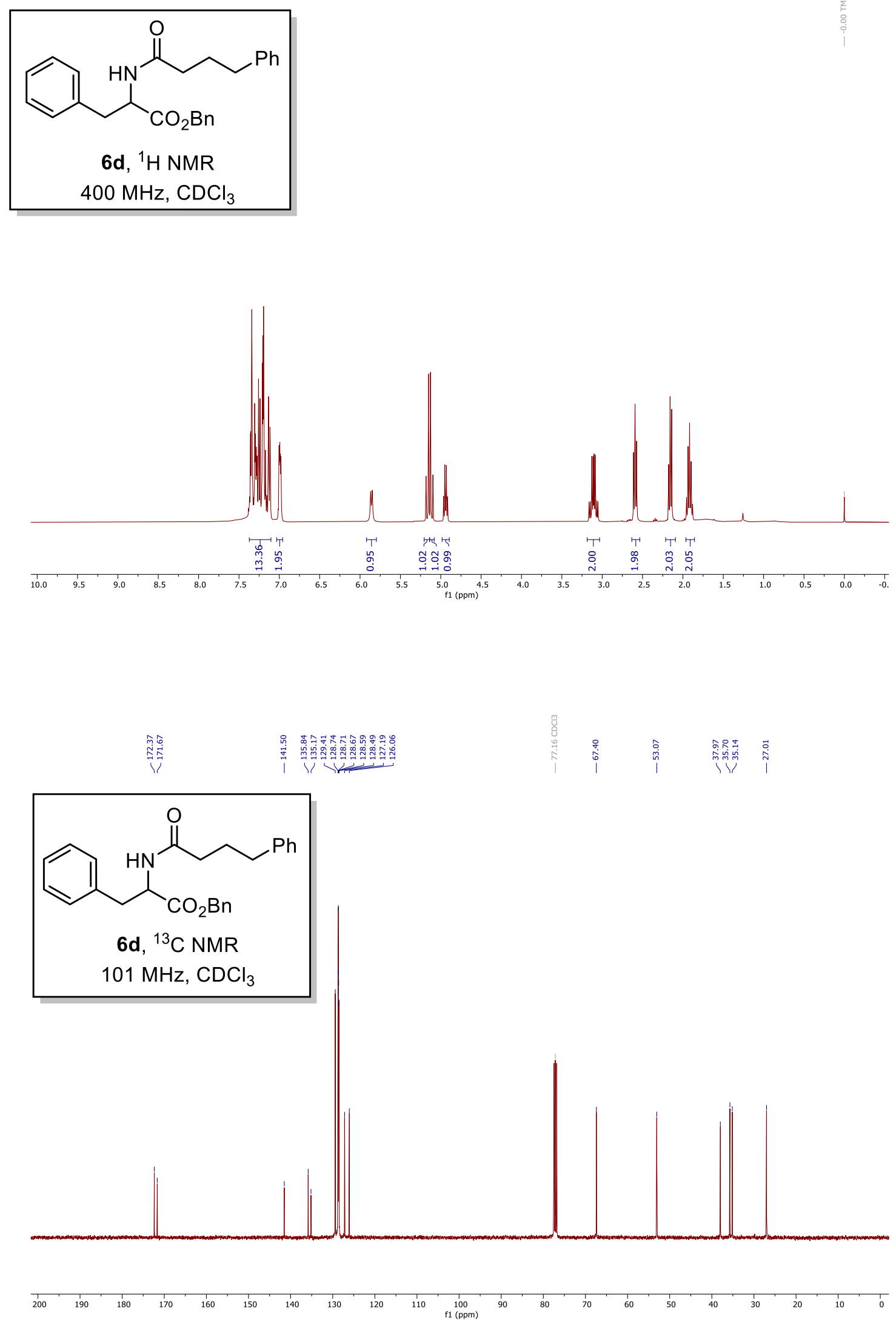

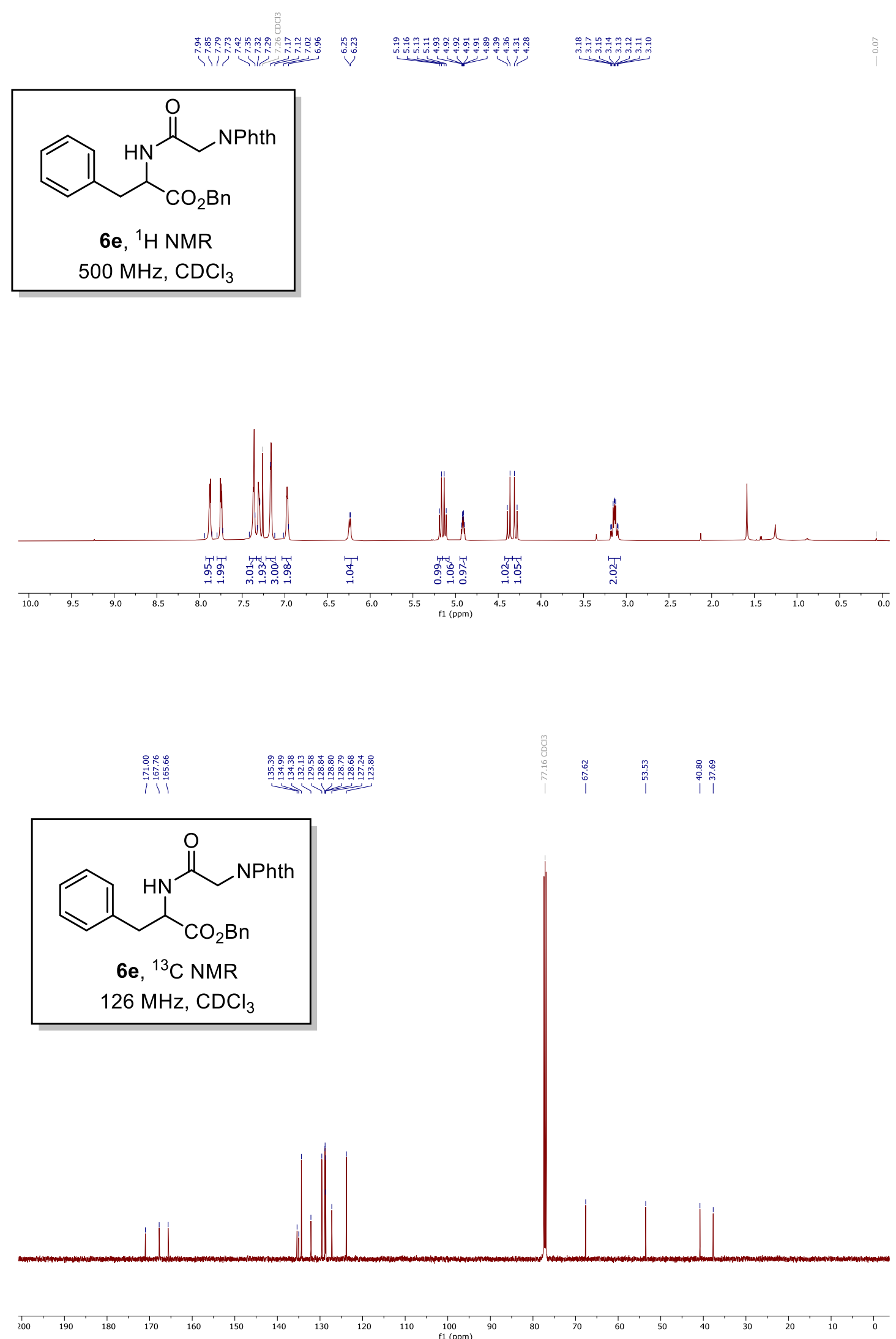

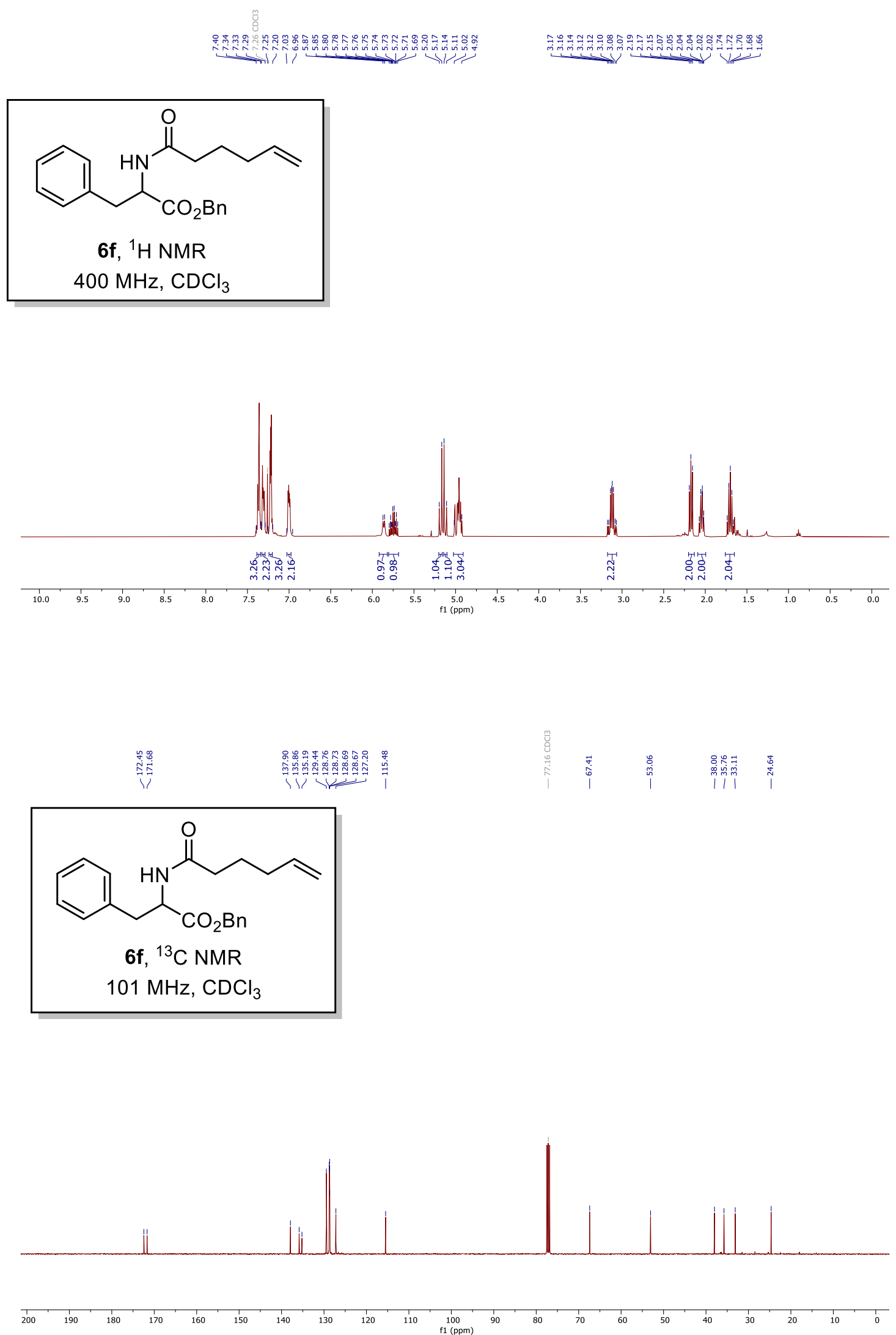
<smiles>O=C(NC(Cc1ccccc1)C(=O)OCc1ccccc1)C1CC1</smiles>

6g, ${ }^{1} \mathrm{H}$ NMR $400 \mathrm{MHz}, \mathrm{CDCl}_{3}$
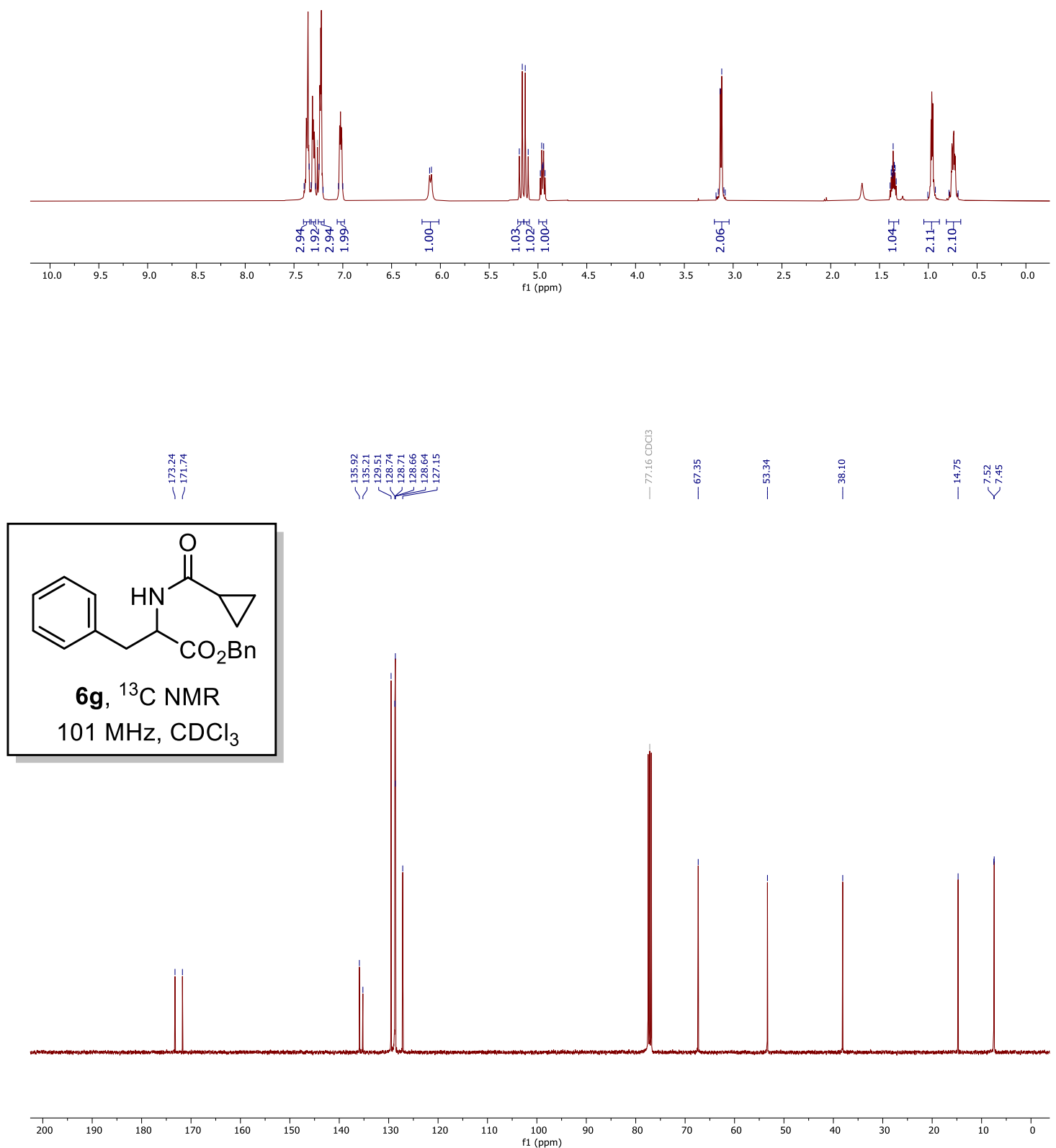

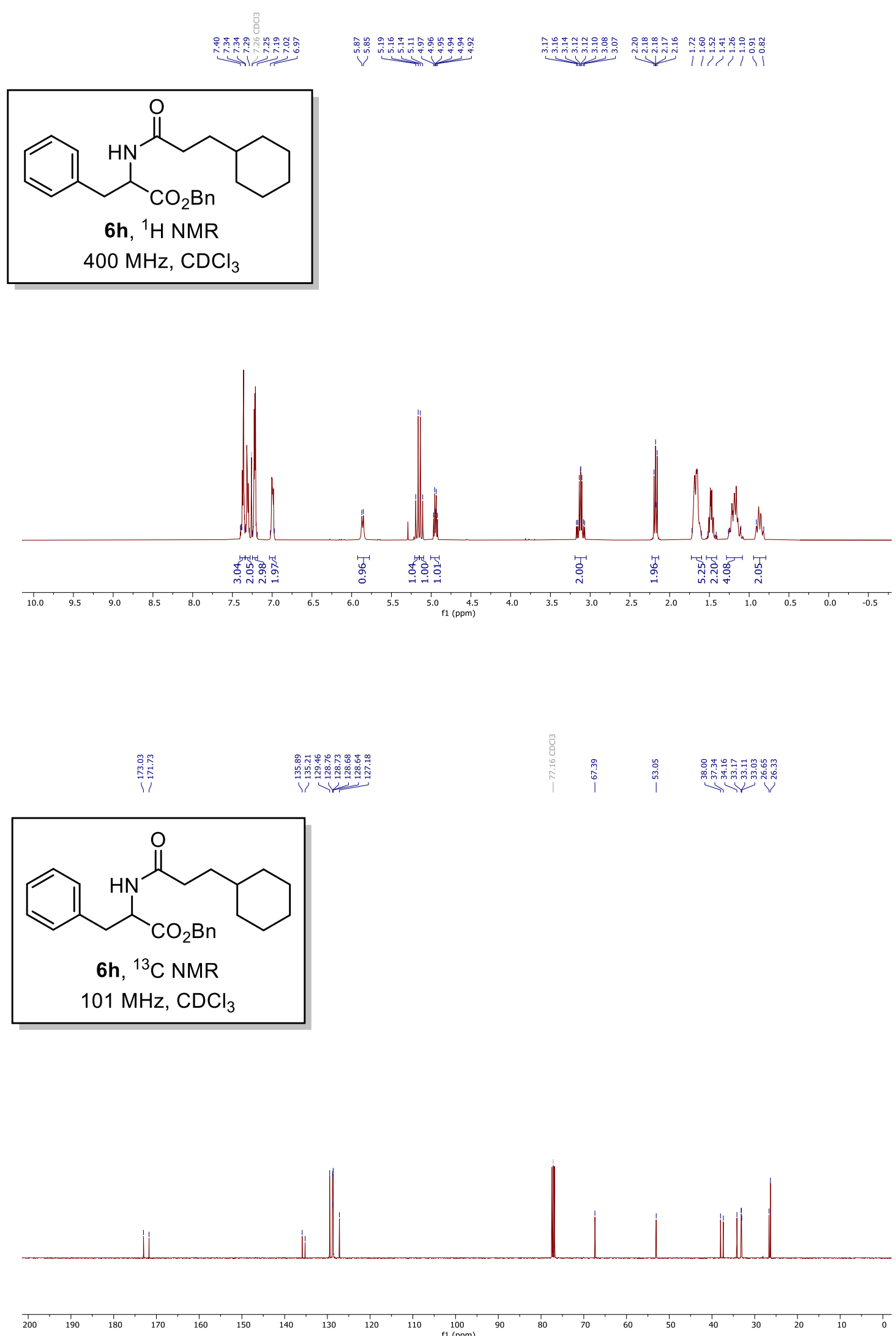

100
$\mathrm{f} 1(\mathrm{ppm})$ 
